\title{
Gallium(I)-Lanthanide(II) Donor-Acceptor bonds
}

\author{
Michal Wiecko and Peter W. Roesky* \\ Institut für Chemie, Freie Universität Berlin, Fabeckstraße 34-36, 14195 Berlin (Germany) \\ email: roesky@chemie.fu-berlin.de
}

Supporting Information 
Perspective ORTEP view of the molecular structure of $\mathbf{1}$. Thermal ellipsoids are drawn to encompass $50 \%$ probability. Hydrogen atoms are omitted for clarity.

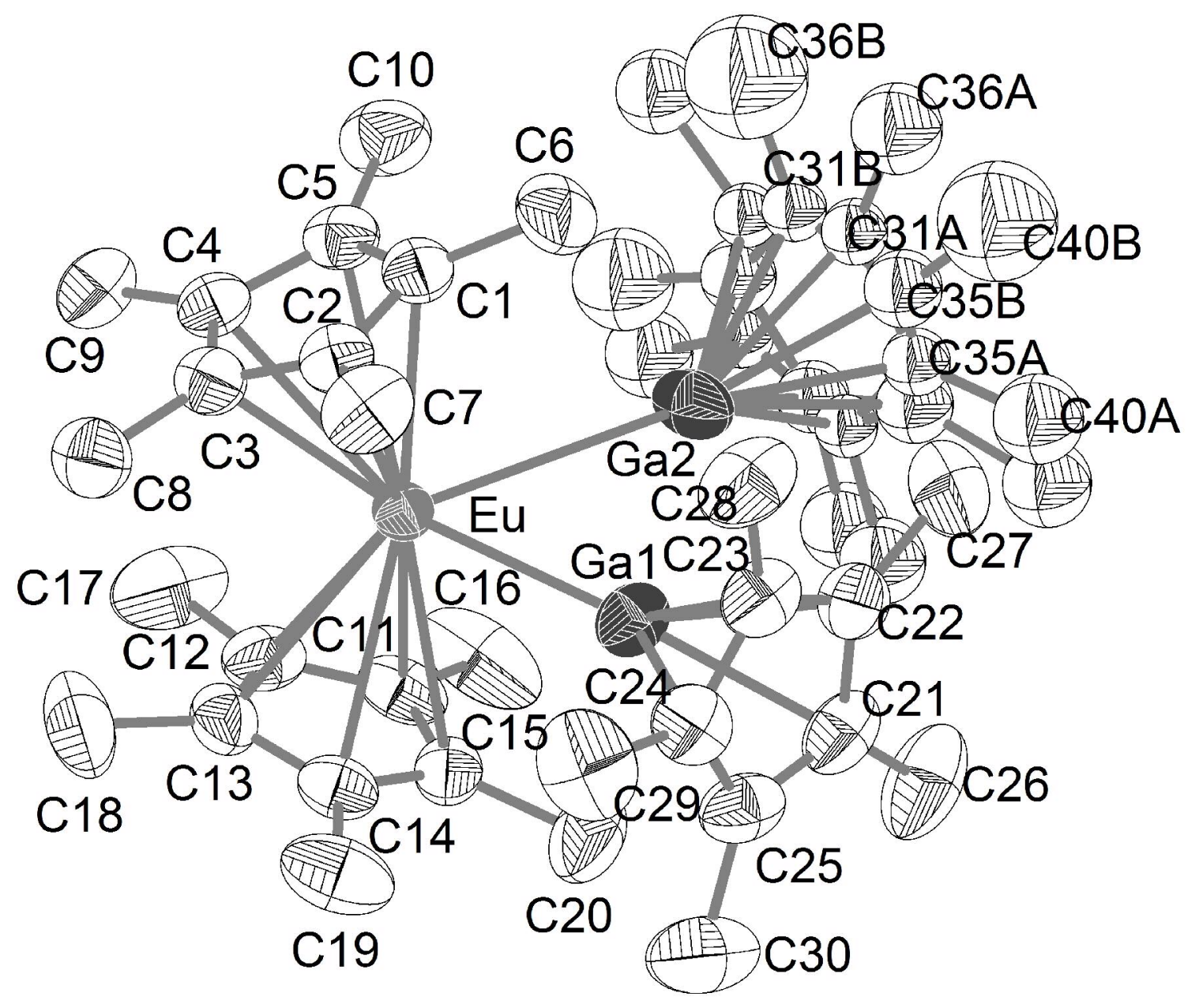


Table 1. Crystal data and structure refinement for 1.

\begin{tabular}{|c|c|}
\hline Identification code & $\mathrm{z}: \backslash$ daten \wiecko \mw33-cpseu (gacps) $2 \backslash \mathrm{mw} 3$ \\
\hline Empirical formula & C40 H60 Eu Ga2 \\
\hline Formula weight & 832.28 \\
\hline Temperature & $200(2) \mathrm{K}$ \\
\hline Wavelength & $0.71073 \mathrm{~A}$ \\
\hline Crystal system, space group & Monoclinic, $\quad$ C $2 / C$ \\
\hline Unit cell dimensions & $\begin{aligned} \mathrm{a}=20.0374(14) \mathrm{A} & \text { alpha }=90 \text { deg } . \\
\mathrm{b}=12.3041(16) \mathrm{A} & \text { beta }=93.294(6)\end{aligned}$ \\
\hline & $\mathrm{c}=32.450(2)$ A gamma $=90 \mathrm{deg}$. \\
\hline Volume & $7987.1(13) \quad A \wedge 3$ \\
\hline Z, Calculated density & $8, \quad 1.384 \mathrm{Mg} / \mathrm{m} \wedge 3$ \\
\hline Absorption coefficient & $2.914 \mathrm{~mm} \wedge-1$ \\
\hline$F(000)$ & 3400 \\
\hline Crystal size & $0.522 \times 0.498 \times 0.479 \mathrm{~mm}$ \\
\hline Theta range for data collection & 2.67 to $25.03 \mathrm{deg}$. \\
\hline Limiting indices & $-23<=\mathrm{h}<=23, \quad-14<=\mathrm{k}<=14, \quad-32<=1<=38$ \\
\hline Reflections collected / unique & $28190 / 7035[R($ int $)=0.0750]$ \\
\hline Completeness to theta $=25.03$ & $99.7 \%$ \\
\hline Absorption correction & Integration \\
\hline Max. and min. transmission & 0.7495 and 0.5340 \\
\hline Refinement method & Full-matrix least-squares on $F \wedge 2$ \\
\hline Data / restraints / parameters & $7035 / 0 / 413$ \\
\hline Goodness-of-fit on $F^{\wedge} 2$ & 1.034 \\
\hline Final R indices [I>2sigma(I)] & $\mathrm{R} 1=0.0385, \mathrm{wR} 2=0.0985$ \\
\hline R indices (all data) & $\mathrm{R} 1=0.0440, \mathrm{wR} 2=0.1014$ \\
\hline Largest diff. peak and hole & 0.805 and -1.182 e.A^-3 \\
\hline
\end{tabular}


Table 2. Atomic coordinates $(\mathrm{x} 10 \wedge 4)$ and equivalent isotropic displacement parameters $(A \wedge 2 \times 10 \wedge 3)$ for 1 .

$\mathrm{U}(\mathrm{eq})$ is defined as one third of the trace of the orthogonalized Uij tensor.

\begin{tabular}{|c|c|c|c|c|}
\hline & $x$ & y & $\mathrm{z}$ & $\mathrm{U}(\mathrm{eq})$ \\
\hline$C(1)$ & $2140(2)$ & $11(4)$ & $-347(1)$ & $49(1)$ \\
\hline$C(2)$ & $1493(2)$ & $473(4)$ & $-375(1)$ & $48(1)$ \\
\hline$C(3)$ & $1557(2)$ & $1588(4)$ & $-485(1)$ & $49(1)$ \\
\hline$C(4)$ & $2244(2)$ & $1811(4)$ & $-518(1)$ & $47(1)$ \\
\hline$C(5)$ & $2598(2)$ & $840(4)$ & $-433(1)$ & $49(1)$ \\
\hline$C(6)$ & $2307(3)$ & $-1138(4)$ & $-215(2)$ & $71(2)$ \\
\hline$C(7)$ & $850(3)$ & $-56(5)$ & $-265(2)$ & $70(1)$ \\
\hline$C(8)$ & $989(3)$ & $2382(5)$ & $-506(2)$ & $66(1)$ \\
\hline$C(9)$ & $2541(3)$ & $2915(4)$ & $-589(2)$ & $62(1)$ \\
\hline $\mathrm{C}(10)$ & $3349(3)$ & $722(5)$ & $-407(2)$ & $73(2)$ \\
\hline$C(11)$ & $2169(2)$ & $983(4)$ & $-2011(1)$ & $55(1)$ \\
\hline C (12) & $2053(3)$ & $1992(4)$ & $-1821(1)$ & $56(1)$ \\
\hline$C(13)$ & $1377(3)$ & $2043(4)$ & $-1731(1)$ & $52(1)$ \\
\hline $\mathrm{C}(14)$ & $1079(2)$ & $1070(4)$ & $-1863(1)$ & $49(1)$ \\
\hline$C(15)$ & $1558(3)$ & $412(3)$ & $-2037(1)$ & $50(1)$ \\
\hline $\mathrm{C}(16)$ & $2805(3)$ & $650(7)$ & $-2199(2)$ & $102(3)$ \\
\hline$C(17)$ & $2549(4)$ & $2916(6)$ & $-1777(2)$ & $99(3)$ \\
\hline $\mathrm{C}(18)$ & $1037(4)$ & $3039(5)$ & $-1578(2)$ & $100(2)$ \\
\hline $\mathrm{C}(19)$ & $337(3)$ & $813(6)$ & $-1845(2)$ & $84(2)$ \\
\hline$C(20)$ & $1447(4)$ & $-668(4)$ & $-2249(2)$ & $85(2)$ \\
\hline$C(21)$ & $769(3)$ & $-3348(4)$ & $-1531(2)$ & $61(1)$ \\
\hline C (22) & $177(3)$ & $-2732(4)$ & $-1535(2)$ & $60(1)$ \\
\hline$C(23)$ & $6(3)$ & $-2584(4)$ & $-1121(2)$ & $61(1)$ \\
\hline $\mathrm{C}(24)$ & $491(3)$ & $-3119(4)$ & $-862(2)$ & $58(1)$ \\
\hline$C(25)$ & $971(3)$ & $-3592(4)$ & $-1113(2)$ & $58(1)$ \\
\hline$C(26)$ & $1128(4)$ & $-3721(5)$ & $-1904(2)$ & $94(2)$ \\
\hline$C(27)$ & $-227(4)$ & $-2355(6)$ & $-1914(2)$ & $97(2)$ \\
\hline$C(28)$ & $-601(3)$ & $-2000(6)$ & $-979(3)$ & $97(2)$ \\
\hline C (29) & $484(4)$ & $-3243(5)$ & $-403(2)$ & $86(2)$ \\
\hline$C(30)$ & $1563(4)$ & $-4250(5)$ & $-963(3)$ & $94(2)$ \\
\hline$C(31 A)$ & $3969(2)$ & $-2579(3)$ & $-1108(1)$ & $45(1)$ \\
\hline$C(32 A)$ & $4268(2)$ & $-1550(3)$ & $-1181(1)$ & $42(1)$ \\
\hline$C(33 A)$ & $4181(2)$ & $-1320(3)$ & $-1611(1)$ & $42(1)$ \\
\hline$C(34 A)$ & $3829(2)$ & $-2207(4)$ & $-1804(1)$ & $40(1)$ \\
\hline$C(35 A)$ & $3698(2)$ & $-2986(3)$ & $-1494(1)$ & $46(1)$ \\
\hline$C(36 A)$ & $3945(3)$ & $-3143(6)$ & $-694(2)$ & $84(2)$ \\
\hline$C(37 A)$ & $4653(4)$ & $-846(7)$ & $-874(3)$ & $72(2)$ \\
\hline$C(38 A)$ & $4476(5)$ & $-356(7)$ & $-1818(3)$ & $78(2)$ \\
\hline$C(39 A)$ & $3673(5)$ & $-2330(8)$ & $-2263(3)$ & $75(2)$ \\
\hline$C(40 A)$ & $3383(5)$ & $-4057(8)$ & $-1573(3)$ & $82(2)$ \\
\hline$C(31 B)$ & $4063(6)$ & $-2075(10)$ & $-1060(3)$ & $42(3)$ \\
\hline$C(32 B)$ & $4210(7)$ & $-1398(9)$ & $-1390(4)$ & $57(4)$ \\
\hline$C(33 B)$ & $3975(7)$ & $-1906(11)$ & $-1757(4)$ & $53(4)$ \\
\hline$C(34 B)$ & $3683(7)$ & $-2896(11)$ & $-1654(4)$ & $60(5)$ \\
\hline$C(35 B)$ & $3737(7)$ & $-3000(10)$ & $-1223(4)$ & $61(4)$ \\
\hline$C(36 B)$ & $4224(11)$ & $-1850(19)$ & $-615(6)$ & $155(14)$ \\
\hline$C(37 B)$ & $4489(16)$ & $-290(20)$ & $-1531(10)$ & $106(9)$ \\
\hline
\end{tabular}




\begin{tabular}{lllcr}
$\mathrm{C}(38 \mathrm{~B})$ & $3928(12)$ & $-1940(20)$ & $-2246(7)$ & $77(6)$ \\
$\mathrm{C}(39 \mathrm{~B})$ & $3381(13)$ & $-4002(19)$ & $-1826(8)$ & $80(6)$ \\
$\mathrm{C}(40 \mathrm{~B})$ & $3580(20)$ & $-4000(30)$ & $-888(13)$ & $146(13)$ \\
$\mathrm{Ga}(1)$ & $1024(1)$ & $-1712(1)$ & $-1196(1)$ & $55(1)$ \\
$\mathrm{Ga}(2)$ & $3103(1)$ & $-1419(1)$ & $-1357(1)$ & $62(1)$ \\
$\mathrm{Eu}$ & $1926(1)$ & $484(1)$ & $-1188(1)$ & $39(1)$ \\
\hline
\end{tabular}


Table 3. Bond lengths [A] and angles [deg] for 1.

\begin{tabular}{|c|c|}
\hline$C(1)-C(5)$ & $1.411(7)$ \\
\hline$C(1)-C(2)$ & $1.414(6)$ \\
\hline$C(1)-C(6)$ & $1.508(7)$ \\
\hline$C(1)-E u$ & $2.800(4)$ \\
\hline$C(2)-C(3)$ & $1.426(6)$ \\
\hline$C(2)-C(7)$ & $1.505(7)$ \\
\hline$C(2)-E u$ & $2.825(4)$ \\
\hline$C(3)-C(4)$ & $1.414(6)$ \\
\hline$C(3)-C(8)$ & $1.497(7)$ \\
\hline$C(3)-E u$ & $2.792(4)$ \\
\hline$C(4)-C(5)$ & $1.409(6)$ \\
\hline$C(4)-C(9)$ & $1.506(7)$ \\
\hline$C(4)-E u$ & $2.763(4)$ \\
\hline$C(5)-C(10)$ & $1.508(7)$ \\
\hline$C(5)-E u$ & $2.763(4)$ \\
\hline$C(11)-C(15)$ & $1.410(7)$ \\
\hline$C(11)-C(12)$ & $1.412(7)$ \\
\hline$C(11)-C(16)$ & $1.503(7)$ \\
\hline$C(11)-E u$ & $2.810(4)$ \\
\hline$C(12)-C(13)$ & $1.403(7)$ \\
\hline$C(12)-C(17)$ & $1.512(7)$ \\
\hline$C(12)-E u$ & $2.789(4)$ \\
\hline$C(13)-C(14)$ & $1.394(7)$ \\
\hline$C(13)-C(18)$ & $1.500(7)$ \\
\hline$C(13)-E u$ & $2.786(4)$ \\
\hline$C(14)-C(15)$ & $1.398(7)$ \\
\hline$C(14)-C(19)$ & $1.524(7)$ \\
\hline$C(14)-E u$ & $2.787(4)$ \\
\hline$C(15)-C(20)$ & $1.507(7)$ \\
\hline$C(15)-E u$ & $2.811(4)$ \\
\hline$C(21)-C(22)$ & $1.407(8)$ \\
\hline$C(21)-C(25)$ & $1.425(7)$ \\
\hline$C(21)-C(26)$ & $1.514(8)$ \\
\hline $\mathrm{C}(21)-\mathrm{Ga}(1)$ & $2.330(5)$ \\
\hline$C(22)-C(23)$ & $1.418(8)$ \\
\hline$C(22)-C(27)$ & $1.506(8)$ \\
\hline $\mathrm{C}(22)-\mathrm{Ga}(1)$ & $2.335(5)$ \\
\hline$C(23)-C(24)$ & $1.411(7)$ \\
\hline$C(23)-C(28)$ & $1.508(8)$ \\
\hline $\mathrm{C}(23)-\mathrm{Ga}(1)$ & $2.330(5)$ \\
\hline$C(24)-C(25)$ & $1.420(8)$ \\
\hline$C(24)-C(29)$ & $1.498(7)$ \\
\hline $\mathrm{C}(24)-\mathrm{Ga}(1)$ & $2.334(5)$ \\
\hline$C(25)-C(30)$ & $1.495(8)$ \\
\hline $\mathrm{C}(25)-\mathrm{Ga}(1)$ & $2.332(5)$ \\
\hline$C(31 A)-C(35 A)$ & $1.426(3)$ \\
\hline$C(31 A)-C(32 A)$ & $1.426(3)$ \\
\hline$C(31 A)-C(36 A)$ & $1.516(3)$ \\
\hline$C(31 A)-G a(2)$ & $2.354(4)$ \\
\hline$C(32 A)-C(33 A)$ & $1.426(3)$ \\
\hline$C(32 A)-C(37 A)$ & $1.499(9)$ \\
\hline$C(32 A)-G a(2)$ & $2.376(4)$ \\
\hline$C(33 A)-C(34 A)$ & $1.426(3)$ \\
\hline$C(33 A)-C(38 A)$ & $1.501(10)$ \\
\hline
\end{tabular}




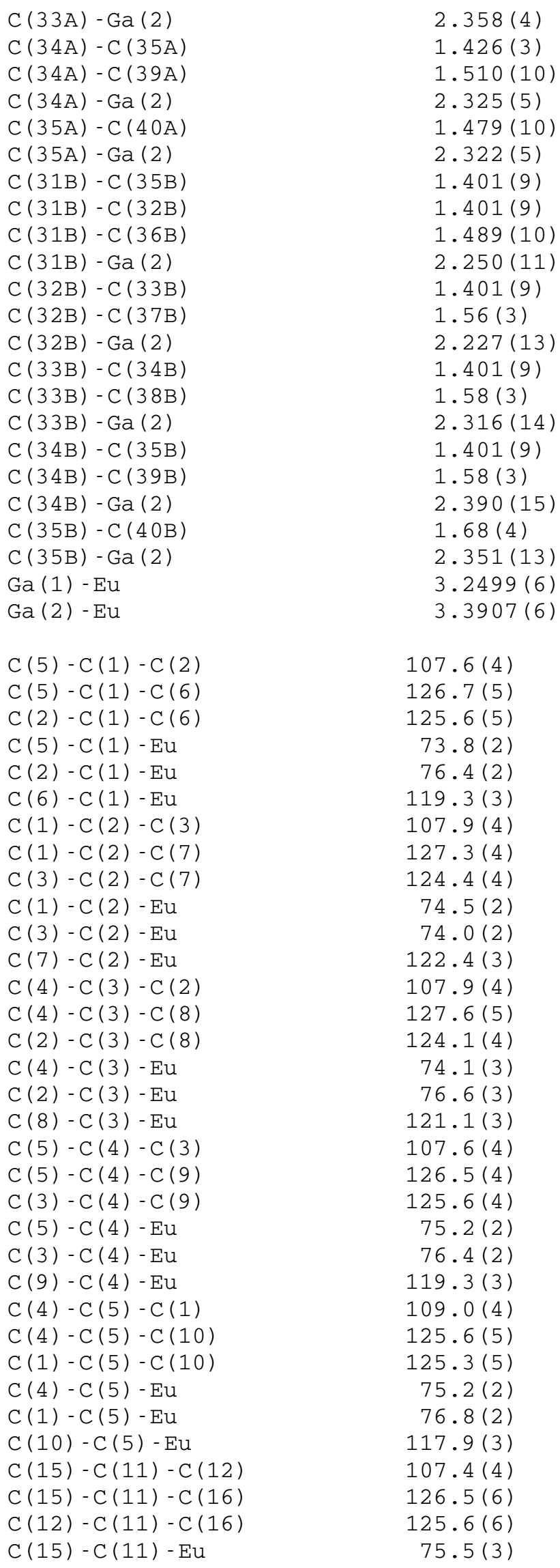




\begin{tabular}{|c|c|}
\hline$C(12)-C(11)-E u$ & $74.6(3)$ \\
\hline$C(16)-C(11)-E u$ & $121.7(3)$ \\
\hline$C(13)-C(12)-C(11)$ & $108.4(4)$ \\
\hline$C(13)-C(12)-C(17)$ & $125.7(6)$ \\
\hline$C(11)-C(12)-C(17)$ & $125.4(6)$ \\
\hline$C(13)-C(12)-E u$ & $75.3(3)$ \\
\hline$C(11)-C(12)-E u$ & $76.2(3)$ \\
\hline$C(17)-C(12)-E u$ & $121.3(3)$ \\
\hline$C(14)-C(13)-C(12)$ & $107.4(4)$ \\
\hline$C(14)-C(13)-C(18)$ & $127.5(5)$ \\
\hline$C(12)-C(13)-C(18)$ & $124.6(5)$ \\
\hline$C(14)-C(13)-E u$ & $75.5(2)$ \\
\hline$C(12)-C(13)-E u$ & $75.5(3)$ \\
\hline$C(18)-C(13)-E u$ & $121.6(3)$ \\
\hline$C(13)-C(14)-C(15)$ & $109.2(4)$ \\
\hline$C(13)-C(14)-C(19)$ & $124.6(5)$ \\
\hline$C(15)-C(14)-C(19)$ & $126.0(5)$ \\
\hline$C(13)-C(14)-E u$ & $75.5(2)$ \\
\hline$C(15)-C(14)-E u$ & $76.5(3)$ \\
\hline$C(19)-C(14)-E u$ & $117.9(3)$ \\
\hline$C(14)-C(15)-C(11)$ & $107.6(4)$ \\
\hline$C(14)-C(15)-C(20)$ & $127.5(5)$ \\
\hline$C(11)-C(15)-C(20)$ & $124.7(5)$ \\
\hline$C(14)-C(15)-E u$ & $74.6(3)$ \\
\hline$C(11)-C(15)-E u$ & $75.4(2)$ \\
\hline$C(20)-C(15)-E u$ & $119.9(3)$ \\
\hline$C(22)-C(21)-C(25)$ & $108.3(5)$ \\
\hline$C(22)-C(21)-C(26)$ & $126.5(5)$ \\
\hline$C(25)-C(21)-C(26)$ & $125.1(6)$ \\
\hline $\mathrm{C}(22)-\mathrm{C}(21)-\mathrm{Ga}(1)$ & $72.6(3)$ \\
\hline $\mathrm{C}(25)-\mathrm{C}(21)-\mathrm{Ga}(1)$ & $72.3(3)$ \\
\hline $\mathrm{C}(26)-\mathrm{C}(21)-\mathrm{Ga}(1)$ & $122.2(4)$ \\
\hline$C(21)-C(22)-C(23)$ & $108.1(4)$ \\
\hline$C(21)-C(22)-C(27)$ & $126.0(6)$ \\
\hline$C(23)-C(22)-C(27)$ & $125.9(6)$ \\
\hline $\mathrm{C}(21)-\mathrm{C}(22)-\mathrm{Ga}(1)$ & $72.3(3)$ \\
\hline $\mathrm{C}(23)-\mathrm{C}(22)-\mathrm{Ga}(1)$ & $72.1(3)$ \\
\hline $\mathrm{C}(27)-\mathrm{C}(22)-\mathrm{Ga}(1)$ & $124.1(4)$ \\
\hline$C(24)-C(23)-C(22)$ & $108.0(5)$ \\
\hline$C(24)-C(23)-C(28)$ & $125.5(6)$ \\
\hline$C(22)-C(23)-C(28)$ & $126.5(5)$ \\
\hline $\mathrm{C}(24)-\mathrm{C}(23)-\mathrm{Ga}(1)$ & $72.6(3)$ \\
\hline $\mathrm{C}(22)-\mathrm{C}(23)-\mathrm{Ga}(1)$ & $72.5(3)$ \\
\hline $\mathrm{C}(28)-\mathrm{C}(23)-\mathrm{Ga}(1)$ & $122.6(4)$ \\
\hline$C(23)-C(24)-C(25)$ & $108.3(4)$ \\
\hline$C(23)-C(24)-C(29)$ & $126.5(6)$ \\
\hline$C(25)-C(24)-C(29)$ & $125.1(5)$ \\
\hline $\mathrm{C}(23)-\mathrm{C}(24)-\mathrm{Ga}(1)$ & $72.2(3)$ \\
\hline $\mathrm{C}(25)-\mathrm{C}(24)-\mathrm{Ga}(1)$ & $72.2(3)$ \\
\hline $\mathrm{C}(29)-\mathrm{C}(24)-\mathrm{Ga}(1)$ & $124.6(4)$ \\
\hline$C(24)-C(25)-C(21)$ & $107.2(5)$ \\
\hline$C(24)-C(25)-C(30)$ & $125.9(5)$ \\
\hline$C(21)-C(25)-C(30)$ & $126.9(6)$ \\
\hline $\mathrm{C}(24)-\mathrm{C}(25)-\mathrm{Ga}(1)$ & $72.4(3)$ \\
\hline $\mathrm{C}(21)-\mathrm{C}(25)-\mathrm{Ga}(1)$ & $72.1(3)$ \\
\hline $\mathrm{C}(30)-\mathrm{C}(25)-\mathrm{Ga}(1)$ & $122.3(4)$ \\
\hline$C(35 A)-C(31 A)-C(32 A)$ & 108.0 \\
\hline$C(35 A)-C(31 A)-C(36 A)$ & 126.0 \\
\hline
\end{tabular}




\begin{tabular}{|c|c|}
\hline$C(32 A)-C(31 A)-C(36 A)$ & 126.0 \\
\hline$C(35 A)-C(31 A)-G a(2)$ & $71.04(16)$ \\
\hline$C(32 A)-C(31 A)-G a(2)$ & $73.30(16)$ \\
\hline$C(36 A)-C(31 A)-G a(2)$ & $121.39(13)$ \\
\hline$C(33 A)-C(32 A)-C(31 A)$ & 108.0 \\
\hline$C(33 A)-C(32 A)-C(37 A)$ & $124.4(4)$ \\
\hline$C(31 A)-C(32 A)-C(37 A)$ & $127.5(4)$ \\
\hline$C(33 A)-C(32 A)-G a(2)$ & $71.79(13)$ \\
\hline$C(31 A)-C(32 A)-G a(2)$ & $71.62(15)$ \\
\hline$C(37 A)-C(32 A)-G a(2)$ & $125.5(4)$ \\
\hline$C(32 A)-C(33 A)-C(34 A)$ & 108.0 \\
\hline$C(32 A)-C(33 A)-C(38 A)$ & $124.5(5)$ \\
\hline$C(34 A)-C(33 A)-C(38 A)$ & $127.3(5)$ \\
\hline$C(32 A)-C(33 A)-G a(2)$ & $73.16(14)$ \\
\hline$C(34 A)-C(33 A)-G a(2)$ & $71.00(17)$ \\
\hline$C(38 A)-C(33 A)-G a(2)$ & $126.1(5)$ \\
\hline$C(35 A)-C(34 A)-C(33 A)$ & 108.0 \\
\hline$C(35 A)-C(34 A)-C(39 A)$ & $126.5(5)$ \\
\hline$C(33 A)-C(34 A)-C(39 A)$ & $125.4(5)$ \\
\hline$C(35 A)-C(34 A)-G a(2)$ & $72.03(14)$ \\
\hline$C(33 A)-C(34 A)-G a(2)$ & $73.56(17)$ \\
\hline$C(39 A)-C(34 A)-G a(2)$ & $123.8(4)$ \\
\hline$C(31 A)-C(35 A)-C(34 A)$ & 108.0 \\
\hline$C(31 A)-C(35 A)-C(40 A)$ & $127.1(5)$ \\
\hline$C(34 A)-C(35 A)-C(40 A)$ & $124.7(5)$ \\
\hline$C(31 A)-C(35 A)-G a(2)$ & $73.47(15)$ \\
\hline$C(34 A)-C(35 A)-G a(2)$ & $72.23(14)$ \\
\hline$C(40 A)-C(35 A)-G a(2)$ & $123.7(5)$ \\
\hline$C(35 B)-C(31 B)-C(32 B)$ & 108.0 \\
\hline$C(35 B)-C(31 B)-C(36 B)$ & 126.0 \\
\hline$C(32 B)-C(31 B)-C(36 B)$ & 126.0 \\
\hline$C(35 B)-C(31 B)-G a(2)$ & $76.2(5)$ \\
\hline$C(32 B)-C(31 B)-G a(2)$ & $70.9(5)$ \\
\hline$C(36 B)-C(31 B)-G a(2)$ & $118.7(4)$ \\
\hline$C(33 B)-C(32 B)-C(31 B)$ & 108.0 \\
\hline$C(33 B)-C(32 B)-C(37 B)$ & $104.6(15)$ \\
\hline$C(31 B)-C(32 B)-C(37 B)$ & $147.3(15)$ \\
\hline$C(33 B)-C(32 B)-G a(2)$ & $75.5(5)$ \\
\hline$C(31 B)-C(32 B)-G a(2)$ & $72.6(5)$ \\
\hline$C(37 B)-C(32 B)-G a(2)$ & $113.5(14)$ \\
\hline$C(34 B)-C(33 B)-C(32 B)$ & 108.0 \\
\hline$C(34 B)-C(33 B)-C(38 B)$ & $102.6(13)$ \\
\hline$C(32 B)-C(33 B)-C(38 B)$ & $149.2(13)$ \\
\hline$C(34 B)-C(33 B)-G a(2)$ & $75.6(5)$ \\
\hline$C(32 B)-C(33 B)-G a(2)$ & $68.6(5)$ \\
\hline$C(38 B)-C(33 B)-G a(2)$ & $124.3(12)$ \\
\hline$C(33 B)-C(34 B)-C(35 B)$ & 108.0 \\
\hline$C(33 B)-C(34 B)-C(39 B)$ & $145.3(13)$ \\
\hline$C(35 B)-C(34 B)-C(39 B)$ & $106.3(13)$ \\
\hline$C(33 B)-C(34 B)-G a(2)$ & $69.8(5)$ \\
\hline$C(35 B)-C(34 B)-G a(2)$ & $71.3(4)$ \\
\hline$C(39 B)-C(34 B)-G a(2)$ & $127.8(12)$ \\
\hline$C(34 B)-C(35 B)-C(31 B)$ & 108.0 \\
\hline$C(34 B)-C(35 B)-C(40 B)$ & $134.8(16)$ \\
\hline$C(31 B)-C(35 B)-C(40 B)$ & $116.8(16)$ \\
\hline$C(34 B)-C(35 B)-G a(2)$ & $74.4(4)$ \\
\hline$C(31 B)-C(35 B)-G a(2)$ & $68.4(5)$ \\
\hline$C(40 B)-C(35 B)-G a(2)$ & $127.2(16)$ \\
\hline
\end{tabular}




\begin{tabular}{|c|c|}
\hline $\mathrm{C}(23)-\mathrm{Ga}(1)-\mathrm{C}(21)$ & $58.76(19)$ \\
\hline $\mathrm{C}(23)-\mathrm{Ga}(1)-\mathrm{C}(25)$ & $58.98(19)$ \\
\hline $\mathrm{C}(21)-\mathrm{Ga}(1)-\mathrm{C}(25)$ & $35.59(18)$ \\
\hline $\mathrm{C}(23)-\mathrm{Ga}(1)-\mathrm{C}(24)$ & $35.22(18)$ \\
\hline $\mathrm{C}(21)-\mathrm{Ga}(1)-\mathrm{C}(24)$ & $58.82(18)$ \\
\hline $\mathrm{C}(25)-\mathrm{Ga}(1)-\mathrm{C}(24)$ & $35.43(19)$ \\
\hline $\mathrm{C}(23)-\mathrm{Ga}(1)-\mathrm{C}(22)$ & $35.38(19)$ \\
\hline $\mathrm{C}(21)-\mathrm{Ga}(1)-\mathrm{C}(22)$ & $35.1(2)$ \\
\hline$C(25)-\mathrm{Ga}(1)-\mathrm{C}(22)$ & $58.94(18)$ \\
\hline $\mathrm{C}(24)-\mathrm{Ga}(1)-\mathrm{C}(22)$ & $58.71(17)$ \\
\hline $\mathrm{C}(23)-\mathrm{Ga}(1)-\mathrm{Eu}$ & $150.69(14)$ \\
\hline $\mathrm{C}(21)-\mathrm{Ga}(1)-\mathrm{Eu}$ & $145.95(13)$ \\
\hline $\mathrm{C}(25)-\mathrm{Ga}(1)-\mathrm{Eu}$ & $148.52(13)$ \\
\hline $\mathrm{C}(24)-\mathrm{Ga}(1)-\mathrm{Eu}$ & $151.90(12)$ \\
\hline $\mathrm{C}(22)-\mathrm{Ga}(1)-\mathrm{Eu}$ & $147.10(12)$ \\
\hline$C(32 B)-G a(2)-C(31 B)$ & $36.5(3)$ \\
\hline$C(32 B)-G a(2)-C(33 B)$ & $35.9(3)$ \\
\hline$C(31 B)-G a(2)-C(33 B)$ & $59.5(4)$ \\
\hline$C(32 B)-G a(2)-C(35 A)$ & $58.5(3)$ \\
\hline$C(31 B)-G a(2)-C(35 A)$ & $49.3(3)$ \\
\hline$C(33 B)-G a(2)-C(35 A)$ & $42.8(3)$ \\
\hline$C(32 B)-G a(2)-C(34 A)$ & $46.7(3)$ \\
\hline$C(31 B)-G a(2)-C(34 A)$ & $64.4(3)$ \\
\hline$C(33 B)-G a(2)-C(34 A)$ & $12.2(3)$ \\
\hline$C(35 A)-G a(2)-C(34 A)$ & $35.73(8)$ \\
\hline$C(32 B)-G a(2)-C(35 B)$ & $59.3(4)$ \\
\hline$C(31 B)-G a(2)-C(35 B)$ & $35.4(2)$ \\
\hline$C(33 B)-G a(2)-C(35 B)$ & $58.1(4)$ \\
\hline$C(35 A)-G a(2)-C(35 B)$ & $21.6(3)$ \\
\hline$C(34 A)-G a(2)-C(35 B)$ & $54.5(3)$ \\
\hline $\mathrm{C}(32 \mathrm{~B})-\mathrm{Ga}(2)-\mathrm{C}(31 \mathrm{~A})$ & $46.1(3)$ \\
\hline$C(31 B)-G a(2)-C(31 A)$ & $16.4(3)$ \\
\hline$C(33 B)-G a(2)-C(31 A)$ & $58.0(3)$ \\
\hline$C(35 A)-G a(2)-C(31 A)$ & $35.49(8)$ \\
\hline $\mathrm{C}(34 \mathrm{~A})-\mathrm{Ga}(2)-\mathrm{C}(31 \mathrm{~A})$ & $59.07(12)$ \\
\hline$C(35 B)-G a(2)-C(31 A)$ & $19.0(3)$ \\
\hline$C(32 B)-G a(2)-C(33 A)$ & $17.8(3)$ \\
\hline$C(31 B)-G a(2)-C(33 A)$ & $52.5(3)$ \\
\hline$C(33 B)-G a(2)-C(33 A)$ & $23.3(3)$ \\
\hline$C(35 A)-G a(2)-C(33 A)$ & $59.05(12)$ \\
\hline$C(34 A)-G a(2)-C(33 A)$ & $35.44(7)$ \\
\hline$C(35 B)-G a(2)-C(33 A)$ & $67.1(3)$ \\
\hline$C(31 A)-G a(2)-C(33 A)$ & $58.62(11)$ \\
\hline$C(32 B)-G a(2)-C(32 A)$ & $17.3(3)$ \\
\hline$C(31 B)-G a(2)-C(32 A)$ & $21.4(3)$ \\
\hline$C(33 B)-G a(2)-C(32 A)$ & $49.8(3)$ \\
\hline$C(35 A)-G a(2)-C(32 A)$ & $58.80(11)$ \\
\hline$C(34 A)-G a(2)-C(32 A)$ & $58.76(12)$ \\
\hline$C(35 B)-G a(2)-C(32 A)$ & $52.2(3)$ \\
\hline$C(31 A)-G a(2)-C(32 A)$ & $35.08(7)$ \\
\hline$C(33 A)-G a(2)-C(32 A)$ & $35.05(8)$ \\
\hline$C(32 B)-G a(2)-C(34 B)$ & $58.7(4)$ \\
\hline$C(31 B)-G a(2)-C(34 B)$ & $58.4(4)$ \\
\hline$C(33 B)-G a(2)-C(34 B)$ & $34.6(2)$ \\
\hline$C(35 A)-G a(2)-C(34 B)$ & $12.8(3)$ \\
\hline$C(34 A)-G a(2)-C(34 B)$ & $25.1(3)$ \\
\hline$C(35 B)-G a(2)-C(34 B)$ & $34.4(2)$ \\
\hline$C(31 A)-G a(2)-C(34 B)$ & $46.4(3)$ \\
\hline
\end{tabular}




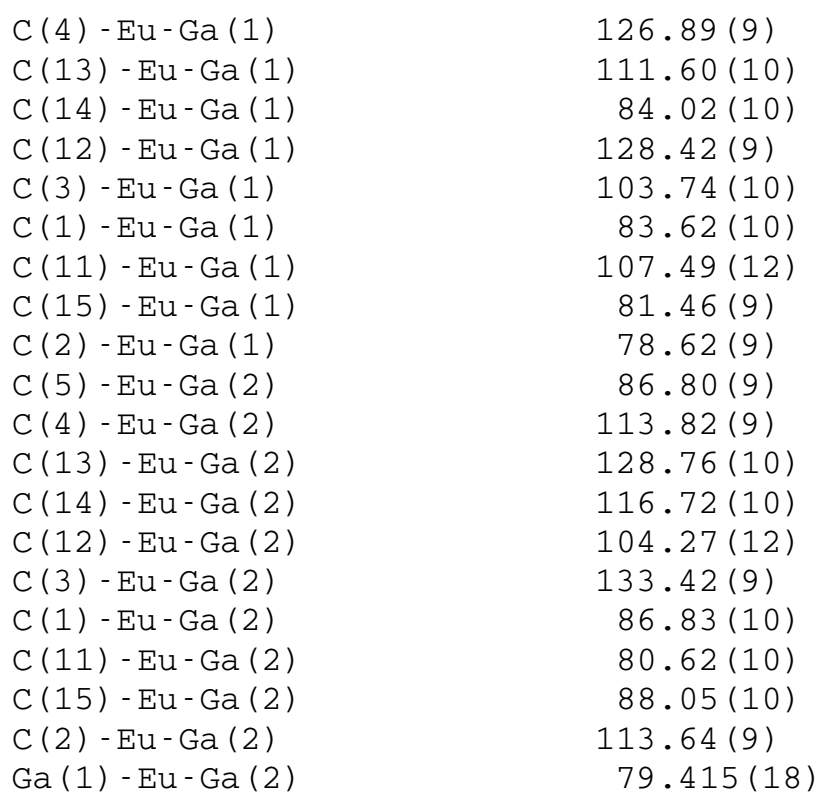

Symmetry transformations used to generate equivalent atoms: 
Table 4. Anisotropic displacement parameters (A^2 x 10^3) for mw33. The anisotropic displacement factor exponent takes the form:

-2 pi^2 [ h^2 a*^2 U11 + .. + $2 h k a * b * U 12]$

\begin{tabular}{|c|c|c|c|c|c|c|}
\hline & U11 & $\mathrm{U} 22$ & U33 & U23 & U13 & $\mathrm{U} 12$ \\
\hline$C(1)$ & $60(3)$ & $54(2)$ & $32(2)$ & $1(2)$ & $4(2)$ & $5(2)$ \\
\hline$C(2)$ & $50(2)$ & $58(3)$ & $36(2)$ & $0(2)$ & $11(2)$ & $-1(2)$ \\
\hline$C(3)$ & $49(2)$ & $58(3)$ & $40(2)$ & $-3(2)$ & $6(2)$ & $7(2)$ \\
\hline$C(4)$ & $51(2)$ & $55(2)$ & $34(2)$ & $-3(2)$ & $4(2)$ & $-3(2)$ \\
\hline$C(5)$ & $43(2)$ & $65(3)$ & $38(2)$ & $-7(2)$ & $4(2)$ & $5(2)$ \\
\hline$C(6)$ & $97(4)$ & $65(3)$ & $51(3)$ & $14(2)$ & $2(3)$ & $12(3)$ \\
\hline$C(7)$ & $70(3)$ & $85(4)$ & $57(3)$ & $-1(3)$ & $24(3)$ & $-12(3)$ \\
\hline$C(8)$ & $65(3)$ & $75(3)$ & $59(3)$ & $-8(3)$ & $8(2)$ & $17(3)$ \\
\hline$C(9)$ & $69(3)$ & $64(3)$ & $54(3)$ & $-11(2)$ & $9(2)$ & $-11(2)$ \\
\hline$C(10)$ & $54(3)$ & $95(4)$ & $69(4)$ & $-15(3)$ & $-2(3)$ & $8(3)$ \\
\hline$C(11)$ & $47(2)$ & $80(3)$ & $38(2)$ & $21(2)$ & $11(2)$ & $11(2)$ \\
\hline$C(12)$ & $63(3)$ & $62(3)$ & $41(2)$ & $12(2)$ & $-4(2)$ & $-15(2)$ \\
\hline C (13) & $67(3)$ & $49(2)$ & $42(2)$ & $4(2)$ & $4(2)$ & $11(2)$ \\
\hline$C(14)$ & $43(2)$ & $64(3)$ & $41(2)$ & $11(2)$ & $3(2)$ & $2(2)$ \\
\hline$C(15)$ & $72(3)$ & $46(2)$ & $32(2)$ & $3(2)$ & $3(2)$ & $3(2)$ \\
\hline$C(16)$ & $79(4)$ & $155(7)$ & $75(4)$ & $52(4)$ & $37(3)$ & $47(4)$ \\
\hline$C(17)$ & $119(6)$ & $107(5)$ & $69(4)$ & $27(4)$ & $-15(4)$ & $-58(5)$ \\
\hline$C(18)$ & $166(7)$ & $65(3)$ & $69(4)$ & $7(3)$ & $18(4)$ & $50(4)$ \\
\hline C (19) & $47(3)$ & $123(5)$ & $82(4)$ & $31(4)$ & $2(3)$ & $-1(3)$ \\
\hline$C(20)$ & $149(6)$ & $57(3)$ & $48(3)$ & $-5(2)$ & $7(3)$ & $-9(3)$ \\
\hline$C(21)$ & $79(3)$ & $58(3)$ & $49(3)$ & $-10(2)$ & $15(2)$ & $-18(3)$ \\
\hline$C(22)$ & $65(3)$ & $63(3)$ & $50(3)$ & $7(2)$ & $-4(2)$ & $-24(2)$ \\
\hline$C(23)$ & $54(3)$ & $61(3)$ & $68(3)$ & $8(3)$ & $8(2)$ & $-10(2)$ \\
\hline$C(24)$ & $70(3)$ & $56(3)$ & $48(3)$ & $5(2)$ & $10(2)$ & $-20(2)$ \\
\hline C (25) & $61(3)$ & $49(2)$ & $64(3)$ & $1(2)$ & $-1(2)$ & $-7(2)$ \\
\hline$C(26)$ & $136(6)$ & $76(4)$ & $73(4)$ & $-24(3)$ & $45(4)$ & $-21(4)$ \\
\hline$C(27)$ & $107(5)$ & $105(5)$ & $77(4)$ & $25(4)$ & $-27(4)$ & $-43(4)$ \\
\hline$C(28)$ & $67(4)$ & $102(5)$ & $127(6)$ & $12(4)$ & $31(4)$ & $-2(4)$ \\
\hline$C(29)$ & $129(6)$ & $82(4)$ & $47(3)$ & $6(3)$ & $10(3)$ & $-35(4)$ \\
\hline$C(30)$ & $94(5)$ & $72(4)$ & $113(6)$ & $-2(4)$ & $-4(4)$ & $22(4)$ \\
\hline $\mathrm{Ga}(1)$ & $61(1)$ & $53(1)$ & $51(1)$ & $0(1)$ & $7(1)$ & $-14(1)$ \\
\hline $\mathrm{Ga}(2)$ & $54(1)$ & $82(1)$ & $49(1)$ & $-4(1)$ & $5(1)$ & $25(1)$ \\
\hline $\mathrm{Eu}$ & $43(1)$ & $42(1)$ & $31(1)$ & $2(1)$ & $4(1)$ & $3(1)$ \\
\hline
\end{tabular}


Table 5. Hydrogen coordinates $(\mathrm{x} 10 \wedge 4)$ and isotropic displacement parameters $(A \wedge 2 \times 10 \wedge 3)$ for 1 .

\begin{tabular}{|c|c|c|c|c|}
\hline & $\mathrm{x}$ & $y$ & $z$ & $\mathrm{U}(\mathrm{eq})$ \\
\hline $\mathrm{H}(6 \mathrm{~A})$ & 2706 & -1381 & -351 & 107 \\
\hline $\mathrm{H}(6 \mathrm{~B})$ & 1931 & -1616 & -295 & 107 \\
\hline $\mathrm{H}(6 \mathrm{C})$ & 2394 & -1162 & 85 & 107 \\
\hline $\mathrm{H}(7 \mathrm{~A})$ & 918 & -841 & -234 & 105 \\
\hline $\mathrm{H}(7 \mathrm{~B})$ & 504 & 81 & -485 & 105 \\
\hline $\mathrm{H}(7 \mathrm{C})$ & 708 & 248 & -5 & 105 \\
\hline $\mathrm{H}(8 \mathrm{~A})$ & 785 & 2405 & -238 & 99 \\
\hline $\mathrm{H}(8 \mathrm{~B})$ & 654 & 2155 & -720 & 99 \\
\hline $\mathrm{H}(8 \mathrm{C})$ & 1157 & 3105 & -572 & 99 \\
\hline $\mathrm{H}(9 \mathrm{~A})$ & 2788 & 3158 & -336 & 93 \\
\hline $\mathrm{H}(9 \mathrm{~B})$ & 2183 & 3434 & -662 & 93 \\
\hline $\mathrm{H}(9 \mathrm{C})$ & 2847 & 2872 & -813 & 93 \\
\hline $\mathrm{H}(10 \mathrm{~A})$ & 3508 & 655 & -686 & 110 \\
\hline $\mathrm{H}(10 \mathrm{~B})$ & 3475 & 71 & -247 & 110 \\
\hline $\mathrm{H}(10 \mathrm{C})$ & 3552 & 1364 & -272 & 110 \\
\hline $\mathrm{H}(16 \mathrm{~A})$ & 2843 & 1031 & -2462 & 153 \\
\hline $\mathrm{H}(16 \mathrm{~B})$ & 2800 & -137 & -2248 & 153 \\
\hline $\mathrm{H}(16 \mathrm{C})$ & 3188 & 837 & -2011 & 153 \\
\hline $\mathrm{H}(17 \mathrm{~A})$ & 2527 & 3349 & -2031 & 149 \\
\hline $\mathrm{H}(17 \mathrm{~B})$ & 3001 & 2622 & -1728 & 149 \\
\hline $\mathrm{H}(17 \mathrm{C})$ & 2439 & 3378 & -1544 & 149 \\
\hline $\mathrm{H}(18 \mathrm{~A})$ & 1325 & 3386 & -1362 & 149 \\
\hline $\mathrm{H}(18 \mathrm{~B})$ & 612 & 2833 & -1465 & 149 \\
\hline $\mathrm{H}(18 \mathrm{C})$ & 953 & 3548 & -1807 & 149 \\
\hline $\mathrm{H}(19 \mathrm{~A})$ & 121 & 859 & -2123 & 126 \\
\hline $\mathrm{H}(19 \mathrm{~B})$ & 130 & 1337 & -1664 & 126 \\
\hline $\mathrm{H}(19 \mathrm{C})$ & 284 & 77 & -1736 & 126 \\
\hline $\mathrm{H}(20 \mathrm{~A})$ & 1001 & -940 & -2196 & 127 \\
\hline $\mathrm{H}(20 \mathrm{~B})$ & 1785 & -1188 & -2142 & 127 \\
\hline $\mathrm{H}(20 \mathrm{C})$ & 1485 & -578 & -2547 & 127 \\
\hline $\mathrm{H}(26 \mathrm{~A})$ & 949 & -4425 & -1998 & 141 \\
\hline $\mathrm{H}(26 \mathrm{~B})$ & 1607 & -3793 & -1829 & 141 \\
\hline $\mathrm{H}(26 \mathrm{C})$ & 1061 & -3185 & -2126 & 141 \\
\hline $\mathrm{H}(27 \mathrm{~A})$ & 67 & -1991 & -2102 & 146 \\
\hline $\mathrm{H}(27 \mathrm{~B})$ & -572 & -1845 & -1833 & 146 \\
\hline $\mathrm{H}(27 \mathrm{C})$ & -440 & -2982 & -2054 & 146 \\
\hline $\mathrm{H}(28 \mathrm{~A})$ & -980 & -2505 & -981 & 146 \\
\hline $\mathrm{H}(28 \mathrm{~B})$ & -711 & -1391 & -1165 & 146 \\
\hline $\mathrm{H}(28 \mathrm{C})$ & -507 & -1725 & -698 & 146 \\
\hline $\mathrm{H}(29 \mathrm{~A})$ & 294 & -2587 & -284 & 129 \\
\hline$H(29 B)$ & 941 & -3349 & -287 & 129 \\
\hline $\mathrm{H}(29 \mathrm{C})$ & 211 & -3874 & -337 & 129 \\
\hline $\mathrm{H}(30 \mathrm{~A})$ & 1880 & -4299 & -1181 & 141 \\
\hline $\mathrm{H}(30 \mathrm{~B})$ & 1416 & -4982 & -890 & 141 \\
\hline $\mathrm{H}(30 \mathrm{C})$ & 1780 & -3902 & -719 & 141 \\
\hline $\mathrm{H}(36 \mathrm{~A})$ & 4291 & -3707 & -670 & 127 \\
\hline $\mathrm{H}(36 \mathrm{~B})$ & 3505 & -3476 & -671 & 127 \\
\hline $\mathrm{H}(36 \mathrm{C})$ & 4024 & -2610 & -472 & 127 \\
\hline $\mathrm{H}(37 \mathrm{~A})$ & 5128 & -857 & -932 & 108 \\
\hline
\end{tabular}




\begin{tabular}{lrrrr}
$H(37 B)$ & 4598 & -1121 & -595 & 108 \\
$H(37 \mathrm{C})$ & 4485 & -99 & -896 & 108 \\
$\mathrm{H}(38 \mathrm{~A})$ & 4430 & 290 & -2087 & 117 \\
$\mathrm{H}(38 \mathrm{~B})$ & 4240 & -239 & -1856 & 117 \\
$\mathrm{H}(38 \mathrm{C})$ & 4950 & -491 & -2387 & 113 \\
$\mathrm{H}(39 \mathrm{~A})$ & 3601 & -1611 & -2311 & 113 \\
$\mathrm{H}(39 \mathrm{~B})$ & 3269 & -2772 & -2388 & 113 \\
$\mathrm{H}(39 \mathrm{C})$ & 4049 & -3975 & -1773 & 123 \\
$\mathrm{H}(40 \mathrm{~A})$ & 3001 & -4353 & -1315 & 123 \\
$\mathrm{H}(40 \mathrm{~B})$ & 3229 & -4554 & -1685 & 123 \\
$\mathrm{H}(40 \mathrm{C})$ & 3710 & -2518 & -477 & 232 \\
$\mathrm{H}(36 \mathrm{D})$ & 4381 & -1587 & -488 & 232 \\
$\mathrm{H}(36 \mathrm{E})$ & 3823 & -1295 & -587 & 232 \\
$\mathrm{H}(36 \mathrm{~F})$ & 4575 & -277 & -1832 & 159 \\
$\mathrm{H}(37 \mathrm{D})$ & 4504 & -179 & -1405 & 159 \\
$\mathrm{H}(37 \mathrm{E})$ & 4941 & 301 & -1444 & 159 \\
$\mathrm{H}(37 \mathrm{~F})$ & 4198 & -2676 & -2341 & 116 \\
$\mathrm{H}(38 \mathrm{D})$ & 4015 & -1439 & -2352 & 116 \\
$\mathrm{H}(38 \mathrm{E})$ & 4261 & -1709 & -2348 & 120 \\
$\mathrm{H}(38 \mathrm{~F})$ & 3480 & -3943 & -2125 & 120 \\
$\mathrm{H}(39 \mathrm{D})$ & 3288 & -4160 & -1693 & 120 \\
$\mathrm{H}(39 \mathrm{E})$ & 2965 & -4591 & -1767 & 218 \\
$\mathrm{H}(39 \mathrm{~F})$ & 3702 & -4688 & -1037 & -747 \\
$\mathrm{H}(40 \mathrm{D})$ & 3517 & -3827 & -683 & \\
$\mathrm{H}(40 \mathrm{E})$ & 3172 & -4068 & & 218 \\
$\mathrm{H}(40 \mathrm{~F})$ & 3956 & & & \\
\hline
\end{tabular}


Table 6. Torsion angles [deg] for 1.

\begin{tabular}{|c|c|}
\hline$C(5)-C(1)-C(2)-C(3)$ & $0.8(5)$ \\
\hline$C(6)-C(1)-C(2)-C(3)$ & $176.5(4)$ \\
\hline$E u-C(1)-C(2)-C(3)$ & $-67.0(3)$ \\
\hline$C(5)-C(1)-C(2)-C(7)$ & $-172.9(5)$ \\
\hline$C(6)-C(1)-C(2)-C(7)$ & $2.7(8)$ \\
\hline$E u-C(1)-C(2)-C(7)$ & $119.2(5)$ \\
\hline$C(5)-C(1)-C(2)-E u$ & $67.9(3)$ \\
\hline$C(6)-C(1)-C(2)-E u$ & $-116.5(5)$ \\
\hline$C(1)-C(2)-C(3)-C(4)$ & $-0.8(5)$ \\
\hline$C(7)-C(2)-C(3)-C(4)$ & $173.2(4)$ \\
\hline$E u-C(2)-C(3)-C(4)$ & $-68.1(3)$ \\
\hline$C(1)-C(2)-C(3)-C(8)$ & $-174.0(4)$ \\
\hline$C(7)-C(2)-C(3)-C(8)$ & $0.0(7)$ \\
\hline$E u-C(2)-C(3)-C(8)$ & $118.7(4)$ \\
\hline$C(1)-C(2)-C(3)-E u$ & $67.3(3)$ \\
\hline$C(7)-C(2)-C(3)-E u$ & $-118.7(4)$ \\
\hline$C(2)-C(3)-C(4)-C(5)$ & $0.4(5)$ \\
\hline$C(8)-C(3)-C(4)-C(5)$ & $173.3(4)$ \\
\hline$E u-C(3)-C(4)-C(5)$ & $-69.4(3)$ \\
\hline$C(2)-C(3)-C(4)-C(9)$ & $-173.8(4)$ \\
\hline$C(8)-C(3)-C(4)-C(9)$ & $-0.9(8)$ \\
\hline$E u-C(3)-C(4)-C(9)$ & $116.5(4)$ \\
\hline$C(2)-C(3)-C(4)-E u$ & $69.8(3)$ \\
\hline$C(8)-C(3)-C(4)-E u$ & $-117.3(5)$ \\
\hline$C(3)-C(4)-C(5)-C(1)$ & $0.1(5)$ \\
\hline$C(9)-C(4)-C(5)-C(1)$ & $174.2(4)$ \\
\hline$E u-C(4)-C(5)-C(1)$ & $-70.1(3)$ \\
\hline$C(3)-C(4)-C(5)-C(10)$ & $-175.9(5)$ \\
\hline$C(9)-C(4)-C(5)-C(10)$ & $-1.8(7)$ \\
\hline$E u-C(4)-C(5)-C(10)$ & $113.9(5)$ \\
\hline$C(3)-C(4)-C(5)-E u$ & $70.2(3)$ \\
\hline$C(9)-C(4)-C(5)-E u$ & $-115.7(4)$ \\
\hline$C(2)-C(1)-C(5)-C(4)$ & $-0.6(5)$ \\
\hline$C(6)-C(1)-C(5)-C(4)$ & $-176.1(4)$ \\
\hline Eu-C (1)-C (5)-C (4) & $69.1(3)$ \\
\hline$C(2)-C(1)-C(5)-C(10)$ & $175.4(4)$ \\
\hline$C(6)-C(1)-C(5)-C(10)$ & $-0.1(7)$ \\
\hline$E u-C(1)-C(5)-C(10)$ & $-114.9(5)$ \\
\hline$C(2)-C(1)-C(5)-E u$ & $-69.6(3)$ \\
\hline$C(6)-C(1)-C(5)-E u$ & $114.8(5)$ \\
\hline$C(15)-C(11)-C(12)-C(13)$ & $-0.1(5)$ \\
\hline$C(16)-C(11)-C(12)-C(13)$ & $172.7(5)$ \\
\hline$E u-C(11)-C(12)-C(13)$ & $-69.1(3)$ \\
\hline$C(15)-C(11)-C(12)-C(17)$ & $-172.2(5)$ \\
\hline$C(16)-C(11)-C(12)-C(17)$ & $0.6(8)$ \\
\hline$E u-C(11)-C(12)-C(17)$ & $118.8(5)$ \\
\hline$C(15)-C(11)-C(12)-E u$ & $69.0(3)$ \\
\hline$C(16)-C(11)-C(12)-E u$ & $-118.2(5)$ \\
\hline$C(11)-C(12)-C(13)-C(14)$ & $0.3(5)$ \\
\hline$C(17)-C(12)-C(13)-C(14)$ & $172.4(5)$ \\
\hline Eu-C (12)-C (13)-C (14) & $-69.4(3)$ \\
\hline$C(11)-C(12)-C(13)-C(18)$ & $-171.7(5)$ \\
\hline$C(17)-C(12)-C(13)-C(18)$ & $0.4(8)$ \\
\hline$E u-C(12)-C(13)-C(18)$ & $118.6(5)$ \\
\hline
\end{tabular}




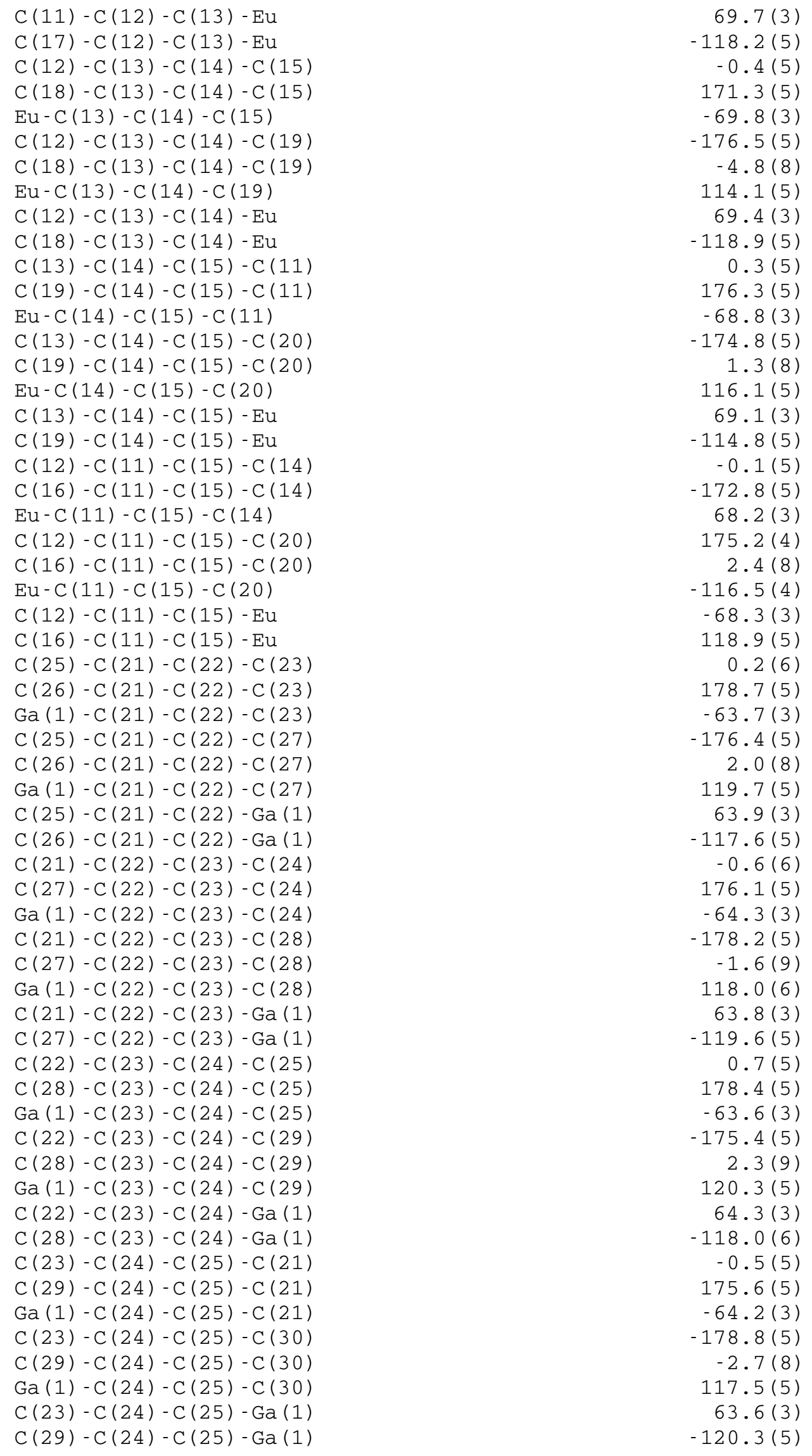




\begin{tabular}{|c|c|}
\hline$C(22)-C(21)-C(25)-C(24)$ & $0.2(5)$ \\
\hline$C(26)-C(21)-C(25)-C(24)$ & $-178.3(5)$ \\
\hline $\mathrm{Ga}(1)-\mathrm{C}(21)-\mathrm{C}(25)-\mathrm{C}(24)$ & $64.3(3)$ \\
\hline$C(22)-C(21)-C(25)-C(30)$ & $178.4(5)$ \\
\hline$C(26)-C(21)-C(25)-C(30)$ & $0.0(9)$ \\
\hline $\mathrm{Ga}(1)-\mathrm{C}(21)-\mathrm{C}(25)-\mathrm{C}(30)$ & $-117.4(6)$ \\
\hline$C(22)-C(21)-C(25)-G a(1)$ & $-64.1(3)$ \\
\hline$C(26)-C(21)-C(25)-G a(1)$ & $117.4(5)$ \\
\hline$C(35 A)-C(31 A)-C(32 A)-C(33 A)$ & 0.0 \\
\hline$C(36 A)-C(31 A)-C(32 A)-C(33 A)$ & 180.0 \\
\hline$G a(2)-C(31 A)-C(32 A)-C(33 A)$ & $-62.99(15)$ \\
\hline$C(35 A)-C(31 A)-C(32 A)-C(37 A)$ & $-175.9(6)$ \\
\hline$C(36 A)-C(31 A)-C(32 A)-C(37 A)$ & $4.1(6)$ \\
\hline $\mathrm{Ga}(2)-\mathrm{C}(31 \mathrm{~A})-\mathrm{C}(32 \mathrm{~A})-\mathrm{C}(37 \mathrm{~A})$ & $121.1(5)$ \\
\hline$C(35 A)-C(31 A)-C(32 A)-G a(2)$ & $62.99(15)$ \\
\hline$C(36 A)-C(31 A)-C(32 A)-G a(2)$ & $-117.01(15)$ \\
\hline$C(31 A)-C(32 A)-C(33 A)-C(34 A)$ & 0.0 \\
\hline$C(37 A)-C(32 A)-C(33 A)-C(34 A)$ & $176.0(6)$ \\
\hline $\mathrm{Ga}(2)-\mathrm{C}(32 \mathrm{~A})-\mathrm{C}(33 \mathrm{~A})-\mathrm{C}(34 \mathrm{~A})$ & $-62.87(17)$ \\
\hline$C(31 A)-C(32 A)-C(33 A)-C(38 A)$ & $-174.5(6)$ \\
\hline$C(37 A)-C(32 A)-C(33 A)-C(38 A)$ & $1.5(7)$ \\
\hline $\mathrm{Ga}(2)-\mathrm{C}(32 \mathrm{~A})-\mathrm{C}(33 \mathrm{~A})-\mathrm{C}(38 \mathrm{~A})$ & $122.6(6)$ \\
\hline$C(31 A)-C(32 A)-C(33 A)-G a(2)$ & $62.87(17)$ \\
\hline$C(37 A)-C(32 A)-C(33 A)-G a(2)$ & $-121.1(5)$ \\
\hline$C(32 A)-C(33 A)-C(34 A)-C(35 A)$ & 0.0 \\
\hline$C(38 A)-C(33 A)-C(34 A)-C(35 A)$ & $174.3(6)$ \\
\hline $\mathrm{Ga}(2)-\mathrm{C}(33 \mathrm{~A})-\mathrm{C}(34 \mathrm{~A})-\mathrm{C}(35 \mathrm{~A})$ & $-64.28(14)$ \\
\hline$C(32 A)-C(33 A)-C(34 A)-C(39 A)$ & $-175.6(6)$ \\
\hline$C(38 A)-C(33 A)-C(34 A)-C(39 A)$ & $-1.3(8)$ \\
\hline $\mathrm{Ga}(2)-\mathrm{C}(33 \mathrm{~A})-\mathrm{C}(34 \mathrm{~A})-\mathrm{C}(39 \mathrm{~A})$ & $120.1(6)$ \\
\hline$C(32 A)-C(33 A)-C(34 A)-G a(2)$ & $64.28(14)$ \\
\hline$C(38 A)-C(33 A)-C(34 A)-G a(2)$ & $-121.4(6)$ \\
\hline$C(32 A)-C(31 A)-C(35 A)-C(34 A)$ & 0.0 \\
\hline$C(36 A)-C(31 A)-C(35 A)-C(34 A)$ & 180.0 \\
\hline $\mathrm{Ga}(2)-\mathrm{C}(31 \mathrm{~A})-\mathrm{C}(35 \mathrm{~A})-\mathrm{C}(34 \mathrm{~A})$ & $64.47(15)$ \\
\hline$C(32 A)-C(31 A)-C(35 A)-C(40 A)$ & $175.4(7)$ \\
\hline$C(36 A)-C(31 A)-C(35 A)-C(40 A)$ & $-4.6(7)$ \\
\hline $\mathrm{Ga}(2)-\mathrm{C}(31 \mathrm{~A})-\mathrm{C}(35 \mathrm{~A})-\mathrm{C}(40 \mathrm{~A})$ & $-120.1(6)$ \\
\hline$C(32 A)-C(31 A)-C(35 A)-G a(2)$ & $-64.46(15)$ \\
\hline$C(36 A)-C(31 A)-C(35 A)-G a(2)$ & $115.53(15)$ \\
\hline$C(33 A)-C(34 A)-C(35 A)-C(31 A)$ & 0.0 \\
\hline$C(39 A)-C(34 A)-C(35 A)-C(31 A)$ & $175.5(6)$ \\
\hline $\mathrm{Ga}(2)-C(34 A)-C(35 A)-C(31 A)$ & $-65.28(17)$ \\
\hline$C(33 A)-C(34 A)-C(35 A)-C(40 A)$ & $-175.6(6)$ \\
\hline$C(39 A)-C(34 A)-C(35 A)-C(40 A)$ & $0.0(8)$ \\
\hline $\mathrm{Ga}(2)-\mathrm{C}(34 \mathrm{~A})-\mathrm{C}(35 \mathrm{~A})-\mathrm{C}(40 \mathrm{~A})$ & $119.2(6)$ \\
\hline$C(33 A)-C(34 A)-C(35 A)-G a(2)$ & $65.28(17)$ \\
\hline$C(39 A)-C(34 A)-C(35 A)-G a(2)$ & $-119.2(6)$ \\
\hline$C(35 B)-C(31 B)-C(32 B)-C(33 B)$ & 0.0 \\
\hline$C(36 B)-C(31 B)-C(32 B)-C(33 B)$ & 180.0 \\
\hline $\mathrm{Ga}(2)-\mathrm{C}(31 \mathrm{~B})-\mathrm{C}(32 \mathrm{~B})-\mathrm{C}(33 \mathrm{~B})$ & $-67.8(5)$ \\
\hline$C(35 B)-C(31 B)-C(32 B)-C(37 B)$ & $175(3)$ \\
\hline$C(36 B)-C(31 B)-C(32 B)-C(37 B)$ & $-5(3)$ \\
\hline $\mathrm{Ga}(2)-\mathrm{C}(31 \mathrm{~B})-\mathrm{C}(32 \mathrm{~B})-\mathrm{C}(37 \mathrm{~B})$ & $107(3)$ \\
\hline$C(35 B)-C(31 B)-C(32 B)-G a(2)$ & $67.8(5)$ \\
\hline$C(36 B)-C(31 B)-C(32 B)-G a(2)$ & $-112.1(5)$ \\
\hline$C(31 B)-C(32 B)-C(33 B)-C(34 B)$ & 0.0 \\
\hline$C(37 B)-C(32 B)-C(33 B)-C(34 B)$ & $-177.0(16)$ \\
\hline
\end{tabular}




$$
\begin{aligned}
& \mathrm{Ga}(2)-\mathrm{C}(32 \mathrm{~B})-\mathrm{C}(33 \mathrm{~B})-\mathrm{C}(34 \mathrm{~B}) \\
& C(31 B)-C(32 B)-C(33 B)-C(38 B) \\
& C(37 B)-C(32 B)-C(33 B)-C(38 B) \\
& \mathrm{Ga}(2)-\mathrm{C}(32 \mathrm{~B})-\mathrm{C}(33 \mathrm{~B})-\mathrm{C}(38 \mathrm{~B}) \\
& C(31 B)-C(32 B)-C(33 B)-G a(2) \\
& C(37 B)-C(32 B)-C(33 B)-G a(2) \\
& C(32 B)-C(33 B)-C(34 B)-C(35 B) \\
& C(38 B)-C(33 B)-C(34 B)-C(35 B) \\
& \mathrm{Ga}(2)-\mathrm{C}(33 \mathrm{~B})-\mathrm{C}(34 \mathrm{~B})-\mathrm{C}(35 \mathrm{~B}) \\
& C(32 B)-C(33 B)-C(34 B)-C(39 B) \\
& C(38 B)-C(33 B)-C(34 B)-C(39 B) \\
& \mathrm{Ga}(2)-\mathrm{C}(33 \mathrm{~B})-\mathrm{C}(34 \mathrm{~B})-\mathrm{C}(39 \mathrm{~B}) \\
& C(32 B)-C(33 B)-C(34 B)-G a(2) \\
& C(38 B)-C(33 B)-C(34 B)-G a(2) \\
& C(33 B)-C(34 B)-C(35 B)-C(31 B) \\
& C(39 B)-C(34 B)-C(35 B)-C(31 B) \\
& \mathrm{Ga}(2)-\mathrm{C}(34 \mathrm{~B})-\mathrm{C}(35 \mathrm{~B})-\mathrm{C}(31 \mathrm{~B}) \\
& C(33 B)-C(34 B)-C(35 B)-C(40 B) \\
& C(39 B)-C(34 B)-C(35 B)-C(40 B) \\
& \mathrm{Ga}(2)-\mathrm{C}(34 \mathrm{~B})-\mathrm{C}(35 \mathrm{~B})-\mathrm{C}(40 \mathrm{~B}) \\
& C(33 B)-C(34 B)-C(35 B)-G a(2) \\
& C(39 B)-C(34 B)-C(35 B)-G a(2) \\
& C(32 B)-C(31 B)-C(35 B)-C(34 B) \\
& C(36 B)-C(31 B)-C(35 B)-C(34 B) \\
& \mathrm{Ga}(2)-\mathrm{C}(31 \mathrm{~B})-\mathrm{C}(35 \mathrm{~B})-\mathrm{C}(34 \mathrm{~B}) \\
& C(32 B)-C(31 B)-C(35 B)-C(40 B) \\
& C(36 B)-C(31 B)-C(35 B)-C(40 B) \\
& \mathrm{Ga}(2)-\mathrm{C}(31 \mathrm{~B})-\mathrm{C}(35 \mathrm{~B})-\mathrm{C}(40 \mathrm{~B}) \\
& C(32 B)-C(31 B)-C(35 B)-G a(2) \\
& C(36 B)-C(31 B)-C(35 B)-G a(2) \\
& \mathrm{C}(24)-\mathrm{C}(23)-\mathrm{Ga}(1)-\mathrm{C}(21) \\
& \mathrm{C}(22)-\mathrm{C}(23)-\mathrm{Ga}(1)-\mathrm{C}(21) \\
& C(28)-C(23)-G a(1)-C(21) \\
& \mathrm{C}(24)-\mathrm{C}(23)-\mathrm{Ga}(1)-\mathrm{C}(25) \\
& C(22)-C(23)-G a(1)-C(25) \\
& \mathrm{C}(28)-\mathrm{C}(23)-\mathrm{Ga}(1)-\mathrm{C}(25) \\
& \mathrm{C}(22)-\mathrm{C}(23)-\mathrm{Ga}(1)-\mathrm{C}(24) \\
& \mathrm{C}(28)-\mathrm{C}(23)-\mathrm{Ga}(1)-\mathrm{C}(24) \\
& C(24)-C(23)-G a(1)-C(22) \\
& \mathrm{C}(28)-\mathrm{C}(23)-\mathrm{Ga}(1)-\mathrm{C}(22) \\
& \mathrm{C}(24)-\mathrm{C}(23)-\mathrm{Ga}(1)-\mathrm{Eu} \\
& \mathrm{C}(22)-\mathrm{C}(23)-\mathrm{Ga}(1)-\mathrm{Eu} \\
& C(28)-C(23)-G a(1)-E u \\
& \mathrm{C}(22)-\mathrm{C}(21)-\mathrm{Ga}(1)-\mathrm{C}(23) \\
& \mathrm{C}(25)-\mathrm{C}(21)-\mathrm{Ga}(1)-\mathrm{C}(23) \\
& C(26)-C(21)-G a(1)-C(23) \\
& \mathrm{C}(22)-\mathrm{C}(21)-\mathrm{Ga}(1)-\mathrm{C}(25) \\
& \mathrm{C}(26)-\mathrm{C}(21)-\mathrm{Ga}(1)-\mathrm{C}(25) \\
& C(22)-C(21)-G a(1)-C(24) \\
& \mathrm{C}(25)-\mathrm{C}(21)-\mathrm{Ga}(1)-\mathrm{C}(24) \\
& \mathrm{C}(26)-\mathrm{C}(21)-\mathrm{Ga}(1)-\mathrm{C}(24) \\
& \mathrm{C}(25)-\mathrm{C}(21)-\mathrm{Ga}(1)-\mathrm{C}(22) \\
& \mathrm{C}(26)-\mathrm{C}(21)-\mathrm{Ga}(1)-\mathrm{C}(22) \\
& \mathrm{C}(22)-\mathrm{C}(21)-\mathrm{Ga}(1)-\mathrm{Eu} \\
& C(25)-C(21)-G a(1)-E u \\
& C(26)-C(21)-\mathrm{Ga}(1)-\mathrm{Eu} \\
& \mathrm{C}(24)-\mathrm{C}(25)-\mathrm{Ga}(1)-\mathrm{C}(23) \\
& \mathrm{C}(21)-\mathrm{C}(25)-\mathrm{Ga}(1)-\mathrm{C}(23)
\end{aligned}
$$

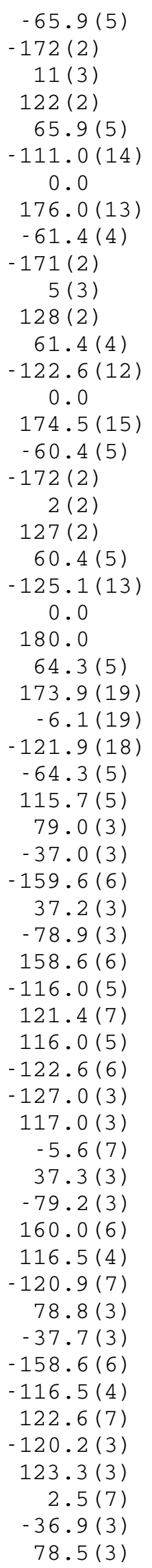




\begin{tabular}{|c|c|}
\hline $\mathrm{C}(30)-\mathrm{C}(25)-\mathrm{Ga}(1)-\mathrm{C}(23)$ & $-158.7(6)$ \\
\hline $\mathrm{C}(24)-\mathrm{C}(25)-\mathrm{Ga}(1)-\mathrm{C}(21)$ & $-115.4(4)$ \\
\hline $\mathrm{C}(30)-\mathrm{C}(25)-\mathrm{Ga}(1)-\mathrm{C}(21)$ & $122.8(7)$ \\
\hline $\mathrm{C}(21)-\mathrm{C}(25)-\mathrm{Ga}(1)-\mathrm{C}(24)$ & $115.4(4)$ \\
\hline $\mathrm{C}(30)-\mathrm{C}(25)-\mathrm{Ga}(1)-\mathrm{C}(24)$ & $-121.7(6)$ \\
\hline $\mathrm{C}(24)-\mathrm{C}(25)-\mathrm{Ga}(1)-\mathrm{C}(22)$ & $-78.5(3)$ \\
\hline $\mathrm{C}(21)-\mathrm{C}(25)-\mathrm{Ga}(1)-\mathrm{C}(22)$ & $36.9(3)$ \\
\hline $\mathrm{C}(30)-\mathrm{C}(25)-\mathrm{Ga}(1)-\mathrm{C}(22)$ & $159.8(6)$ \\
\hline $\mathrm{C}(24)-\mathrm{C}(25)-\mathrm{Ga}(1)-\mathrm{Eu}$ & $128.2(3)$ \\
\hline $\mathrm{C}(21)-\mathrm{C}(25)-\mathrm{Ga}(1)-\mathrm{Eu}$ & $-116.4(3)$ \\
\hline $\mathrm{C}(30)-\mathrm{C}(25)-\mathrm{Ga}(1)-\mathrm{Eu}$ & $6.5(7)$ \\
\hline $\mathrm{C}(25)-\mathrm{C}(24)-\mathrm{Ga}(1)-\mathrm{C}(23)$ & $116.7(4)$ \\
\hline $\mathrm{C}(29)-\mathrm{C}(24)-\mathrm{Ga}(1)-\mathrm{C}(23)$ & $-122.5(7)$ \\
\hline $\mathrm{C}(23)-\mathrm{C}(24)-\mathrm{Ga}(1)-\mathrm{C}(21)$ & $-78.8(3)$ \\
\hline $\mathrm{C}(25)-\mathrm{C}(24)-\mathrm{Ga}(1)-\mathrm{C}(21)$ & $37.9(3)$ \\
\hline $\mathrm{C}(29)-\mathrm{C}(24)-\mathrm{Ga}(1)-\mathrm{C}(21)$ & $158.7(6)$ \\
\hline $\mathrm{C}(23)-\mathrm{C}(24)-\mathrm{Ga}(1)-\mathrm{C}(25)$ & $-116.7(4)$ \\
\hline $\mathrm{C}(29)-\mathrm{C}(24)-\mathrm{Ga}(1)-\mathrm{C}(25)$ & $120.8(7)$ \\
\hline $\mathrm{C}(23)-\mathrm{C}(24)-\mathrm{Ga}(1)-\mathrm{C}(22)$ & $-37.5(3)$ \\
\hline $\mathrm{C}(25)-\mathrm{C}(24)-\mathrm{Ga}(1)-\mathrm{C}(22)$ & $79.2(3)$ \\
\hline $\mathrm{C}(29)-\mathrm{C}(24)-\mathrm{Ga}(1)-\mathrm{C}(22)$ & $-160.0(6)$ \\
\hline $\mathrm{C}(23)-\mathrm{C}(24)-\mathrm{Ga}(1)-\mathrm{Eu}$ & $123.9(3)$ \\
\hline $\mathrm{C}(25)-\mathrm{C}(24)-\mathrm{Ga}(1)-\mathrm{Eu}$ & $-119.4(3)$ \\
\hline $\mathrm{C}(29)-\mathrm{C}(24)-\mathrm{Ga}(1)-\mathrm{Eu}$ & $1.4(7)$ \\
\hline $\mathrm{C}(21)-\mathrm{C}(22)-\mathrm{Ga}(1)-\mathrm{C}(23)$ & $-116.4(4)$ \\
\hline $\mathrm{C}(27)-\mathrm{C}(22)-\mathrm{Ga}(1)-\mathrm{C}(23)$ & $121.7(7)$ \\
\hline $\mathrm{C}(23)-\mathrm{C}(22)-\mathrm{Ga}(1)-\mathrm{C}(21)$ & $116.4(4)$ \\
\hline $\mathrm{C}(27)-\mathrm{C}(22)-\mathrm{Ga}(1)-\mathrm{C}(21)$ & $-121.9(7)$ \\
\hline$C(21)-C(22)-\mathrm{Ga}(1)-\mathrm{C}(25)$ & $-37.4(3)$ \\
\hline $\mathrm{C}(23)-\mathrm{C}(22)-\mathrm{Ga}(1)-\mathrm{C}(25)$ & $79.0(3)$ \\
\hline$C(27)-C(22)-\mathrm{Ga}(1)-\mathrm{C}(25)$ & $-159.3(6)$ \\
\hline $\mathrm{C}(21)-\mathrm{C}(22)-\mathrm{Ga}(1)-\mathrm{C}(24)$ & $-79.1(3)$ \\
\hline $\mathrm{C}(23)-\mathrm{C}(22)-\mathrm{Ga}(1)-\mathrm{C}(24)$ & $37.3(3)$ \\
\hline $\mathrm{C}(27)-\mathrm{C}(22)-\mathrm{Ga}(1)-\mathrm{C}(24)$ & $159.0(7)$ \\
\hline $\mathrm{C}(21)-\mathrm{C}(22)-\mathrm{Ga}(1)-\mathrm{Eu}$ & $117.0(3)$ \\
\hline $\mathrm{C}(23)-\mathrm{C}(22)-\mathrm{Ga}(1)-\mathrm{Eu}$ & $-126.6(3)$ \\
\hline$C(27)-C(22)-\mathrm{Ga}(1)-\mathrm{Eu}$ & $-4.9(7)$ \\
\hline$C(33 B)-C(32 B)-G a(2)-C(31 B)$ & $114.5(2)$ \\
\hline$C(37 B)-C(32 B)-G a(2)-C(31 B)$ & $-145.6(17)$ \\
\hline$C(31 B)-C(32 B)-G a(2)-C(33 B)$ & $-114.5(2)$ \\
\hline$C(37 B)-C(32 B)-G a(2)-C(33 B)$ & $99.8(17)$ \\
\hline$C(33 B)-C(32 B)-G a(2)-C(35 A)$ & $51.6(4)$ \\
\hline$C(31 B)-C(32 B)-G a(2)-C(35 A)$ & $-62.9(4)$ \\
\hline$C(37 B)-C(32 B)-G a(2)-C(35 A)$ & $151.5(16)$ \\
\hline$C(33 B)-C(32 B)-G a(2)-C(34 A)$ & $8.5(5)$ \\
\hline$C(31 B)-C(32 B)-G a(2)-C(34 A)$ & $-106.0(4)$ \\
\hline$C(37 B)-C(32 B)-G a(2)-C(34 A)$ & $108.4(16)$ \\
\hline$C(33 B)-C(32 B)-G a(2)-C(35 B)$ & $76.9(3)$ \\
\hline$C(31 B)-C(32 B)-G a(2)-C(35 B)$ & $-37.64(16)$ \\
\hline$C(37 B)-C(32 B)-G a(2)-C(35 B)$ & $176.7(17)$ \\
\hline$C(33 B)-C(32 B)-G a(2)-C(31 A)$ & $94.3(4)$ \\
\hline$C(31 B)-C(32 B)-G a(2)-C(31 A)$ & $-20.2(4)$ \\
\hline$C(37 B)-C(32 B)-G a(2)-C(31 A)$ & $-165.9(17)$ \\
\hline$C(33 B)-C(32 B)-G a(2)-C(33 A)$ & $-34.8(11)$ \\
\hline$C(31 B)-C(32 B)-G a(2)-C(33 A)$ & $-149.4(11)$ \\
\hline$C(37 B)-C(32 B)-G a(2)-C(33 A)$ & $65.0(17)$ \\
\hline$C(33 B)-C(32 B)-G a(2)-C(32 A)$ & $137.4(10)$ \\
\hline$C(31 B)-C(32 B)-G a(2)-C(32 A)$ & $22.9(10)$ \\
\hline
\end{tabular}




\begin{tabular}{|c|c|}
\hline$C(37 B)-C(32 B)-G a(2)-C(32 A)$ & $-123(2)$ \\
\hline$C(33 B)-C(32 B)-G a(2)-C(34 B)$ & $36.57(15)$ \\
\hline$C(31 B)-C(32 B)-G a(2)-C(34 B)$ & $-78.0(3)$ \\
\hline$C(37 B)-C(32 B)-G a(2)-C(34 B)$ & $136.4(17)$ \\
\hline$C(33 B)-C(32 B)-G a(2)-E u$ & $-127.5(4)$ \\
\hline$C(31 B)-C(32 B)-G a(2)-E u$ & $118.0(4)$ \\
\hline$C(37 B)-C(32 B)-G a(2)-E u$ & $-27.6(17)$ \\
\hline$C(35 B)-C(31 B)-G a(2)-C(32 B)$ & $-114.9(2)$ \\
\hline$C(36 B)-C(31 B)-G a(2)-C(32 B)$ & $121.3(2)$ \\
\hline$C(35 B)-C(31 B)-G a(2)-C(33 B)$ & $-76.7(3)$ \\
\hline$C(32 B)-C(31 B)-G a(2)-C(33 B)$ & $38.20(18)$ \\
\hline$C(36 B)-C(31 B)-G a(2)-C(33 B)$ & $159.50(9)$ \\
\hline$C(35 B)-C(31 B)-G a(2)-C(35 A)$ & $-24.8(4)$ \\
\hline$C(32 B)-C(31 B)-G a(2)-C(35 A)$ & $90.1(4)$ \\
\hline$C(36 B)-C(31 B)-G a(2)-C(35 A)$ & $-148.6(4)$ \\
\hline$C(35 B)-C(31 B)-G a(2)-C(34 A)$ & $-64.0(4)$ \\
\hline$C(32 B)-C(31 B)-G a(2)-C(34 A)$ & $50.9(4)$ \\
\hline$C(36 B)-C(31 B)-G a(2)-C(34 A)$ & $172.2(4)$ \\
\hline$C(32 B)-C(31 B)-G a(2)-C(35 B)$ & $114.9(2)$ \\
\hline$C(36 B)-C(31 B)-G a(2)-C(35 B)$ & $-123.8(2)$ \\
\hline$C(35 B)-C(31 B)-G a(2)-C(31 A)$ & $3.0(10)$ \\
\hline$C(32 B)-C(31 B)-G a(2)-C(31 A)$ & $117.9(10)$ \\
\hline$C(36 B)-C(31 B)-G a(2)-C(31 A)$ & $-120.8(10)$ \\
\hline$C(35 B)-C(31 B)-G a(2)-C(33 A)$ & $-103.6(4)$ \\
\hline$C(32 B)-C(31 B)-G a(2)-C(33 A)$ & $11.3(4)$ \\
\hline$C(36 B)-C(31 B)-G a(2)-C(33 A)$ & $132.6(4)$ \\
\hline$C(35 B)-C(31 B)-G a(2)-C(32 A)$ & $-133.4(8)$ \\
\hline$C(32 B)-C(31 B)-G a(2)-C(32 A)$ & $-18.5(9)$ \\
\hline$C(36 B)-C(31 B)-G a(2)-C(32 A)$ & $102.8(8)$ \\
\hline$C(35 B)-C(31 B)-G a(2)-C(34 B)$ & $-36.14(18)$ \\
\hline$C(32 B)-C(31 B)-G a(2)-C(34 B)$ & $78.8(3)$ \\
\hline$C(36 B)-C(31 B)-G a(2)-C(34 B)$ & $-159.91(9)$ \\
\hline$C(35 B)-C(31 B)-G a(2)-E u$ & $141.6(4)$ \\
\hline$C(32 B)-C(31 B)-G a(2)-E u$ & $-103.5(4)$ \\
\hline$C(36 B)-C(31 B)-G a(2)-E u$ & $17.8(4)$ \\
\hline$C(34 B)-C(33 B)-G a(2)-C(32 B)$ & $116.3(3)$ \\
\hline$C(38 B)-C(33 B)-G a(2)-C(32 B)$ & $-148.1(16)$ \\
\hline$C(34 B)-C(33 B)-G a(2)-C(31 B)$ & $77.4(3)$ \\
\hline$C(32 B)-C(33 B)-G a(2)-C(31 B)$ & $-38.86(19)$ \\
\hline$C(38 B)-C(33 B)-G a(2)-C(31 B)$ & $173.0(16)$ \\
\hline$C(34 B)-C(33 B)-G a(2)-C(35 A)$ & $15.9(5)$ \\
\hline$C(32 B)-C(33 B)-G a(2)-C(35 A)$ & $-100.4(4)$ \\
\hline$C(38 B)-C(33 B)-G a(2)-C(35 A)$ & $111.5(16)$ \\
\hline$C(34 B)-C(33 B)-G a(2)-C(34 A)$ & $-33.0(16)$ \\
\hline$C(32 B)-C(33 B)-G a(2)-C(34 A)$ & $-149.3(16)$ \\
\hline$C(38 B)-C(33 B)-G a(2)-C(34 A)$ & $62.6(19)$ \\
\hline$C(34 B)-C(33 B)-G a(2)-C(35 B)$ & $35.87(19)$ \\
\hline$C(32 B)-C(33 B)-G a(2)-C(35 B)$ & $-80.4(3)$ \\
\hline$C(38 B)-C(33 B)-G a(2)-C(35 B)$ & $131.4(16)$ \\
\hline$C(34 B)-C(33 B)-G a(2)-C(31 A)$ & $58.3(4)$ \\
\hline$C(32 B)-C(33 B)-G a(2)-C(31 A)$ & $-58.0(3)$ \\
\hline$C(38 B)-C(33 B)-G a(2)-C(31 A)$ & $153.9(16)$ \\
\hline$C(34 B)-C(33 B)-G a(2)-C(33 A)$ & $142.5(9)$ \\
\hline$C(32 B)-C(33 B)-G a(2)-C(33 A)$ & $26.2(9)$ \\
\hline$C(38 B)-C(33 B)-G a(2)-C(33 A)$ & $-121.9(18)$ \\
\hline$C(34 B)-C(33 B)-G a(2)-C(32 A)$ & $101.0(4)$ \\
\hline$C(32 B)-C(33 B)-G a(2)-C(32 A)$ & $-15.3(4)$ \\
\hline$C(38 B)-C(33 B)-G a(2)-C(32 A)$ & $-163.4(16)$ \\
\hline
\end{tabular}




\begin{tabular}{|c|c|}
\hline$C(32 B)-C(33 B)-G a(2)-C(34 B)$ & $-116.3(3)$ \\
\hline$C(38 B)-C(33 B)-G a(2)-C(34 B)$ & $95.6(17)$ \\
\hline$C(34 B)-C(33 B)-G a(2)-E u$ & $-147.4(5)$ \\
\hline$C(32 B)-C(33 B)-G a(2)-E u$ & $96.3(4)$ \\
\hline$C(38 B)-C(33 B)-G a(2)-E u$ & $-51.8(17)$ \\
\hline$C(31 A)-C(35 A)-G a(2)-C(32 B)$ & $57.3(4)$ \\
\hline$C(34 A)-C(35 A)-G a(2)-C(32 B)$ & $-58.3(4)$ \\
\hline$C(40 A)-C(35 A)-G a(2)-C(32 B)$ & $-178.7(7)$ \\
\hline$C(31 A)-C(35 A)-G a(2)-C(31 B)$ & $13.1(4)$ \\
\hline$C(34 A)-C(35 A)-G a(2)-C(31 B)$ & $-102.6(4)$ \\
\hline$C(40 A)-C(35 A)-G a(2)-C(31 B)$ & $137.1(8)$ \\
\hline$C(31 A)-C(35 A)-G a(2)-C(33 B)$ & $99.9(5)$ \\
\hline$C(34 A)-C(35 A)-G a(2)-C(33 B)$ & $-15.8(5)$ \\
\hline$C(40 A)-C(35 A)-G a(2)-C(33 B)$ & $-136.1(8)$ \\
\hline$C(31 A)-C(35 A)-G a(2)-C(34 A)$ & $115.69(8)$ \\
\hline$C(40 A)-C(35 A)-G a(2)-C(34 A)$ & $-120.3(6)$ \\
\hline$C(31 A)-C(35 A)-G a(2)-C(35 B)$ & $-28.2(9)$ \\
\hline$C(34 A)-C(35 A)-G a(2)-C(35 B)$ & $-143.9(9)$ \\
\hline$C(40 A)-C(35 A)-G a(2)-C(35 B)$ & $95.8(11)$ \\
\hline$C(34 A)-C(35 A)-G a(2)-C(31 A)$ & $-115.69(8)$ \\
\hline$C(40 A)-C(35 A)-G a(2)-C(31 A)$ & $124.0(6)$ \\
\hline$C(31 A)-C(35 A)-G a(2)-C(33 A)$ & $78.18(9)$ \\
\hline$C(34 A)-C(35 A)-G a(2)-C(33 A)$ & -37.5 \\
\hline$C(40 A)-C(35 A)-G a(2)-C(33 A)$ & $-157.8(6)$ \\
\hline$C(31 A)-C(35 A)-G a(2)-C(32 A)$ & $37.01(5)$ \\
\hline$C(34 A)-C(35 A)-G a(2)-C(32 A)$ & $-78.68(8)$ \\
\hline$C(40 A)-C(35 A)-G a(2)-C(32 A)$ & $161.0(6)$ \\
\hline$C(31 A)-C(35 A)-G a(2)-C(34 B)$ & $144.4(16)$ \\
\hline$C(34 A)-C(35 A)-G a(2)-C(34 B)$ & $28.7(16)$ \\
\hline$C(40 A)-C(35 A)-G a(2)-C(34 B)$ & $-91.6(17)$ \\
\hline$C(31 A)-C(35 A)-G a(2)-E u$ & $-125.4(4)$ \\
\hline$C(34 A)-C(35 A)-G a(2)-E u$ & $118.9(4)$ \\
\hline$C(40 A)-C(35 A)-G a(2)-E u$ & $-1.4(8)$ \\
\hline$C(35 A)-C(34 A)-G a(2)-C(32 B)$ & $94.5(4)$ \\
\hline$C(33 A)-C(34 A)-G a(2)-C(32 B)$ & $-21.2(4)$ \\
\hline$C(39 A)-C(34 A)-G a(2)-C(32 B)$ & $-143.2(8)$ \\
\hline$C(35 A)-C(34 A)-G a(2)-C(31 B)$ & $55.2(3)$ \\
\hline$C(33 A)-C(34 A)-G a(2)-C(31 B)$ & $-60.5(3)$ \\
\hline$C(39 A)-C(34 A)-G a(2)-C(31 B)$ & $177.5(7)$ \\
\hline$C(35 A)-C(34 A)-G a(2)-C(33 B)$ & $118.8(16)$ \\
\hline$C(33 A)-C(34 A)-G a(2)-C(33 B)$ & $3.1(16)$ \\
\hline$C(39 A)-C(34 A)-G a(2)-C(33 B)$ & $-118.9(17)$ \\
\hline$C(33 A)-C(34 A)-G a(2)-C(35 A)$ & $-115.75(7)$ \\
\hline$C(39 A)-C(34 A)-G a(2)-C(35 A)$ & $122.3(6)$ \\
\hline$C(35 A)-C(34 A)-G a(2)-C(35 B)$ & $15.4(4)$ \\
\hline$C(33 A)-C(34 A)-G a(2)-C(35 B)$ & $-100.3(4)$ \\
\hline$C(39 A)-C(34 A)-G a(2)-C(35 B)$ & $137.8(7)$ \\
\hline$C(35 A)-C(34 A)-G a(2)-C(31 A)$ & $37.58(5)$ \\
\hline$C(33 A)-C(34 A)-G a(2)-C(31 A)$ & $-78.16(9)$ \\
\hline$C(39 A)-C(34 A)-G a(2)-C(31 A)$ & $159.9(6)$ \\
\hline$C(35 A)-C(34 A)-G a(2)-C(33 A)$ & $115.75(7)$ \\
\hline$C(39 A)-C(34 A)-G a(2)-C(33 A)$ & $-121.9(6)$ \\
\hline$C(35 A)-C(34 A)-G a(2)-C(32 A)$ & $78.79(8)$ \\
\hline$C(33 A)-C(34 A)-G a(2)-C(32 A)$ & $-36.96(6)$ \\
\hline$C(39 A)-C(34 A)-G a(2)-C(32 A)$ & $-158.9(6)$ \\
\hline$C(35 A)-C(34 A)-G a(2)-C(34 B)$ & $-14.5(8)$ \\
\hline$C(33 A)-C(34 A)-G a(2)-C(34 B)$ & $-130.3(8)$ \\
\hline$C(39 A)-C(34 A)-G a(2)-C(34 B)$ & $107.8(10)$ \\
\hline
\end{tabular}




$$
\begin{aligned}
& C(35 A)-C(34 A)-G a(2)-E u \\
& C(33 A)-C(34 A)-G a(2)-E u \\
& C(39 A)-C(34 A)-G a(2)-E u \\
& C(34 B)-C(35 B)-G a(2)-C(32 B) \\
& C(31 B)-C(35 B)-G a(2)-C(32 B) \\
& C(40 B)-C(35 B)-G a(2)-C(32 B) \\
& C(34 B)-C(35 B)-G a(2)-C(31 B) \\
& C(40 B)-C(35 B)-G a(2)-C(31 B) \\
& C(34 B)-C(35 B)-G a(2)-C(33 B) \\
& C(31 B)-C(35 B)-G a(2)-C(33 B) \\
& C(40 B)-C(35 B)-G a(2)-C(33 B) \\
& C(34 B)-C(35 B)-G a(2)-C(35 A) \\
& C(31 B)-C(35 B)-G a(2)-C(35 A) \\
& C(40 B)-C(35 B)-G a(2)-C(35 A) \\
& C(34 B)-C(35 B)-G a(2)-C(34 A) \\
& C(31 B)-C(35 B)-G a(2)-C(34 A) \\
& C(40 B)-C(35 B)-G a(2)-C(34 A) \\
& C(34 B)-C(35 B)-G a(2)-C(31 A) \\
& C(31 B)-C(35 B)-G a(2)-C(31 A) \\
& C(40 B)-C(35 B)-G a(2)-C(31 A) \\
& C(34 B)-C(35 B)-G a(2)-C(33 A) \\
& C(31 B)-C(35 B)-G a(2)-C(33 A) \\
& C(40 B)-C(35 B)-G a(2)-C(33 A) \\
& C(34 B)-C(35 B)-G a(2)-C(32 A) \\
& C(31 B)-C(35 B)-G a(2)-C(32 A) \\
& C(40 B)-C(35 B)-G a(2)-C(32 A) \\
& C(31 B)-C(35 B)-G a(2)-C(34 B) \\
& C(40 B)-C(35 B)-G a(2)-C(34 B) \\
& C(34 B)-C(35 B)-G a(2)-E u \\
& C(31 B)-C(35 B)-G a(2)-E u \\
& C(40 B)-C(35 B)-G a(2)-E u \\
& C(35 A)-C(31 A)-G a(2)-C(32 B) \\
& C(32 A)-C(31 A)-G a(2)-C(32 B) \\
& C(36 A)-C(31 A)-G a(2)-C(32 B) \\
& C(35 A)-C(31 A)-G a(2)-C(31 B) \\
& C(32 A)-C(31 A)-G a(2)-C(31 B) \\
& C(36 A)-C(31 A)-G a(2)-C(31 B) \\
& C(35 A)-C(31 A)-G a(2)-C(33 B) \\
& C(32 A)-C(31 A)-G a(2)-C(33 B) \\
& C(36 A)-C(31 A)-G a(2)-C(33 B) \\
& C(32 A)-C(31 A)-G a(2)-C(35 A) \\
& C(36 A)-C(31 A)-G a(2)-C(35 A) \\
& C(35 A)-C(31 A)-G a(2)-C(34 A) \\
& C(32 A)-C(31 A)-G a(2)-C(34 A) \\
& C(36 A)-C(31 A)-G a(2)-C(34 A) \\
& C(35 A)-C(31 A)-G a(2)-C(35 B) \\
& C(32 A)-C(31 A)-G a(2)-C(35 B) \\
& C(36 A)-C(31 A)-G a(2)-C(35 B) \\
& C(35 A)-C(31 A)-G a(2)-C(33 A) \\
& C(32 A)-C(31 A)-G a(2)-C(33 A) \\
& C(36 A)-C(31 A)-G a(2)-C(33 A) \\
& C(35 A)-C(31 A)-G a(2)-C(32 A) \\
& C(36 A)-C(31 A)-G a(2)-C(32 A) \\
& C(35 A)-C(31 A)-G a(2)-C(34 B) \\
& C(32 A)-C(31 A)-G a(2)-C(34 B) \\
& C(36 A)-C(31 A)-G a(2)-C(34 B) \\
& C(35 A)-C(31 A)-G a(2)-E u \\
& C(32 A)-C(31 A)-G a(2)-E u
\end{aligned}
$$

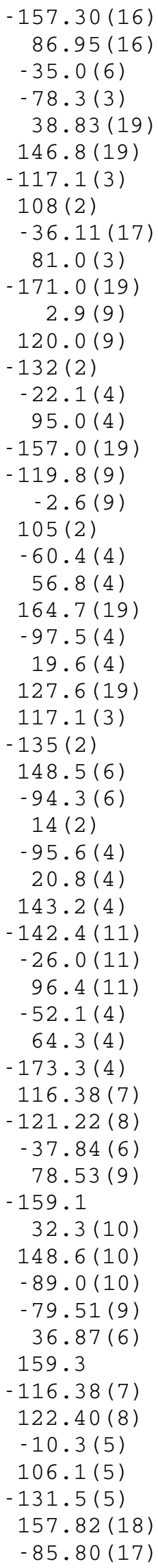




$$
\begin{aligned}
& C(36 A)-C(31 A)-G a(2)-E u \\
& C(32 A)-C(33 A)-G a(2)-C(32 B) \\
& C(34 A)-C(33 A)-G a(2)-C(32 B) \\
& C(38 A)-C(33 A)-G a(2)-C(32 B) \\
& C(32 A)-C(33 A)-G a(2)-C(31 B) \\
& C(34 A)-C(33 A)-G a(2)-C(31 B) \\
& C(38 A)-C(33 A)-G a(2)-C(31 B) \\
& C(32 A)-C(33 A)-G a(2)-C(33 B) \\
& C(34 A)-C(33 A)-G a(2)-C(33 B) \\
& C(38 A)-C(33 A)-G a(2)-C(33 B) \\
& C(32 A)-C(33 A)-G a(2)-C(35 A) \\
& C(34 A)-C(33 A)-G a(2)-C(35 A) \\
& C(38 A)-C(33 A)-G a(2)-C(35 A) \\
& C(32 A)-C(33 A)-G a(2)-C(34 A) \\
& C(38 A)-C(33 A)-G a(2)-C(34 A) \\
& C(32 A)-C(33 A)-G a(2)-C(35 B) \\
& C(34 A)-C(33 A)-G a(2)-C(35 B) \\
& C(38 A)-C(33 A)-G a(2)-C(35 B) \\
& C(32 A)-C(33 A)-G a(2)-C(31 A) \\
& C(34 A)-C(33 A)-G a(2)-C(31 A) \\
& C(38 A)-C(33 A)-G a(2)-C(31 A) \\
& C(34 A)-C(33 A)-G a(2)-C(32 A) \\
& C(38 A)-C(33 A)-G a(2)-C(32 A) \\
& C(32 A)-C(33 A)-G a(2)-C(34 B) \\
& C(34 A)-C(33 A)-G a(2)-C(34 B) \\
& C(38 A)-C(33 A)-G a(2)-C(34 B) \\
& C(32 A)-C(33 A)-G a(2)-E u \\
& C(34 A)-C(33 A)-G a(2)-E u \\
& C(38 A)-C(33 A)-G a(2)-E u \\
& C(33 A)-C(32 A)-G a(2)-C(32 B) \\
& C(31 A)-C(32 A)-G a(2)-C(32 B) \\
& C(37 A)-C(32 A)-G a(2)-C(32 B) \\
& C(33 A)-C(32 A)-G a(2)-C(31 B) \\
& C(31 A)-C(32 A)-G a(2)-C(31 B) \\
& C(37 A)-C(32 A)-G a(2)-C(31 B) \\
& C(33 A)-C(32 A)-G a(2)-C(33 B) \\
& C(31 A)-C(32 A)-G a(2)-C(33 B) \\
& C(37 A)-C(32 A)-G a(2)-C(33 B) \\
& C(33 A)-C(32 A)-G a(2)-C(35 A) \\
& C(31 A)-C(32 A)-G a(2)-C(35 A) \\
& C(37 A)-C(32 A)-G a(2)-C(35 A) \\
& C(33 A)-C(32 A)-G a(2)-C(34 A) \\
& C(31 A)-C(32 A)-G a(2)-C(34 A) \\
& C(37 A)-C(32 A)-G a(2)-C(34 A) \\
& C(33 A)-C(32 A)-G a(2)-C(35 B) \\
& C(31 A)-C(32 A)-G a(2)-C(35 B) \\
& C(37 A)-C(32 A)-G a(2)-C(35 B) \\
& C(33 A)-C(32 A)-G a(2)-C(31 A) \\
& C(37 A)-C(32 A)-G a(2)-C(31 A) \\
& C(31 A)-C(32 A)-G a(2)-C(33 A) \\
& C(37 A)-C(32 A)-G a(2)-C(33 A) \\
& C(33 A)-C(32 A)-G a(2)-C(34 B) \\
& C(31 A)-C(32 A)-G a(2)-C(34 B) \\
& C(37 A)-C(32 A)-G a(2)-C(34 B) \\
& C(33 A)-C(32 A)-G a(2)-E u \\
& C(31 A)-C(32 A)-G a(2)-E u \\
& C(37 A)-C(32 A)-G a(2)-E u \\
& C(33 B)-C(34 B)-G a(2)-C(32 B)
\end{aligned}
$$

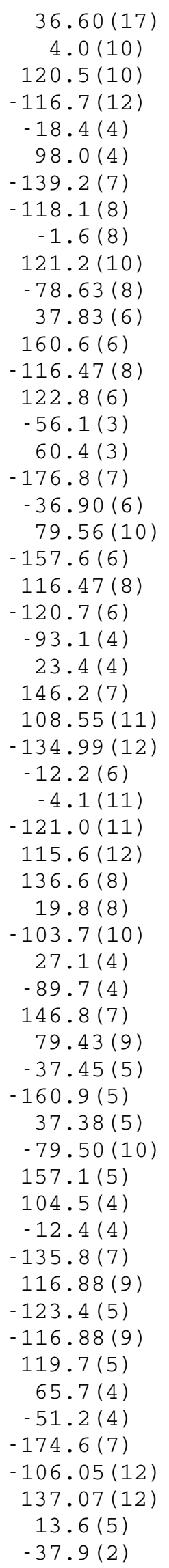




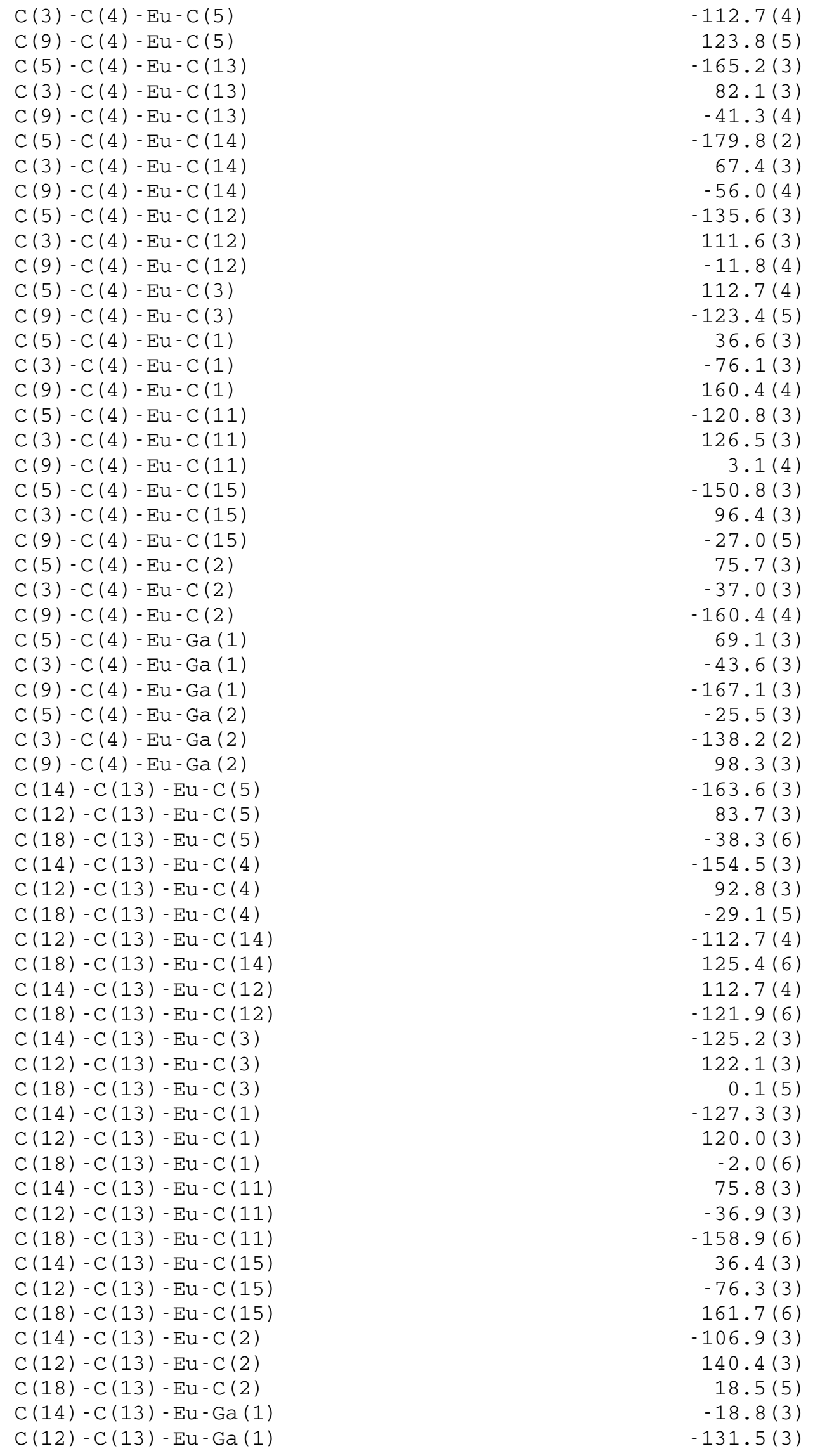




\begin{tabular}{|c|c|}
\hline $\mathrm{C}(18)-\mathrm{C}(13)-\mathrm{Eu}-\mathrm{Ga}(1)$ & $106.6(5)$ \\
\hline $\mathrm{C}(14)-\mathrm{C}(13)-\mathrm{Eu}-\mathrm{Ga}(2)$ & $74.9(3)$ \\
\hline $\mathrm{C}(12)-\mathrm{C}(13)-\mathrm{Eu}-\mathrm{Ga}(2)$ & $-37.8(3)$ \\
\hline $\mathrm{C}(18)-\mathrm{C}(13)-\mathrm{Eu}-\mathrm{Ga}(2)$ & $-159.7(5)$ \\
\hline$C(13)-C(14)-E u-C(5)$ & $31.0(5)$ \\
\hline$C(15)-C(14)-E u-C(5)$ & $145.3(3)$ \\
\hline$C(19)-C(14)-E u-C(5)$ & $-90.9(5)$ \\
\hline$C(13)-C(14)-E u-C(4)$ & $31.1(3)$ \\
\hline$C(15)-C(14)-E u-C(4)$ & $145.5(3)$ \\
\hline$C(19)-C(14)-E u-C(4)$ & $-90.7(5)$ \\
\hline$C(15)-C(14)-E u-C(13)$ & $114.3(4)$ \\
\hline$C(19)-C(14)-E u-C(13)$ & $-121.8(6)$ \\
\hline$C(13)-C(14)-E u-C(12)$ & $-37.4(3)$ \\
\hline$C(15)-C(14)-E u-C(12)$ & $76.9(3)$ \\
\hline$C(19)-C(14)-E u-C(12)$ & $-159.2(5)$ \\
\hline$C(13)-C(14)-E u-C(3)$ & $60.0(3)$ \\
\hline$C(15)-C(14)-E u-C(3)$ & $174.4(3)$ \\
\hline$C(19)-C(14)-E u-C(3)$ & $-61.8(5)$ \\
\hline$C(13)-C(14)-E u-C(1)$ & $97.0(4)$ \\
\hline$C(15)-C(14)-E u-C(1)$ & $-148.6(3)$ \\
\hline$C(19)-C(14)-E u-C(1)$ & $-24.8(6)$ \\
\hline$C(13)-C(14)-E u-C(11)$ & $-77.3(3)$ \\
\hline$C(15)-C(14)-E u-C(11)$ & $37.1(3)$ \\
\hline$C(19)-C(14)-E u-C(11)$ & $160.9(5)$ \\
\hline$C(13)-C(14)-E u-C(15)$ & $-114.3(4)$ \\
\hline$C(19)-C(14)-E u-C(15)$ & $123.8(6)$ \\
\hline$C(13)-C(14)-E u-C(2)$ & $89.9(3)$ \\
\hline$C(15)-C(14)-E u-C(2)$ & $-155.8(3)$ \\
\hline$C(19)-C(14)-E u-C(2)$ & $-31.9(5)$ \\
\hline $\mathrm{C}(13)-\mathrm{C}(14)-\mathrm{Eu}-\mathrm{Ga}(1)$ & $162.5(3)$ \\
\hline $\mathrm{C}(15)-\mathrm{C}(14)-\mathrm{Eu}-\mathrm{Ga}(1)$ & $-83.2(3)$ \\
\hline C (19) - C (14)-Eu-Ga (1) & $40.7(4)$ \\
\hline $\mathrm{C}(13)-\mathrm{C}(14)-\mathrm{Eu}-\mathrm{Ga}(2)$ & $-122.5(3)$ \\
\hline $\mathrm{C}(15)-\mathrm{C}(14)-\mathrm{Eu}-\mathrm{Ga}(2)$ & $-8.2(3)$ \\
\hline $\mathrm{C}(19)-\mathrm{C}(14)-\mathrm{Eu}-\mathrm{Ga}(2)$ & $115.6(4)$ \\
\hline$C(13)-C(12)-E u-C(5)$ & $-115.2(3)$ \\
\hline$C(11)-C(12)-E u-C(5)$ & $131.3(3)$ \\
\hline$C(17)-C(12)-E u-C(5)$ & $8.0(6)$ \\
\hline$C(13)-C(12)-E u-C(4)$ & $-91.9(3)$ \\
\hline$C(11)-C(12)-E u-C(4)$ & $154.5(3)$ \\
\hline$C(17)-C(12)-E u-C(4)$ & $31.2(5)$ \\
\hline$C(11)-C(12)-E u-C(13)$ & $-113.5(4)$ \\
\hline$C(17)-C(12)-E u-C(13)$ & $123.2(7)$ \\
\hline$C(13)-C(12)-E u-C(14)$ & $37.2(3)$ \\
\hline$C(11)-C(12)-E u-C(14)$ & $-76.4(3)$ \\
\hline$C(17)-C(12)-E u-C(14)$ & $160.3(6)$ \\
\hline$C(13)-C(12)-E u-C(3)$ & $-63.1(3)$ \\
\hline$C(11)-C(12)-E u-C(3)$ & $-176.6(3)$ \\
\hline$C(17)-C(12)-E u-C(3)$ & $60.1(5)$ \\
\hline$C(13)-C(12)-E u-C(1)$ & $-102.7(3)$ \\
\hline$C(11)-C(12)-E u-C(1)$ & $143.7(3)$ \\
\hline$C(17)-C(12)-E u-C(1)$ & $20.4(7)$ \\
\hline$C(13)-C(12)-E u-C(11)$ & $113.5(4)$ \\
\hline$C(17)-C(12)-E u-C(11)$ & $-123.3(6)$ \\
\hline$C(13)-C(12)-E u-C(15)$ & $76.5(3)$ \\
\hline$C(11)-C(12)-E u-C(15)$ & $-37.0(3)$ \\
\hline$C(17)-C(12)-E u-C(15)$ & $-160.3(6)$ \\
\hline$C(13)-C(12)-E u-C(2)$ & $-56.7(3)$ \\
\hline
\end{tabular}




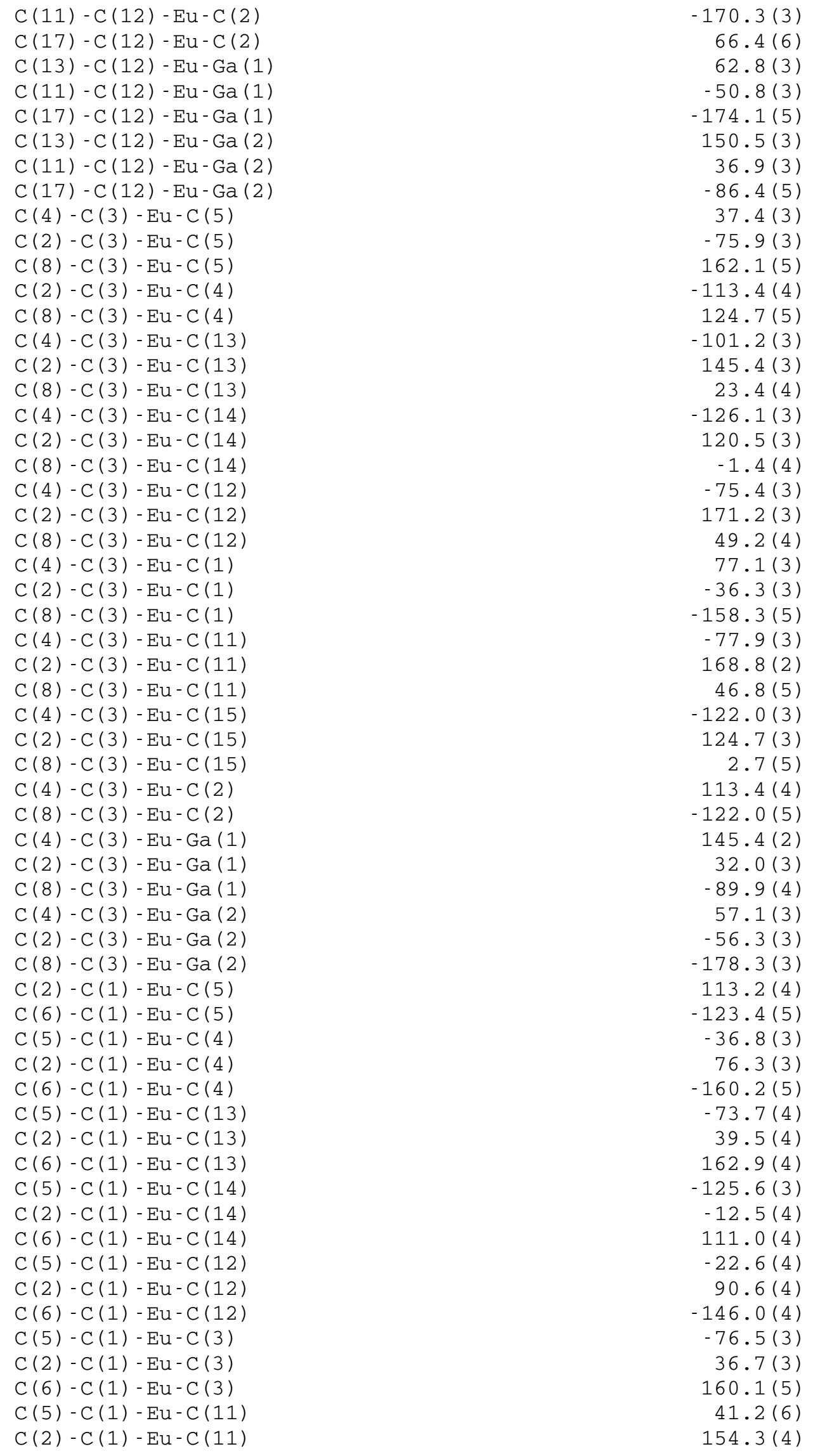




\begin{tabular}{|c|c|}
\hline$C(6)-C(1)-E u-C(11)$ & $-82.2(6)$ \\
\hline$C(5)-C(1)-E u-C(15)$ & $159.5(5)$ \\
\hline$C(2)-C(1)-E u-C(15)$ & $-87.4(6)$ \\
\hline$C(6)-C(1)-E u-C(15)$ & $36.1(8)$ \\
\hline$C(5)-C(1)-E u-C(2)$ & $-113.2(4)$ \\
\hline$C(6)-C(1)-E u-C(2)$ & $123.5(5)$ \\
\hline$C(5)-C(1)-E u-G a(1)$ & $168.8(3)$ \\
\hline$C(2)-C(1)-E u-G a(1)$ & $-78.0(3)$ \\
\hline$C(6)-C(1)-E u-G a(1)$ & $45.4(4)$ \\
\hline$C(5)-C(1)-E u-G a(2)$ & $89.1(3)$ \\
\hline$C(2)-C(1)-E u-G a(2)$ & $-157.7(3)$ \\
\hline$C(6)-C(1)-E u-G a(2)$ & $-34.3(4)$ \\
\hline$C(15)-C(11)-E u-C(5)$ & $178.9(2)$ \\
\hline$C(12)-C(11)-E u-C(5)$ & $-68.0(3)$ \\
\hline$C(16)-C(11)-E u-C(5)$ & $54.7(6)$ \\
\hline$C(15)-C(11)-E u-C(4)$ & $-144.3(3)$ \\
\hline$C(12)-C(11)-E u-C(4)$ & $-31.2(3)$ \\
\hline$C(16)-C(11)-E u-C(4)$ & $91.4(6)$ \\
\hline$C(15)-C(11)-E u-C(13)$ & $-76.2(3)$ \\
\hline$C(12)-C(11)-E u-C(13)$ & $36.8(3)$ \\
\hline$C(16)-C(11)-E u-C(13)$ & $159.5(6)$ \\
\hline$C(15)-C(11)-E u-C(14)$ & $-36.9(3)$ \\
\hline$C(12)-C(11)-E u-C(14)$ & $76.2(3)$ \\
\hline$C(16)-C(11)-E u-C(14)$ & $-161.1(6)$ \\
\hline$C(15)-C(11)-E u-C(12)$ & $-113.1(4)$ \\
\hline$C(16)-C(11)-E u-C(12)$ & $122.7(7)$ \\
\hline$C(15)-C(11)-E u-C(3)$ & $-108.3(3)$ \\
\hline$C(12)-C(11)-E u-C(3)$ & $4.8(4)$ \\
\hline$C(16)-C(11)-E u-C(3)$ & $127.4(5)$ \\
\hline$C(15)-C(11)-E u-C(1)$ & $151.8(4)$ \\
\hline$C(12)-C(11)-E u-C(1)$ & $-95.1(5)$ \\
\hline$C(16)-C(11)-E u-C(1)$ & $27.6(8)$ \\
\hline$C(12)-C(11)-E u-C(15)$ & $113.1(4)$ \\
\hline$C(16)-C(11)-E u-C(15)$ & $-124.2(7)$ \\
\hline$C(15)-C(11)-E u-C(2)$ & $-85.8(6)$ \\
\hline$C(12)-C(11)-E u-C(2)$ & $27.3(7)$ \\
\hline$C(16)-C(11)-E u-C(2)$ & $149.9(6)$ \\
\hline $\mathrm{C}(15)-\mathrm{C}(11)-\mathrm{Eu}-\mathrm{Ga}(1)$ & $27.4(3)$ \\
\hline $\mathrm{C}(12)-\mathrm{C}(11)-\mathrm{Eu}-\mathrm{Ga}(1)$ & $140.5(3)$ \\
\hline $\mathrm{C}(16)-\mathrm{C}(11)-\mathrm{Eu}-\mathrm{Ga}(1)$ & $-96.9(5)$ \\
\hline $\mathrm{C}(15)-\mathrm{C}(11)-\mathrm{Eu}-\mathrm{Ga}(2)$ & $103.1(3)$ \\
\hline $\mathrm{C}(12)-\mathrm{C}(11)-\mathrm{Eu}-\mathrm{Ga}(2)$ & $-143.8(3)$ \\
\hline$C(16)-C(11)-E u-G a(2)$ & $-21.2(5)$ \\
\hline$C(14)-C(15)-E u-C(5)$ & $-116.0(5)$ \\
\hline$C(11)-C(15)-E u-C(5)$ & $-2.7(6)$ \\
\hline$C(20)-C(15)-E u-C(5)$ & $119.2(6)$ \\
\hline$C(14)-C(15)-E u-C(4)$ & $-55.6(4)$ \\
\hline$C(11)-C(15)-E u-C(4)$ & $57.6(4)$ \\
\hline$C(20)-C(15)-E u-C(4)$ & $179.6(4)$ \\
\hline$C(14)-C(15)-E u-C(13)$ & $-36.4(3)$ \\
\hline$C(11)-C(15)-E u-C(13)$ & $76.8(3)$ \\
\hline$C(20)-C(15)-E u-C(13)$ & $-161.2(6)$ \\
\hline$C(11)-C(15)-E u-C(14)$ & $113.3(4)$ \\
\hline$C(20)-C(15)-E u-C(14)$ & $-124.8(6)$ \\
\hline$C(14)-C(15)-E u-C(12)$ & $-76.0(3)$ \\
\hline$C(11)-C(15)-E u-C(12)$ & $37.2(3)$ \\
\hline$C(20)-C(15)-E u-C(12)$ & $159.2(6)$ \\
\hline$C(14)-C(15)-E u-C(3)$ & $-8.0(4)$ \\
\hline
\end{tabular}




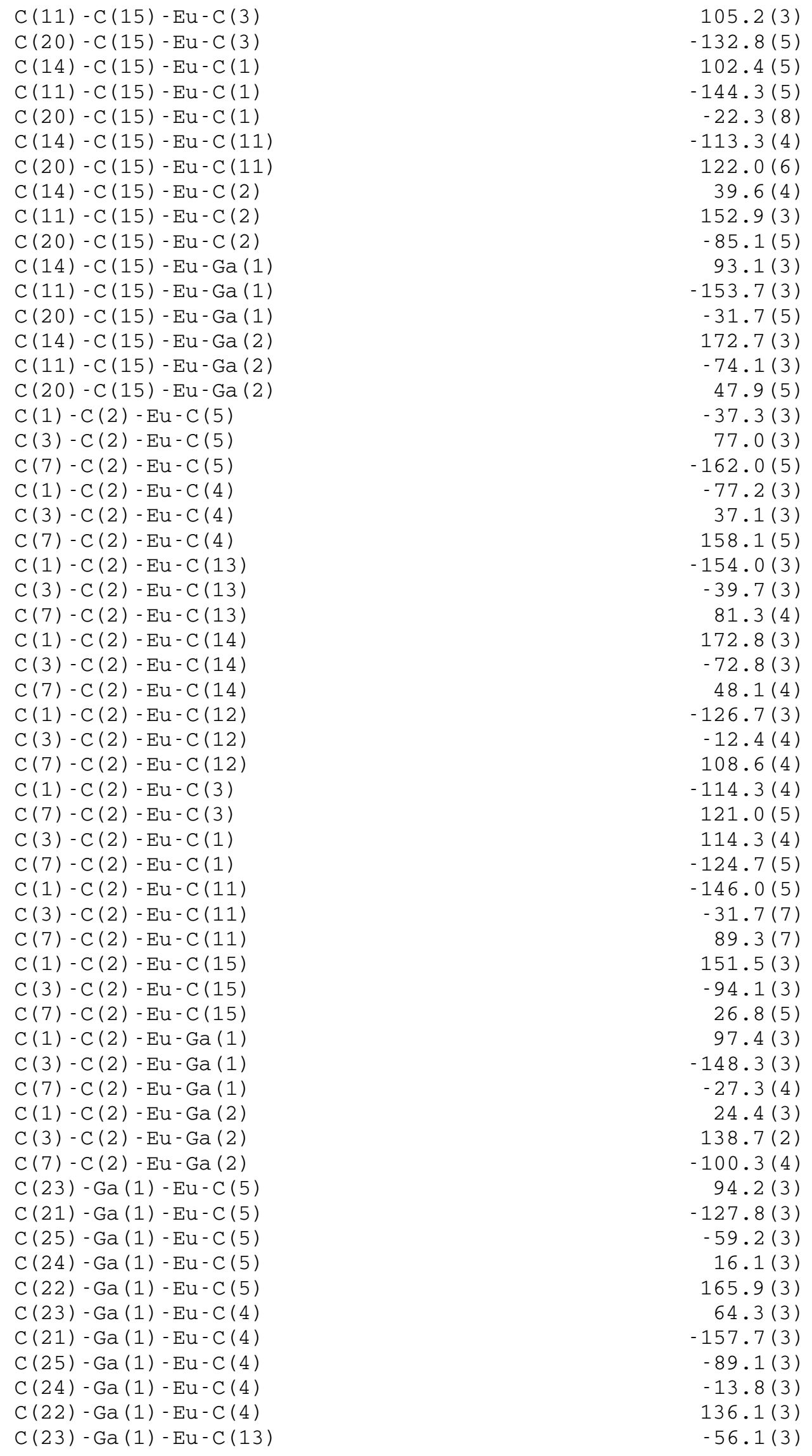




\begin{tabular}{|c|c|}
\hline $\mathrm{C}(21)-\mathrm{Ga}(1)-\mathrm{Eu}-\mathrm{C}(13)$ & $81.9(3)$ \\
\hline $\mathrm{C}(25)-\mathrm{Ga}(1)-\mathrm{Eu}-\mathrm{C}(13)$ & $150.5(3)$ \\
\hline $\mathrm{C}(24)-\mathrm{Ga}(1)-\mathrm{Eu}-\mathrm{C}(13)$ & $-134.2(3)$ \\
\hline $\mathrm{C}(22)-\mathrm{Ga}(1)-\mathrm{Eu}-\mathrm{C}(13)$ & $15.7(3)$ \\
\hline $\mathrm{C}(23)-\mathrm{Ga}(1)-\mathrm{Eu}-\mathrm{C}(14)$ & $-65.1(3)$ \\
\hline $\mathrm{C}(21)-\mathrm{Ga}(1)-\mathrm{Eu}-\mathrm{C}(14)$ & $72.9(3)$ \\
\hline$C(25)-\mathrm{Ga}(1)-\mathrm{Eu}-\mathrm{C}(14)$ & $141.5(3)$ \\
\hline $\mathrm{C}(24)-\mathrm{Ga}(1)-\mathrm{Eu}-\mathrm{C}(14)$ & $-143.2(3)$ \\
\hline C (22)-Ga (1)-Eu-C (14) & $6.6(3)$ \\
\hline $\mathrm{C}(23)-\mathrm{Ga}(1)-\mathrm{Eu}-\mathrm{C}(12)$ & $-83.9(3)$ \\
\hline $\mathrm{C}(21)-\mathrm{Ga}(1)-\mathrm{Eu}-\mathrm{C}(12)$ & $54.2(3)$ \\
\hline $\mathrm{C}(25)-\mathrm{Ga}(1)-\mathrm{Eu}-\mathrm{C}(12)$ & $122.8(3)$ \\
\hline $\mathrm{C}(24)-\mathrm{Ga}(1)-\mathrm{Eu}-\mathrm{C}(12)$ & $-161.9(3)$ \\
\hline $\mathrm{C}(22)-\mathrm{Ga}(1)-\mathrm{Eu}-\mathrm{C}(12)$ & $-12.1(3)$ \\
\hline $\mathrm{C}(23)-\mathrm{Ga}(1)-\mathrm{Eu}-\mathrm{C}(3)$ & $43.8(3)$ \\
\hline $\mathrm{C}(21)-\mathrm{Ga}(1)-\mathrm{Eu}-\mathrm{C}(3)$ & $-178.1(3)$ \\
\hline $\mathrm{C}(25)-\mathrm{Ga}(1)-\mathrm{Eu}-\mathrm{C}(3)$ & $-109.5(3)$ \\
\hline $\mathrm{C}(24)-\mathrm{Ga}(1)-\mathrm{Eu}-\mathrm{C}(3)$ & $-34.2(3)$ \\
\hline $\mathrm{C}(22)-\mathrm{Ga}(1)-\mathrm{Eu}-\mathrm{C}(3)$ & $115.6(3)$ \\
\hline $\mathrm{C}(23)-\mathrm{Ga}(1)-\mathrm{Eu}-\mathrm{C}(1)$ & $88.3(3)$ \\
\hline $\mathrm{C}(21)-\mathrm{Ga}(1)-\mathrm{Eu}-\mathrm{C}(1)$ & $-133.7(3)$ \\
\hline $\mathrm{C}(25)-\mathrm{Ga}(1)-\mathrm{Eu}-\mathrm{C}(1)$ & $-65.1(3)$ \\
\hline $\mathrm{C}(24)-\mathrm{Ga}(1)-\mathrm{Eu}-\mathrm{C}(1)$ & $10.2(3)$ \\
\hline $\mathrm{C}(22)-\mathrm{Ga}(1)-\mathrm{Eu}-\mathrm{C}(1)$ & $160.0(3)$ \\
\hline $\mathrm{C}(23)-\mathrm{Ga}(1)-\mathrm{Eu}-\mathrm{C}(11)$ & $-107.2(3)$ \\
\hline $\mathrm{C}(21)-\mathrm{Ga}(1)-\mathrm{Eu}-\mathrm{C}(11)$ & $30.8(3)$ \\
\hline $\mathrm{C}(25)-\mathrm{Ga}(1)-\mathrm{Eu}-\mathrm{C}(11)$ & $99.4(3)$ \\
\hline $\mathrm{C}(24)-\mathrm{Ga}(1)-\mathrm{Eu}-\mathrm{C}(11)$ & $174.7(3)$ \\
\hline $\mathrm{C}(22)-\mathrm{Ga}(1)-\mathrm{Eu}-\mathrm{C}(11)$ & $-35.5(3)$ \\
\hline $\mathrm{C}(23)-\mathrm{Ga}(1)-\mathrm{Eu}-\mathrm{C}(15)$ & $-94.2(3)$ \\
\hline $\mathrm{C}(21)-\mathrm{Ga}(1)-\mathrm{Eu}-\mathrm{C}(15)$ & $43.9(3)$ \\
\hline$C(25)-\mathrm{Ga}(1)-\mathrm{Eu}-\mathrm{C}(15)$ & $112.5(3)$ \\
\hline $\mathrm{C}(24)-\mathrm{Ga}(1)-\mathrm{Eu}-\mathrm{C}(15)$ & $-172.2(3)$ \\
\hline $\mathrm{C}(22)-\mathrm{Ga}(1)-\mathrm{Eu}-\mathrm{C}(15)$ & $-22.4(3)$ \\
\hline $\mathrm{C}(23)-\mathrm{Ga}(1)-\mathrm{Eu}-\mathrm{C}(2)$ & $59.2(3)$ \\
\hline $\mathrm{C}(21)-\mathrm{Ga}(1)-\mathrm{Eu}-\mathrm{C}(2)$ & $-162.7(3)$ \\
\hline $\mathrm{C}(25)-\mathrm{Ga}(1)-\mathrm{Eu}-\mathrm{C}(2)$ & $-94.1(3)$ \\
\hline $\mathrm{C}(24)-\mathrm{Ga}(1)-\mathrm{Eu}-\mathrm{C}(2)$ & $-18.8(3)$ \\
\hline $\mathrm{C}(22)-\mathrm{Ga}(1)-\mathrm{Eu}-\mathrm{C}(2)$ & $131.0(3)$ \\
\hline $\mathrm{C}(23)-\mathrm{Ga}(1)-\mathrm{Eu}-\mathrm{Ga}(2)$ & $176.2(3)$ \\
\hline $\mathrm{C}(21)-\mathrm{Ga}(1)-\mathrm{Eu}-\mathrm{Ga}(2)$ & $-45.7(3)$ \\
\hline $\mathrm{C}(25)-\mathrm{Ga}(1)-\mathrm{Eu}-\mathrm{Ga}(2)$ & $22.9(3)$ \\
\hline C (24)-Ga (1)-Eu-Ga (2) & $98.2(3)$ \\
\hline $\mathrm{C}(22)-\mathrm{Ga}(1)-\mathrm{Eu}-\mathrm{Ga}(2)$ & $-112.0(3)$ \\
\hline$C(32 B)-G a(2)-E u-C(5)$ & $-59.9(5)$ \\
\hline$C(31 B)-G a(2)-E u-C(5)$ & $-5.4(5)$ \\
\hline$C(33 B)-G a(2)-E u-C(5)$ & $-115.0(6)$ \\
\hline$C(35 A)-G a(2)-E u-C(5)$ & $123.4(5)$ \\
\hline$C(34 A)-G a(2)-E u-C(5)$ & $-136.7(2)$ \\
\hline$C(35 B)-G a(2)-E u-C(5)$ & $58.1(8)$ \\
\hline$C(31 A)-G a(2)-E u-C(5)$ & $16.8(2)$ \\
\hline$C(33 A)-G a(2)-E u-C(5)$ & $-84.52(18)$ \\
\hline$C(32 A)-G a(2)-E u-C(5)$ & $-35.66(18)$ \\
\hline$C(34 B)-G a(2)-E u-C(5)$ & $168.4(11)$ \\
\hline$C(32 B)-G a(2)-E u-C(4)$ & $-47.6(5)$ \\
\hline$C(31 B)-G a(2)-E u-C(4)$ & $6.9(5)$ \\
\hline$C(33 B)-G a(2)-E u-C(4)$ & $-102.7(6)$ \\
\hline$C(35 A)-G a(2)-E u-C(4)$ & $135.7(5)$ \\
\hline
\end{tabular}




\begin{tabular}{|c|c|}
\hline$C(34 A)-G a(2)-E u-C(4)$ & $-124.4(2)$ \\
\hline$C(35 B)-G a(2)-E u-C(4)$ & $70.4(8)$ \\
\hline$C(31 A)-G a(2)-E u-C(4)$ & $29.1(2)$ \\
\hline$C(33 A)-G a(2)-E u-C(4)$ & $-72.24(18)$ \\
\hline$C(32 A)-G a(2)-E u-C(4)$ & $-23.39(17)$ \\
\hline$C(34 B)-G a(2)-E u-C(4)$ & $-179.3(11)$ \\
\hline$C(32 B)-G a(2)-E u-C(13)$ & $77.3(5)$ \\
\hline$C(31 B)-G a(2)-E u-C(13)$ & $131.8(5)$ \\
\hline$C(33 B)-G a(2)-E u-C(13)$ & $22.2(6)$ \\
\hline$C(35 A)-G a(2)-E u-C(13)$ & $-99.4(5)$ \\
\hline$C(34 A)-G a(2)-E u-C(13)$ & $0.5(2)$ \\
\hline$C(35 B)-G a(2)-E u-C(13)$ & $-164.7(8)$ \\
\hline$C(31 A)-G a(2)-E u-C(13)$ & $154.0(2)$ \\
\hline$C(33 A)-G a(2)-E u-C(13)$ & $52.7(2)$ \\
\hline$C(32 A)-G a(2)-E u-C(13)$ & $101.52(19)$ \\
\hline$C(34 B)-G a(2)-E u-C(13)$ & $-54.4(11)$ \\
\hline$C(32 B)-G a(2)-E u-C(14)$ & $108.9(5)$ \\
\hline$C(31 B)-G a(2)-E u-C(14)$ & $163.3(5)$ \\
\hline$C(33 B)-G a(2)-E u-C(14)$ & $53.8(6)$ \\
\hline$C(35 A)-G a(2)-E u-C(14)$ & $-67.8(5)$ \\
\hline$C(34 A)-G a(2)-E u-C(14)$ & $32.0(2)$ \\
\hline$C(35 B)-G a(2)-E u-C(14)$ & $-133.1(8)$ \\
\hline$C(31 A)-G a(2)-E u-C(14)$ & $-174.5(2)$ \\
\hline$C(33 A)-G a(2)-E u-C(14)$ & $84.24(18)$ \\
\hline$C(32 A)-G a(2)-E u-C(14)$ & $133.10(18)$ \\
\hline$C(34 B)-G a(2)-E u-C(14)$ & $-22.8(11)$ \\
\hline$C(32 B)-G a(2)-E u-C(12)$ & $59.4(5)$ \\
\hline$C(31 B)-G a(2)-E u-C(12)$ & $113.8(5)$ \\
\hline$C(33 B)-G a(2)-E u-C(12)$ & $4.3(6)$ \\
\hline$C(35 A)-G a(2)-E u-C(12)$ & $-117.3(5)$ \\
\hline$C(34 A)-G a(2)-E u-C(12)$ & $-17.5(2)$ \\
\hline$C(35 B)-G a(2)-E u-C(12)$ & $177.4(8)$ \\
\hline$C(31 A)-G a(2)-E u-C(12)$ & $136.0(2)$ \\
\hline$C(33 A)-G a(2)-E u-C(12)$ & $34.74(17)$ \\
\hline$C(32 A)-G a(2)-E u-C(12)$ & $83.59(17)$ \\
\hline$C(34 B)-G a(2)-E u-C(12)$ & $-72.3(11)$ \\
\hline$C(32 B)-G a(2)-E u-C(3)$ & $-74.5(5)$ \\
\hline$C(31 B)-G a(2)-E u-C(3)$ & $-20.0(5)$ \\
\hline$C(33 B)-G a(2)-E u-C(3)$ & $-129.5(6)$ \\
\hline$C(35 A)-G a(2)-E u-C(3)$ & $108.9(5)$ \\
\hline$C(34 A)-G a(2)-E u-C(3)$ & $-151.3(2)$ \\
\hline$C(35 B)-G a(2)-E u-C(3)$ & $43.5(8)$ \\
\hline$C(31 A)-G a(2)-E u-C(3)$ & $2.2(2)$ \\
\hline$C(33 A)-G a(2)-E u-C(3)$ & $-99.1(2)$ \\
\hline$C(32 A)-G a(2)-E u-C(3)$ & $-50.2(2)$ \\
\hline$C(34 B)-G a(2)-E u-C(3)$ & $153.8(11)$ \\
\hline$C(32 B)-G a(2)-E u-C(1)$ & $-89.3(5)$ \\
\hline$C(31 B)-G a(2)-E u-C(1)$ & $-34.9(5)$ \\
\hline$C(33 B)-G a(2)-E u-C(1)$ & $-144.4(6)$ \\
\hline$C(35 A)-G a(2)-E u-C(1)$ & $94.0(5)$ \\
\hline$C(34 A)-G a(2)-E u-C(1)$ & $-166.1(2)$ \\
\hline$C(35 B)-G a(2)-E u-C(1)$ & $28.7(8)$ \\
\hline$C(31 A)-G a(2)-E u-C(1)$ & $-12.6(2)$ \\
\hline$C(33 A)-G a(2)-E u-C(1)$ & $-113.95(18)$ \\
\hline$C(32 A)-G a(2)-E u-C(1)$ & $-65.09(17)$ \\
\hline$C(34 B)-G a(2)-E u-C(1)$ & $139.0(11)$ \\
\hline$C(32 B)-G a(2)-E u-C(11)$ & $76.6(5)$ \\
\hline$C(31 B)-G a(2)-E u-C(11)$ & $131.1(5)$ \\
\hline
\end{tabular}




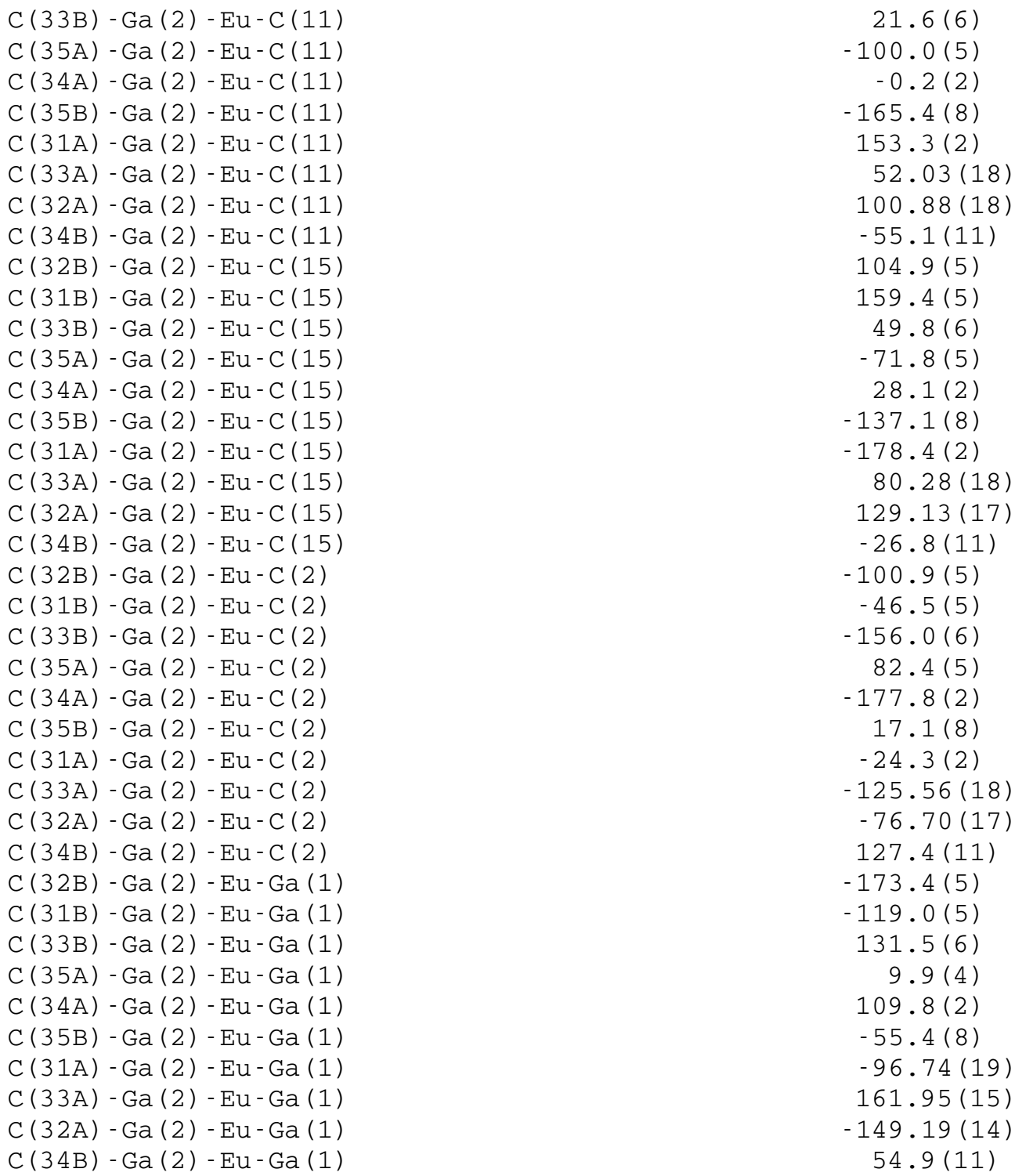

Symmetry transformations used to generate equivalent atoms: 
Perspective ORTEP view of the molecular structure of 2. Thermal ellipsoids are drawn to encompass $50 \%$ probability. Hydrogen atoms are omitted for clarity.

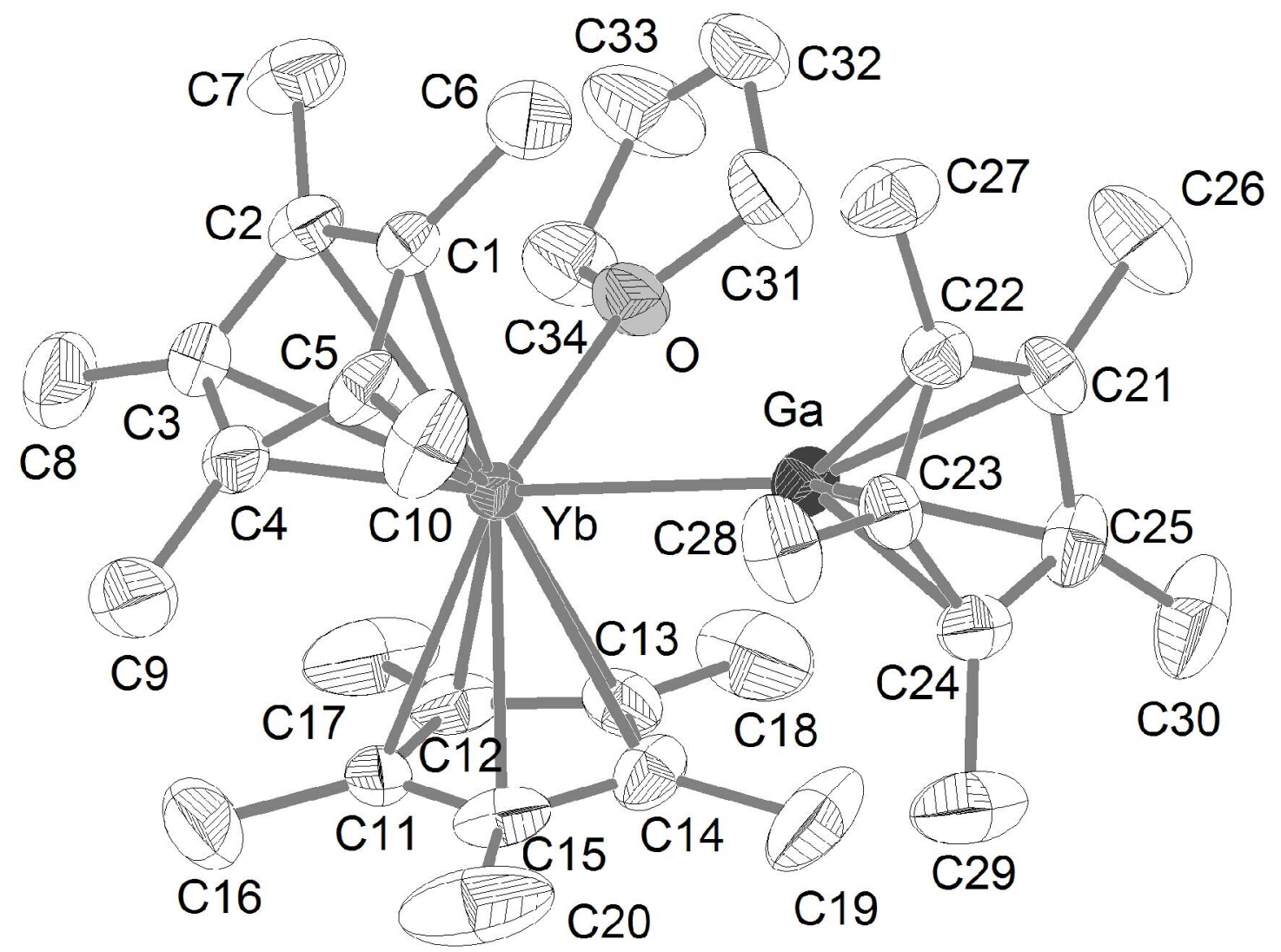


Table 1. Crystal data and structure refinement for 2 .

\begin{tabular}{|c|c|}
\hline Identification code & mw2 7 \\
\hline Empirical formula & $\mathrm{C} 34 \mathrm{H} 53 \mathrm{Ga} O \mathrm{Yb}$ \\
\hline Formula weight & 720.52 \\
\hline Temperature & $200(2) \mathrm{K}$ \\
\hline Wavelength & $0.71073 \mathrm{~A}$ \\
\hline Crystal system, space group & Orthorhombic, $\quad \mathrm{P}$ b $\mathrm{c}$ a \\
\hline Unit cell dimensions & $\begin{aligned} \mathrm{a} & =15.9742(7) \mathrm{A} & \text { alpha } & =90 \text { deg. } \\
\mathrm{b} & =22.7462(11) \mathrm{A} & \text { beta } & =90 \mathrm{deg} . \\
\mathrm{c} & =18.2842(13) \mathrm{A} & \text { gamma } & =90 \mathrm{deg} .\end{aligned}$ \\
\hline Volume & $6643.6(6) \quad A \wedge 3$ \\
\hline Z, Calculated density & 8, $\quad 1.441 \mathrm{Mg} / \mathrm{m} \wedge 3$ \\
\hline Absorption coefficient & $3.631 \mathrm{~mm} \wedge-1$ \\
\hline$F(000)$ & 2928 \\
\hline Crystal size & $0.391 \times 0.326 \times 0.274 \mathrm{~mm}$ \\
\hline Theta range for data collection & 2.70 to $25.02 \mathrm{deg}$. \\
\hline Limiting indices & $-18<=h<=16, \quad-23<=\mathrm{k}<=27, \quad-17<=1<=21$ \\
\hline Reflections collected / unique & $16462 / 5848[R($ int $)=0.0380]$ \\
\hline Completeness to theta $=25.02$ & $99.8 \%$ \\
\hline Absorption correction & Integration \\
\hline Max. and min. transmission & 0.4177 and 0.2662 \\
\hline Refinement method & Full-matrix least-squares on $F^{\wedge} 2$ \\
\hline Data / restraints / parameters & $5848 / 0 / 349$ \\
\hline Goodness-of-fit on $F^{\wedge} 2$ & 1.004 \\
\hline Final $R$ indices [I>2sigma(I)] & $\mathrm{R} 1=0.0259, \mathrm{wR} 2=0.0469$ \\
\hline $\mathrm{R}$ indices (all data) & $\mathrm{R} 1=0.0425, \mathrm{wR} 2=0.0496$ \\
\hline
\end{tabular}


Table 2. Atomic coordinates $(\mathrm{x} 10 \wedge 4)$ and equivalent isotropic displacement parameters $(A \wedge 2 \times 10 \wedge 3)$ for 2 .

$\mathrm{U}(\mathrm{eq})$ is defined as one third of the trace of the orthogonalized Uij tensor.

\begin{tabular}{|c|c|c|c|c|}
\hline & $\mathrm{x}$ & y & z & $\mathrm{U}(\mathrm{eq})$ \\
\hline$C(1)$ & $1923(3)$ & $9330(2)$ & $-262(2)$ & $36(1)$ \\
\hline$C(2)$ & $1378(2)$ & $8848(2)$ & $-265(2)$ & $38(1)$ \\
\hline$C(3)$ & $1865(2)$ & $8327(2)$ & $-279(2)$ & $37(1)$ \\
\hline$C(4)$ & $2711(2)$ & $8496(2)$ & $-285(2)$ & $36(1)$ \\
\hline$C(5)$ & $2752(2)$ & $9117(2)$ & $-271(2)$ & $31(1)$ \\
\hline$C(6)$ & $1665(3)$ & $9964(2)$ & $-340(2)$ & $59(1)$ \\
\hline$C(7)$ & $447(3)$ & $8878(2)$ & $-390(2)$ & $61(1)$ \\
\hline$C(8)$ & $1548(3)$ & $7706(2)$ & $-364(3)$ & $61(1)$ \\
\hline$C(9)$ & $3454(3)$ & $8095(2)$ & $-410(3)$ & $57(1)$ \\
\hline$C(10)$ & $3537(3)$ & $9478(2)$ & $-344(2)$ & $51(1)$ \\
\hline$C(11)$ & $707(3)$ & $8974(2)$ & $1852(2)$ & $43(1)$ \\
\hline$C(12)$ & $1350(3)$ & $9061(2)$ & $2348(2)$ & $39(1)$ \\
\hline$C(13)$ & $1747(2)$ & $8522(2)$ & $2475(2)$ & $38(1)$ \\
\hline$C(14)$ & $1346(3)$ & $8094(2)$ & $2047(2)$ & $41(1)$ \\
\hline$C(15)$ & $691(2)$ & $8371(2)$ & $1659(2)$ & $45(1)$ \\
\hline$C(16)$ & $87(4)$ & $9433(3)$ & $1597(3)$ & $92(2)$ \\
\hline$C(17)$ & $1499(4)$ & $9613(2)$ & $2794(3)$ & $82(2)$ \\
\hline$C(18)$ & $2419(3)$ & $8424(3)$ & $3046(3)$ & $78(2)$ \\
\hline$C(19)$ & $1504(4)$ & $7439(2)$ & $2062(3)$ & $84(2)$ \\
\hline$C(20)$ & $29(3)$ & $8053(3)$ & $1230(3)$ & $94(2)$ \\
\hline$C(21)$ & $3671(2)$ & $10903(2)$ & $1620(2)$ & $38(1)$ \\
\hline$C(22)$ & $3331(2)$ & $11042(2)$ & $923(2)$ & $33(1)$ \\
\hline$C(23)$ & $2471(2)$ & $11175(1)$ & $1022(2)$ & $32(1)$ \\
\hline$C(24)$ & $2284(2)$ & $11112(2)$ & $1783(2)$ & $37(1)$ \\
\hline$C(25)$ & $3034(3)$ & $10954(2)$ & $2143(2)$ & $41(1)$ \\
\hline$C(26)$ & $4577(3)$ & $10779(2)$ & $1772(3)$ & $75(2)$ \\
\hline$C(27)$ & $3816(3)$ & $11092(2)$ & $229(2)$ & $55(1)$ \\
\hline$C(28)$ & $1881(3)$ & $11390(2)$ & $439(3)$ & $55(1)$ \\
\hline C (29) & $1460(3)$ & $11252(2)$ & $2133(3)$ & $70(2)$ \\
\hline$C(30)$ & $3128(4)$ & $10885(2)$ & $2957(2)$ & $73(2)$ \\
\hline$C(31)$ & $4248(3)$ & $8800(2)$ & $1559(3)$ & $63(1)$ \\
\hline C (32) & $4931(3)$ & $8375(2)$ & $1388(3)$ & $59(1)$ \\
\hline$C(33)$ & $4562(3)$ & $7798(2)$ & $1555(4)$ & $81(2)$ \\
\hline C (34) & $3653(3)$ & $7874(2)$ & $1563(3)$ & $56(1)$ \\
\hline$O(1)$ & $3483(2)$ & $8487(1)$ & $1441(2)$ & $42(1)$ \\
\hline $\mathrm{Ga}(1)$ & $2675(1)$ & $10182(1)$ & $1360(1)$ & $34(1)$ \\
\hline $\mathrm{Yb}(1)$ & $2111(1)$ & $8814(1)$ & $1059(1)$ & $26(1)$ \\
\hline
\end{tabular}


Table 3. Bond lengths [A] and angles [deg] for 2 .

\begin{tabular}{|c|c|}
\hline$C(1)-C(2)$ & $1.401(6)$ \\
\hline$C(1)-C(5)$ & $1.410(5)$ \\
\hline$C(1)-C(6)$ & $1.507(5)$ \\
\hline$C(1)-Y b(1)$ & $2.701(4)$ \\
\hline$C(2)-C(3)$ & $1.417(5)$ \\
\hline$C(2)-C(7)$ & $1.507(5)$ \\
\hline$C(2)-Y b(1)$ & $2.690(3)$ \\
\hline$C(3)-C(4)$ & $1.404(5)$ \\
\hline$C(3)-C(8)$ & $1.509(6)$ \\
\hline$C(3)-Y b(1)$ & $2.714(4)$ \\
\hline$C(4)-C(5)$ & $1.414(5)$ \\
\hline$C(4)-C(9)$ & $1.514(5)$ \\
\hline$C(4)-Y b(1)$ & $2.735(4)$ \\
\hline$C(5)-C(10)$ & $1.506(5)$ \\
\hline$C(5)-Y b(1)$ & $2.726(3)$ \\
\hline$C(11)-C(12)$ & $1.385(6)$ \\
\hline$C(11)-C(15)$ & $1.417(6)$ \\
\hline$C(11)-C(16)$ & $1.513(6)$ \\
\hline $\mathrm{C}(11)-\mathrm{Yb}(1)$ & $2.696(4)$ \\
\hline$C(12)-C(13)$ & $1.400(5)$ \\
\hline$C(12)-C(17)$ & $1.515(6)$ \\
\hline$C(12)-Y b(1)$ & $2.711(4)$ \\
\hline$C(13)-C(14)$ & $1.405(6)$ \\
\hline$C(13)-C(18)$ & $1.514(6)$ \\
\hline$C(13)-Y b(1)$ & $2.735(4)$ \\
\hline$C(14)-C(15)$ & $1.412(6)$ \\
\hline$C(14)-C(19)$ & $1.510(5)$ \\
\hline $\mathrm{C}(14)-\mathrm{Yb}(1)$ & $2.728(4)$ \\
\hline$C(15)-C(20)$ & $1.502(6)$ \\
\hline$C(15)-Y b(1)$ & $2.713(4)$ \\
\hline$C(21)-C(25)$ & $1.401(6)$ \\
\hline$C(21)-C(22)$ & $1.422(5)$ \\
\hline$C(21)-C(26)$ & $1.502(6)$ \\
\hline $\mathrm{C}(21)-\mathrm{Ga}(1)$ & $2.333(4)$ \\
\hline$C(22)-C(23)$ & $1.419(5)$ \\
\hline$C(22)-C(27)$ & $1.491(5)$ \\
\hline $\mathrm{C}(22)-\mathrm{Ga}(1)$ & $2.357(3)$ \\
\hline$C(23)-C(24)$ & $1.429(5)$ \\
\hline$C(23)-C(28)$ & $1.505(5)$ \\
\hline $\mathrm{C}(23)-\mathrm{Ga}(1)$ & $2.363(3)$ \\
\hline$C(24)-C(25)$ & $1.413(6)$ \\
\hline$C(24)-C(29)$ & $1.497(6)$ \\
\hline $\mathrm{C}(24)-\mathrm{Ga}(1)$ & $2.336(3)$ \\
\hline$C(25)-C(30)$ & $1.504(5)$ \\
\hline $\mathrm{C}(25)-\mathrm{Ga}(1)$ & $2.336(4)$ \\
\hline$C(31)-O(1)$ & $1.431(5)$ \\
\hline$C(31)-C(32)$ & $1.492(6)$ \\
\hline$C(32)-C(33)$ & $1.471(7)$ \\
\hline$C(33)-C(34)$ & $1.462(6)$ \\
\hline$C(34)-O(1)$ & $1.437(5)$ \\
\hline $\mathrm{O}(1)-\mathrm{Yb}(1)$ & $2.418(2)$ \\
\hline $\mathrm{Ga}(1)-\mathrm{Yb}(1)$ & $3.2872(4)$ \\
\hline$C(2)-C(1)-C(5)$ & $108.3(3)$ \\
\hline
\end{tabular}




\begin{tabular}{|c|c|}
\hline$C(2)-C(1)-C(6)$ & $125.3(4)$ \\
\hline$C(5)-C(1)-C(6)$ & $125.9(4)$ \\
\hline$C(2)-C(1)-Y b(1)$ & $74.5(2)$ \\
\hline$C(5)-C(1)-Y b(1)$ & $75.9(2)$ \\
\hline$C(6)-C(1)-Y b(1)$ & $122.0(2)$ \\
\hline$C(1)-C(2)-C(3)$ & $108.3(3)$ \\
\hline$C(1)-C(2)-C(7)$ & $125.4(4)$ \\
\hline$C(3)-C(2)-C(7)$ & $125.3(4)$ \\
\hline$C(1)-C(2)-Y b(1)$ & $75.4(2)$ \\
\hline$C(3)-C(2)-Y b(1)$ & $75.8(2)$ \\
\hline$C(7)-C(2)-Y b(1)$ & $124.6(3)$ \\
\hline$C(4)-C(3)-C(2)$ & $107.4(3)$ \\
\hline$C(4)-C(3)-C(8)$ & $125.3(4)$ \\
\hline$C(2)-C(3)-C(8)$ & $126.9(4)$ \\
\hline$C(4)-C(3)-Y b(1)$ & $75.9(2)$ \\
\hline$C(2)-C(3)-Y b(1)$ & $73.8(2)$ \\
\hline$C(8)-C(3)-Y b(1)$ & $121.5(3)$ \\
\hline$C(3)-C(4)-C(5)$ & $108.5(3)$ \\
\hline$C(3)-C(4)-C(9)$ & $126.2(4)$ \\
\hline$C(5)-C(4)-C(9)$ & $124.6(4)$ \\
\hline$C(3)-C(4)-Y b(1)$ & $74.3(2)$ \\
\hline$C(5)-C(4)-Y b(1)$ & $74.6(2)$ \\
\hline$C(9)-C(4)-Y b(1)$ & $124.8(3)$ \\
\hline$C(1)-C(5)-C(4)$ & $107.5(3)$ \\
\hline$C(1)-C(5)-C(10)$ & $126.5(3)$ \\
\hline$C(4)-C(5)-C(10)$ & $125.7(4)$ \\
\hline$C(1)-C(5)-Y b(1)$ & $74.0(2)$ \\
\hline$C(4)-C(5)-Y b(1)$ & $75.3(2)$ \\
\hline$C(10)-C(5)-Y b(1)$ & $122.0(2)$ \\
\hline$C(12)-C(11)-C(15)$ & $108.4(3)$ \\
\hline$C(12)-C(11)-C(16)$ & $126.0(4)$ \\
\hline$C(15)-C(11)-C(16)$ & $125.5(5)$ \\
\hline$C(12)-C(11)-Y b(1)$ & $75.8(2)$ \\
\hline$C(15)-C(11)-Y b(1)$ & $75.5(2)$ \\
\hline$C(16)-C(11)-Y b(1)$ & $118.2(3)$ \\
\hline$C(11)-C(12)-C(13)$ & $108.6(3)$ \\
\hline$C(11)-C(12)-C(17)$ & $126.0(4)$ \\
\hline$C(13)-C(12)-C(17)$ & $124.5(4)$ \\
\hline $\mathrm{C}(11)-\mathrm{C}(12)-\mathrm{Yb}(1)$ & $74.5(2)$ \\
\hline$C(13)-C(12)-Y b(1)$ & $76.0(2)$ \\
\hline$C(17)-C(12)-Y b(1)$ & $124.7(3)$ \\
\hline$C(12)-C(13)-C(14)$ & $108.0(3)$ \\
\hline$C(12)-C(13)-C(18)$ & $124.3(4)$ \\
\hline$C(14)-C(13)-C(18)$ & $127.3(4)$ \\
\hline$C(12)-C(13)-Y b(1)$ & $74.2(2)$ \\
\hline$C(14)-C(13)-Y b(1)$ & $74.8(2)$ \\
\hline$C(18)-C(13)-Y b(1)$ & $122.6(3)$ \\
\hline$C(13)-C(14)-C(15)$ & $108.0(3)$ \\
\hline$C(13)-C(14)-C(19)$ & $126.7(5)$ \\
\hline$C(15)-C(14)-C(19)$ & $125.0(4)$ \\
\hline $\mathrm{C}(13)-\mathrm{C}(14)-\mathrm{Yb}(1)$ & $75.4(2)$ \\
\hline$C(15)-C(14)-Y b(1)$ & $74.4(2)$ \\
\hline $\mathrm{C}(19)-\mathrm{C}(14)-\mathrm{Yb}(1)$ & $122.0(3)$ \\
\hline$C(14)-C(15)-C(11)$ & $107.1(3)$ \\
\hline$C(14)-C(15)-C(20)$ & $124.7(5)$ \\
\hline$C(11)-C(15)-C(20)$ & $127.5(5)$ \\
\hline$C(14)-C(15)-Y b(1)$ & $75.6(2)$ \\
\hline$C(11)-C(15)-Y b(1)$ & $74.2(2)$ \\
\hline
\end{tabular}




$$
\begin{aligned}
& \mathrm{C}(20)-\mathrm{C}(15)-\mathrm{Yb}(1) \quad 123.8(3) \\
& C(25)-C(21)-C(22) \quad 108.5(3) \\
& C(25)-C(21)-C(26) \quad 126.2(4) \\
& C(22)-C(21)-C(26) \quad 125.1(4) \\
& \mathrm{C}(25)-\mathrm{C}(21)-\mathrm{Ga}(1) \quad 72.7(2) \\
& \mathrm{C}(22)-\mathrm{C}(21)-\mathrm{Ga}(1) \quad 73.3(2) \\
& \mathrm{C}(26)-\mathrm{C}(21)-\mathrm{Ga}(1) \quad 124.2(3) \\
& \mathrm{C}(23)-\mathrm{C}(22)-\mathrm{C}(21) \quad 107.6(3) \\
& C(23)-C(22)-C(27) \quad 126.6(4) \\
& C(21)-C(22)-C(27) \quad 125.6(4) \\
& \mathrm{C}(23)-\mathrm{C}(22)-\mathrm{Ga}(1) \quad 72.7(2) \\
& \mathrm{C}(21)-\mathrm{C}(22)-\mathrm{Ga}(1) \quad 71.4(2) \\
& \mathrm{C}(27)-\mathrm{C}(22)-\mathrm{Ga}(1) \quad 125.7(3) \\
& \mathrm{C}(22)-\mathrm{C}(23)-\mathrm{C}(24) \quad 107.8(3) \\
& \mathrm{C}(22)-\mathrm{C}(23)-\mathrm{C}(28) \quad 125.8(4) \\
& \mathrm{C}(24)-\mathrm{C}(23)-\mathrm{C}(28) \quad 126.2(4) \\
& \mathrm{C}(22)-\mathrm{C}(23)-\mathrm{Ga}(1) \quad 72.31(19) \\
& \mathrm{C}(24)-\mathrm{C}(23)-\mathrm{Ga}(1) \quad 71.28(19) \\
& \mathrm{C}(28)-\mathrm{C}(23)-\mathrm{Ga}(1) \quad 125.6(2) \\
& \mathrm{C}(25)-\mathrm{C}(24)-\mathrm{C}(23) \quad 107.6(3) \\
& C(25)-C(24)-C(29) \quad 126.8(4) \\
& C(23)-C(24)-C(29) \quad 125.3(4) \\
& \mathrm{C}(25)-\mathrm{C}(24)-\mathrm{Ga}(1) \quad 72.4(2) \\
& \mathrm{C}(23)-\mathrm{C}(24)-\mathrm{Ga}(1) \quad 73.3(2) \\
& \mathrm{C}(29)-\mathrm{C}(24)-\mathrm{Ga}(1) \quad 124.7 \text { (3) } \\
& C(21)-C(25)-C(24) \quad 108.6(3) \\
& C(21)-C(25)-C(30) \quad 126.4(4) \\
& \mathrm{C}(24)-\mathrm{C}(25)-\mathrm{C}(30) \quad 124.9(4) \\
& \mathrm{C}(21)-\mathrm{C}(25)-\mathrm{Ga}(1) \quad 72.4(2) \\
& \mathrm{C}(24)-\mathrm{C}(25)-\mathrm{Ga}(1) \quad 72.4(2) \\
& \mathrm{C}(30)-\mathrm{C}(25)-\mathrm{Ga}(1) \quad 123.5(3) \\
& \mathrm{O}(1)-\mathrm{C}(31)-\mathrm{C}(32) \quad 105.6(4) \\
& \mathrm{C}(33)-\mathrm{C}(32)-\mathrm{C}(31) \quad 104.0(4) \\
& \mathrm{C}(34)-\mathrm{C}(33)-\mathrm{C}(32) \quad 107.1(4) \\
& \mathrm{O}(1)-\mathrm{C}(34)-\mathrm{C}(33) \quad 107.5(4) \\
& \mathrm{C}(31)-\mathrm{O}(1)-\mathrm{C}(34) \quad 107.3(3) \\
& \mathrm{C}(31)-\mathrm{O}(1)-\mathrm{Yb}(1) \quad 131.6(3) \\
& \mathrm{C}(34)-\mathrm{O}(1)-\mathrm{Yb}(1) \quad 121.0(2) \\
& \mathrm{C}(21)-\mathrm{Ga}(1)-\mathrm{C}(24) \quad 58.60(13) \\
& \mathrm{C}(21)-\mathrm{Ga}(1)-\mathrm{C}(25) \quad 34.92(14) \\
& \mathrm{C}(24)-\mathrm{Ga}(1)-\mathrm{C}(25) \quad 35.22(14) \\
& \mathrm{C}(21)-\mathrm{Ga}(1)-\mathrm{C}(22) \quad 35.29(13) \\
& \mathrm{C}(24)-\mathrm{Ga}(1)-\mathrm{C}(22) \quad 58.72(13) \\
& \mathrm{C}(25)-\mathrm{Ga}(1)-\mathrm{C}(22) \quad 58.41(13) \\
& \mathrm{C}(21)-\mathrm{Ga}(1)-\mathrm{C}(23) \quad 58.42(12) \\
& \mathrm{C}(24)-\mathrm{Ga}(1)-\mathrm{C}(23) \quad 35.42(13) \\
& \mathrm{C}(25)-\mathrm{Ga}(1)-\mathrm{C}(23) \quad 58.43(13) \\
& \mathrm{C}(22)-\mathrm{Ga}(1)-\mathrm{C}(23) \quad 34.98(12) \\
& \mathrm{C}(21)-\mathrm{Ga}(1)-\mathrm{Yb}(1) \quad 152.44(10) \\
& \mathrm{C}(24)-\mathrm{Ga}(1)-\mathrm{Yb}(1) \quad 147.05(9) \\
& \mathrm{C}(25)-\mathrm{Ga}(1)-\mathrm{Yb}(1) \quad 151.82(9) \\
& \mathrm{C}(22)-\mathrm{Ga}(1)-\mathrm{Yb}(1) \quad 148.21(9) \\
& \mathrm{C}(23)-\mathrm{Ga}(1)-\mathrm{Yb}(1) \quad 145.36(9) \\
& \mathrm{O}(1)-\mathrm{Yb}(1)-\mathrm{C}(2) \quad 131.55(10) \\
& \mathrm{O}(1)-\mathrm{Yb}(1)-\mathrm{C}(11) \quad 129.79(11) \\
& \mathrm{C}(2)-\mathrm{Yb}(1)-\mathrm{C}(11) \quad 96.82(12) \\
& \mathrm{O}(1)-\mathrm{Yb}(1)-\mathrm{C}(1) \quad 119.51(11) \\
& \mathrm{C}(2)-\mathrm{Yb}(1)-\mathrm{C}(1) \\
& 30.12(12)
\end{aligned}
$$




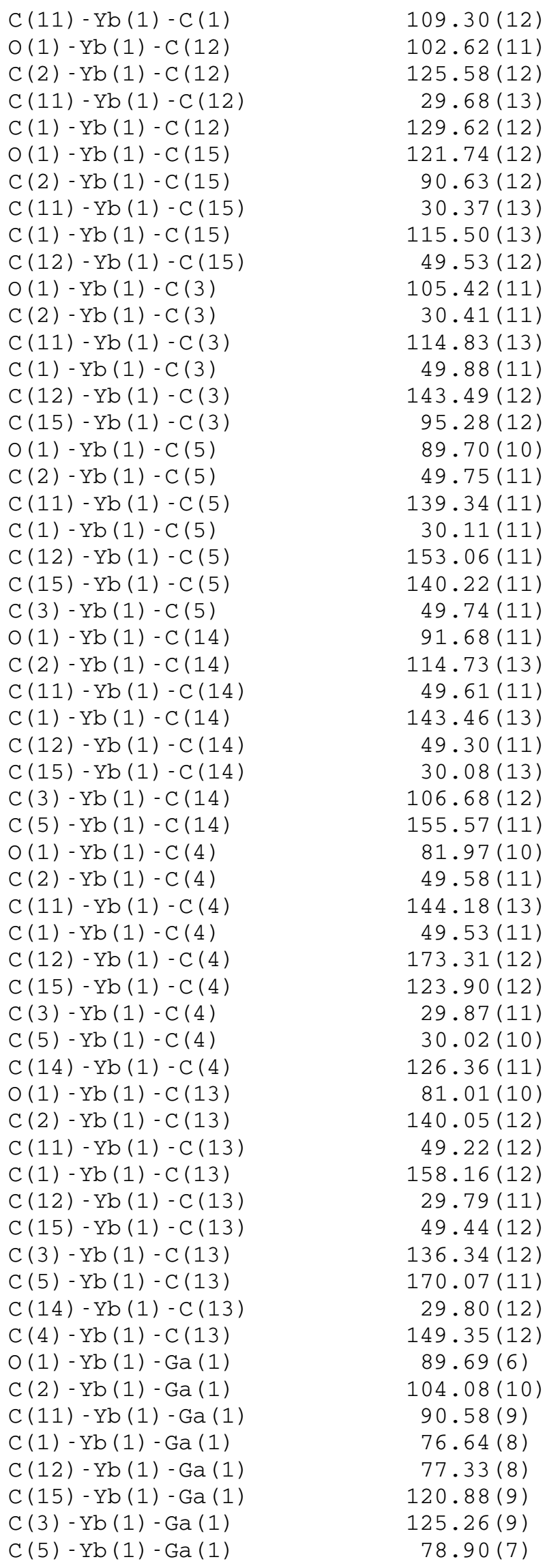



$\mathrm{C}(14)-\mathrm{Yb}(1)-\mathrm{Ga}(1)$
$125.49(8)$
$\mathrm{C}(4)-\mathrm{Yb}(1)-\mathrm{Ga}(1)$
$107.78(8)$
$\mathrm{C}(13)-\mathrm{Yb}(1)-\mathrm{Ga}(1)$
$97.44(9)$

Symmetry transformations used to generate equivalent atoms: 
Table 4. Anisotropic displacement parameters $(A \wedge 2 \times 10 \wedge 3)$ for 2 . The anisotropic displacement factor exponent takes the form: -2 pi^2 [ h^2 a*^2 U11 + .. + $2 h k a * b * U 12]$

\begin{tabular}{|c|c|c|c|c|c|c|}
\hline & U11 & $\mathrm{U} 22$ & U33 & U23 & U13 & $\mathrm{U} 12$ \\
\hline$C(1)$ & $49(3)$ & $38(2)$ & $23(2)$ & $-4(2)$ & $-4(2)$ & $14(2)$ \\
\hline$C(2)$ & $33(2)$ & $55(2)$ & $27(2)$ & $-6(2)$ & $-6(2)$ & $6(2)$ \\
\hline$C(3)$ & $41(2)$ & $39(2)$ & $32(2)$ & $-8(2)$ & $-2(2)$ & $-2(2)$ \\
\hline$C(4)$ & $39(2)$ & $39(2)$ & $30(2)$ & $-4(2)$ & $5(2)$ & $8(2)$ \\
\hline$C(5)$ & $33(2)$ & $35(2)$ & $26(2)$ & $-3(2)$ & $2(2)$ & $-1(2)$ \\
\hline$C(6)$ & $88(4)$ & $46(3)$ & $43(2)$ & $-2(2)$ & $-20(2)$ & $22(3)$ \\
\hline$C(7)$ & $39(2)$ & $101(4)$ & $44(3)$ & $-7(3)$ & $-10(2)$ & $12(3)$ \\
\hline$C(8)$ & $74(4)$ & $50(3)$ & $59(3)$ & $-19(2)$ & $4(3)$ & $-16(3)$ \\
\hline$C(9)$ & $55(3)$ & $63(3)$ & $53(3)$ & $-6(2)$ & $9(2)$ & $21(2)$ \\
\hline$C(10)$ & $53(3)$ & $57(3)$ & $44(2)$ & $3(2)$ & $10(2)$ & $-13(2)$ \\
\hline$C(11)$ & $43(2)$ & $48(3)$ & $37(2)$ & $10(2)$ & $14(2)$ & $19(2)$ \\
\hline$C(12)$ & $56(3)$ & $32(2)$ & $30(2)$ & $0(2)$ & $10(2)$ & $-6(2)$ \\
\hline$C(13)$ & $33(2)$ & $47(2)$ & $35(2)$ & $13(2)$ & $2(2)$ & $-4(2)$ \\
\hline$C(14)$ & $47(2)$ & $25(2)$ & $50(2)$ & $4(2)$ & $23(2)$ & $2(2)$ \\
\hline$C(15)$ & $33(2)$ & $68(3)$ & $32(2)$ & $-13(2)$ & $10(2)$ & $-16(2)$ \\
\hline$C(16)$ & $83(4)$ & $127(5)$ & $66(4)$ & $38(3)$ & $27(3)$ & $68(4)$ \\
\hline$C(17)$ & $139(6)$ & $51(3)$ & $57(3)$ & $-23(2)$ & $47(3)$ & $-36(3)$ \\
\hline $\mathrm{C}(18)$ & $55(3)$ & $128(5)$ & $51(3)$ & $29(3)$ & $-1(2)$ & $4(3)$ \\
\hline$C(19)$ & $100(4)$ & $34(2)$ & $118(5)$ & $4(3)$ & $68(4)$ & $1(3)$ \\
\hline$C(20)$ & $60(3)$ & $162(6)$ & $59(4)$ & $-28(4)$ & $12(3)$ & $-59(4)$ \\
\hline$C(21)$ & $33(2)$ & $32(2)$ & $47(2)$ & $5(2)$ & $-6(2)$ & $-6(2)$ \\
\hline$C(22)$ & $37(2)$ & $29(2)$ & $34(2)$ & $2(2)$ & $6(2)$ & $-3(2)$ \\
\hline$C(23)$ & $35(2)$ & $23(2)$ & $39(2)$ & $2(2)$ & $-5(2)$ & $-3(2)$ \\
\hline$C(24)$ & $41(2)$ & $24(2)$ & $45(2)$ & $-3(2)$ & $12(2)$ & $-4(2)$ \\
\hline$C(25)$ & $68(3)$ & $31(2)$ & $25(2)$ & $5(2)$ & $-3(2)$ & $-13(2)$ \\
\hline$C(26)$ & $43(3)$ & $78(3)$ & $103(4)$ & $18(3)$ & $-26(3)$ & $-1(3)$ \\
\hline$C(27)$ & $69(3)$ & $45(3)$ & $51(3)$ & $6(2)$ & $25(2)$ & $-6(2)$ \\
\hline$C(28)$ & $58(3)$ & $36(2)$ & $71(3)$ & $8(2)$ & $-27(2)$ & $2(2)$ \\
\hline C (29) & $75(3)$ & $43(3)$ & $92(4)$ & $-2(2)$ & $46(3)$ & $-1(3)$ \\
\hline$C(30)$ & $124(5)$ & $59(3)$ & $37(3)$ & $-3(2)$ & $-7(3)$ & $-27(3)$ \\
\hline$C(31)$ & $41(2)$ & $55(3)$ & $92(4)$ & $-2(3)$ & $-16(2)$ & $-8(2)$ \\
\hline$C(32)$ & $32(2)$ & $85(3)$ & $61(3)$ & $0(3)$ & $-4(2)$ & $-1(2)$ \\
\hline$C(33)$ & $41(3)$ & $65(3)$ & $136(6)$ & $13(3)$ & $4(3)$ & $15(3)$ \\
\hline$C(34)$ & $46(3)$ & $41(2)$ & $80(3)$ & $4(2)$ & $3(2)$ & $5(2)$ \\
\hline$O(1)$ & $29(1)$ & $49(2)$ & $48(2)$ & $1(1)$ & $-6(1)$ & $5(1)$ \\
\hline $\mathrm{Ga}(1)$ & $43(1)$ & $26(1)$ & $34(1)$ & $0(1)$ & $3(1)$ & $-3(1)$ \\
\hline $\mathrm{Yb}(1)$ & $25(1)$ & $28(1)$ & $26(1)$ & $-1(1)$ & $0(1)$ & $1(1)$ \\
\hline
\end{tabular}


Table 5. Hydrogen coordinates $(\mathrm{x} 10 \wedge 4)$ and isotropic displacement parameters $(A \wedge 2 \times 10 \wedge 3)$ for 2 .

\begin{tabular}{|c|c|c|c|c|}
\hline & $\mathrm{x}$ & $y$ & $z$ & $\mathrm{U}(\mathrm{eq})$ \\
\hline $\mathrm{H}(6 \mathrm{~A})$ & 2126 & 10219 & -184 & 89 \\
\hline $\mathrm{H}(6 \mathrm{~B})$ & 1172 & 10040 & -34 & 89 \\
\hline $\mathrm{H}(6 \mathrm{C})$ & 1528 & 10046 & -852 & 89 \\
\hline $\mathrm{H}(7 \mathrm{~A})$ & 210 & 9202 & -103 & 92 \\
\hline $\mathrm{H}(7 \mathrm{~B})$ & 188 & 8507 & -239 & 92 \\
\hline $\mathrm{H}(7 \mathrm{C})$ & 336 & 8946 & -911 & 92 \\
\hline $\mathrm{H}(8 \mathrm{~A})$ & 1546 & 7599 & -883 & 91 \\
\hline $\mathrm{H}(8 \mathrm{~B})$ & 978 & 7679 & -169 & 91 \\
\hline $\mathrm{H}(8 \mathrm{C})$ & 1914 & 7436 & -95 & 91 \\
\hline $\mathrm{H}(9 \mathrm{~A})$ & 3315 & 7696 & -246 & 85 \\
\hline $\mathrm{H}(9 \mathrm{~B})$ & 3936 & 8241 & -132 & 85 \\
\hline $\mathrm{H}(9 \mathrm{C})$ & 3592 & 8088 & -932 & 85 \\
\hline $\mathrm{H}(10 \mathrm{~A})$ & 3422 & 9884 & -194 & 77 \\
\hline $\mathrm{H}(10 \mathrm{~B})$ & 3724 & 9474 & -854 & 77 \\
\hline $\mathrm{H}(10 \mathrm{C})$ & 3976 & 9312 & -31 & 77 \\
\hline $\mathrm{H}(16 \mathrm{~A})$ & -279 & 9544 & 2005 & 138 \\
\hline $\mathrm{H}(16 \mathrm{~B})$ & -252 & 9273 & 1198 & 138 \\
\hline $\mathrm{H}(16 \mathrm{C})$ & 390 & 9781 & 1424 & 138 \\
\hline $\mathrm{H}(17 \mathrm{~A})$ & 1199 & 9583 & 3260 & 123 \\
\hline $\mathrm{H}(17 \mathrm{~B})$ & 1293 & 9955 & 2522 & 123 \\
\hline $\mathrm{H}(17 \mathrm{C})$ & 2099 & 9658 & 2887 & 123 \\
\hline $\mathrm{H}(18 \mathrm{~A})$ & 2909 & 8666 & 2929 & 117 \\
\hline $\mathrm{H}(18 \mathrm{~B})$ & 2580 & 8008 & 3050 & 117 \\
\hline $\mathrm{H}(18 \mathrm{C})$ & 2203 & 8535 & 3528 & 117 \\
\hline $\mathrm{H}(19 \mathrm{~A})$ & 1025 & 7240 & 2288 & 126 \\
\hline $\mathrm{H}(19 \mathrm{~B})$ & 2011 & 7358 & 2347 & 126 \\
\hline $\mathrm{H}(19 \mathrm{C})$ & 1578 & 7295 & 1561 & 126 \\
\hline $\mathrm{H}(20 \mathrm{~A})$ & 293 & 7825 & 838 & 141 \\
\hline $\mathrm{H}(20 \mathrm{~B})$ & -359 & 8340 & 1016 & 141 \\
\hline $\mathrm{H}(20 \mathrm{C})$ & -280 & 7788 & 1555 & 141 \\
\hline$H(26 A)$ & 4805 & 10529 & 1382 & 112 \\
\hline $\mathrm{H}(26 \mathrm{~B})$ & 4630 & 10576 & 2242 & 112 \\
\hline $\mathrm{H}(26 \mathrm{C})$ & 4889 & 11149 & 1789 & 112 \\
\hline $\mathrm{H}(27 \mathrm{~A})$ & 4092 & 11477 & 209 & 83 \\
\hline $\mathrm{H}(27 \mathrm{~B})$ & 3436 & 11052 & -189 & 83 \\
\hline $\mathrm{H}(27 \mathrm{C})$ & 4240 & 10781 & 210 & 83 \\
\hline $\mathrm{H}(28 \mathrm{~A})$ & 1338 & 11194 & 494 & 83 \\
\hline $\mathrm{H}(28 \mathrm{~B})$ & 2113 & 11300 & -44 & 83 \\
\hline $\mathrm{H}(28 \mathrm{C})$ & 1807 & 11816 & 487 & 83 \\
\hline $\mathrm{H}(29 \mathrm{~A})$ & 1433 & 11068 & 2616 & 105 \\
\hline $\mathrm{H}(29 \mathrm{~B})$ & 1005 & 11102 & 1826 & 105 \\
\hline $\mathrm{H}(29 \mathrm{C})$ & 1404 & 11679 & 2184 & 105 \\
\hline $\mathrm{H}(30 \mathrm{~A})$ & 3393 & 11237 & 3161 & 110 \\
\hline $\mathrm{H}(30 \mathrm{~B})$ & 3477 & 10541 & 3062 & 110 \\
\hline $\mathrm{H}(30 \mathrm{C})$ & 2575 & 10831 & 3179 & 110 \\
\hline $\mathrm{H}(31 \mathrm{~A})$ & 4288 & 8935 & 2073 & 75 \\
\hline $\mathrm{H}(31 \mathrm{~B})$ & 4280 & 9147 & 1233 & 75 \\
\hline $\mathrm{H}(32 \mathrm{~A})$ & 5094 & 8400 & 866 & 71 \\
\hline $\mathrm{H}(32 \mathrm{~B})$ & 5429 & 8450 & 1695 & 71 \\
\hline
\end{tabular}




\begin{tabular}{lllll}
$\mathrm{H}(33 \mathrm{~A})$ & 4724 & 7507 & 1178 & 97 \\
$\mathrm{H}(33 \mathrm{~B})$ & 4760 & 7657 & 2037 & 97 \\
$\mathrm{H}(34 \mathrm{~A})$ & 3392 & 7633 & 1173 & 67 \\
$\mathrm{H}(34 \mathrm{~B})$ & 3422 & 7748 & 2040 & 67 \\
\hline
\end{tabular}


Table 6. Torsion angles [deg] for 2 .

\begin{tabular}{|c|c|}
\hline$C(5)-C(1)-C(2)-C(3)$ & $-0.3(4)$ \\
\hline$C(6)-C(1)-C(2)-C(3)$ & $172.2(4)$ \\
\hline $\mathrm{Yb}(1)-\mathrm{C}(1)-\mathrm{C}(2)-\mathrm{C}(3)$ & $-69.3(3)$ \\
\hline$C(5)-C(1)-C(2)-C(7)$ & $-168.7(4)$ \\
\hline$C(6)-C(1)-C(2)-C(7)$ & $3.7(6)$ \\
\hline $\mathrm{Yb}(1)-\mathrm{C}(1)-\mathrm{C}(2)-\mathrm{C}(7)$ & $122.3(4)$ \\
\hline$C(5)-C(1)-C(2)-Y b(1)$ & $69.0(2)$ \\
\hline$C(6)-C(1)-C(2)-Y b(1)$ & $-118.6(4)$ \\
\hline$C(1)-C(2)-C(3)-C(4)$ & $0.0(4)$ \\
\hline$C(7)-C(2)-C(3)-C(4)$ & $168.4(4)$ \\
\hline $\mathrm{Yb}(1)-\mathrm{C}(2)-\mathrm{C}(3)-\mathrm{C}(4)$ & $-69.1(3)$ \\
\hline$C(1)-C(2)-C(3)-C(8)$ & $-173.3(4)$ \\
\hline$C(7)-C(2)-C(3)-C(8)$ & $-4.9(6)$ \\
\hline $\mathrm{Yb}(1)-\mathrm{C}(2)-\mathrm{C}(3)-\mathrm{C}(8)$ & $117.6(4)$ \\
\hline$C(1)-C(2)-C(3)-Y b(1)$ & $69.0(2)$ \\
\hline$C(7)-C(2)-C(3)-Y b(1)$ & $-122.5(4)$ \\
\hline$C(2)-C(3)-C(4)-C(5)$ & $0.3(4)$ \\
\hline$C(8)-C(3)-C(4)-C(5)$ & $173.7(4)$ \\
\hline $\mathrm{Yb}(1)-\mathrm{C}(3)-\mathrm{C}(4)-\mathrm{C}(5)$ & $-67.4(3)$ \\
\hline$C(2)-C(3)-C(4)-C(9)$ & $-170.5(4)$ \\
\hline$C(8)-C(3)-C(4)-C(9)$ & $3.0(6)$ \\
\hline $\mathrm{Yb}(1)-\mathrm{C}(3)-\mathrm{C}(4)-\mathrm{C}(9)$ & $121.9(4)$ \\
\hline$C(2)-C(3)-C(4)-Y b(1)$ & $67.7(3)$ \\
\hline$C(8)-C(3)-C(4)-Y b(1)$ & $-118.9(4)$ \\
\hline$C(2)-C(1)-C(5)-C(4)$ & $0.4(4)$ \\
\hline$C(6)-C(1)-C(5)-C(4)$ & $-171.9(4)$ \\
\hline $\mathrm{Yb}(1)-\mathrm{C}(1)-\mathrm{C}(5)-\mathrm{C}(4)$ & $68.5(3)$ \\
\hline$C(2)-C(1)-C(5)-C(10)$ & $173.6(4)$ \\
\hline$C(6)-C(1)-C(5)-C(10)$ & $1.3(6)$ \\
\hline $\mathrm{Yb}(1)-\mathrm{C}(1)-\mathrm{C}(5)-\mathrm{C}(10)$ & $-118.3(4)$ \\
\hline$C(2)-C(1)-C(5)-Y b(1)$ & $-68.1(3)$ \\
\hline$C(6)-C(1)-C(5)-Y b(1)$ & $119.6(4)$ \\
\hline$C(3)-C(4)-C(5)-C(1)$ & $-0.4(4)$ \\
\hline$C(9)-C(4)-C(5)-C(1)$ & $170.5(4)$ \\
\hline $\mathrm{Yb}(1)-\mathrm{C}(4)-\mathrm{C}(5)-\mathrm{C}(1)$ & $-67.6(2)$ \\
\hline$C(3)-C(4)-C(5)-C(10)$ & $-173.7(4)$ \\
\hline$C(9)-C(4)-C(5)-C(10)$ & $-2.8(6)$ \\
\hline $\mathrm{Yb}(1)-\mathrm{C}(4)-\mathrm{C}(5)-\mathrm{C}(10)$ & $119.1(4)$ \\
\hline$C(3)-C(4)-C(5)-Y b(1)$ & $67.1(3)$ \\
\hline$C(9)-C(4)-C(5)-Y b(1)$ & $-121.9(4)$ \\
\hline$C(15)-C(11)-C(12)-C(13)$ & $0.0(4)$ \\
\hline$C(16)-C(11)-C(12)-C(13)$ & $176.2(4)$ \\
\hline $\mathrm{Yb}(1)-\mathrm{C}(11)-\mathrm{C}(12)-\mathrm{C}(13)$ & $-69.1(3)$ \\
\hline$C(15)-C(11)-C(12)-C(17)$ & $-169.0(4)$ \\
\hline$C(16)-C(11)-C(12)-C(17)$ & $7.2(7)$ \\
\hline $\mathrm{Yb}(1)-\mathrm{C}(11)-\mathrm{C}(12)-\mathrm{C}(17)$ & $121.9(4)$ \\
\hline$C(15)-C(11)-C(12)-Y b(1)$ & $69.1(3)$ \\
\hline$C(16)-C(11)-C(12)-Y b(1)$ & $-114.7(4)$ \\
\hline$C(11)-C(12)-C(13)-C(14)$ & $0.3(4)$ \\
\hline$C(17)-C(12)-C(13)-C(14)$ & $169.5(4)$ \\
\hline $\mathrm{Yb}(1)-\mathrm{C}(12)-\mathrm{C}(13)-\mathrm{C}(14)$ & $-67.8(3)$ \\
\hline$C(11)-C(12)-C(13)-C(18)$ & $-173.0(4)$ \\
\hline$C(17)-C(12)-C(13)-C(18)$ & $-3.8(6)$ \\
\hline $\mathrm{Yb}(1)-\mathrm{C}(12)-\mathrm{C}(13)-\mathrm{C}(18)$ & $118.9(4)$ \\
\hline
\end{tabular}




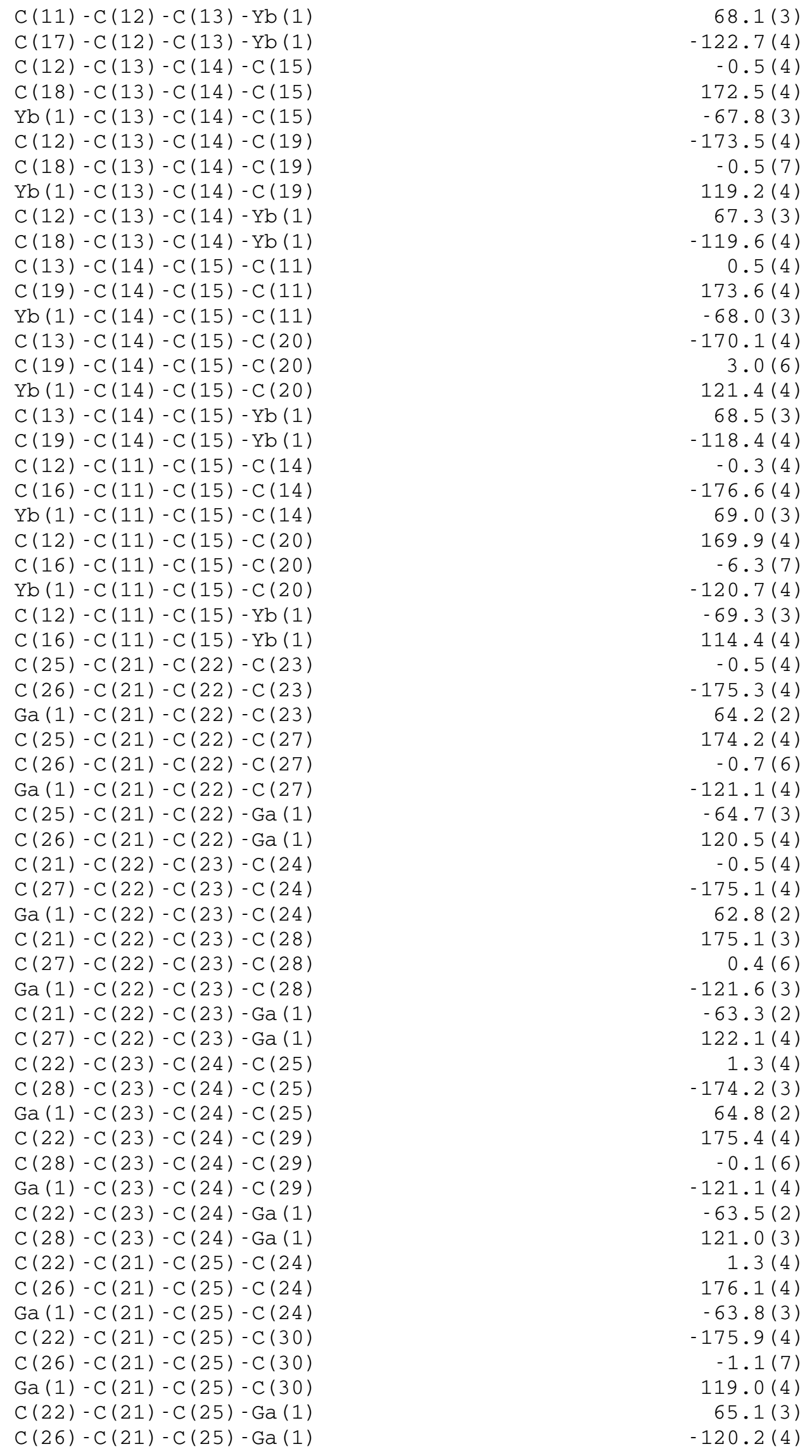




\begin{tabular}{|c|c|}
\hline$C(23)-C(24)-C(25)-C(21)$ & $-1.6(4)$ \\
\hline$C(29)-C(24)-C(25)-C(21)$ & $-175.6(4)$ \\
\hline $\mathrm{Ga}(1)-\mathrm{C}(24)-\mathrm{C}(25)-\mathrm{C}(21)$ & $63.8(3)$ \\
\hline$C(23)-C(24)-C(25)-C(30)$ & $175.6(4)$ \\
\hline$C(29)-C(24)-C(25)-C(30)$ & $1.6(6)$ \\
\hline $\mathrm{Ga}(1)-\mathrm{C}(24)-\mathrm{C}(25)-\mathrm{C}(30)$ & $-119.0(4)$ \\
\hline$C(23)-C(24)-C(25)-G a(1)$ & $-65.4(2)$ \\
\hline$C(29)-C(24)-C(25)-G a(1)$ & $120.6(4)$ \\
\hline $\mathrm{O}(1)-\mathrm{C}(31)-\mathrm{C}(32)-\mathrm{C}(33)$ & $-28.5(5)$ \\
\hline$C(31)-C(32)-C(33)-C(34)$ & $19.1(6)$ \\
\hline$C(32)-C(33)-C(34)-O(1)$ & $-2.9(6)$ \\
\hline $\mathrm{C}(32)-\mathrm{C}(31)-\mathrm{O}(1)-\mathrm{C}(34)$ & $27.5(5)$ \\
\hline$C(32)-C(31)-O(1)-Y b(1)$ & $-149.9(3)$ \\
\hline $\mathrm{C}(33)-\mathrm{C}(34)-\mathrm{O}(1)-\mathrm{C}(31)$ & $-15.5(5)$ \\
\hline$C(33)-C(34)-O(1)-Y b(1)$ & $162.2(4)$ \\
\hline $\mathrm{C}(25)-\mathrm{C}(21)-\mathrm{Ga}(1)-\mathrm{C}(24)$ & $37.1(2)$ \\
\hline $\mathrm{C}(22)-\mathrm{C}(21)-\mathrm{Ga}(1)-\mathrm{C}(24)$ & $-79.0(2)$ \\
\hline $\mathrm{C}(26)-\mathrm{C}(21)-\mathrm{Ga}(1)-\mathrm{C}(24)$ & $159.5(4)$ \\
\hline$C(22)-C(21)-G a(1)-C(25)$ & $-116.1(3)$ \\
\hline $\mathrm{C}(26)-\mathrm{C}(21)-\mathrm{Ga}(1)-\mathrm{C}(25)$ & $122.4(5)$ \\
\hline $\mathrm{C}(25)-\mathrm{C}(21)-\mathrm{Ga}(1)-\mathrm{C}(22)$ & $116.1(3)$ \\
\hline $\mathrm{C}(26)-\mathrm{C}(21)-\mathrm{Ga}(1)-\mathrm{C}(22)$ & $-121.5(5)$ \\
\hline$C(25)-C(21)-G a(1)-C(23)$ & $78.9(2)$ \\
\hline $\mathrm{C}(22)-\mathrm{C}(21)-\mathrm{Ga}(1)-\mathrm{C}(23)$ & $-37.2(2)$ \\
\hline$C(26)-C(21)-G a(1)-C(23)$ & $-158.7(4)$ \\
\hline $\mathrm{C}(25)-\mathrm{C}(21)-\mathrm{Ga}(1)-\mathrm{Yb}(1)$ & $-125.7(2)$ \\
\hline $\mathrm{C}(22)-\mathrm{C}(21)-\mathrm{Ga}(1)-\mathrm{Yb}(1)$ & $118.2(2)$ \\
\hline $\mathrm{C}(26)-\mathrm{C}(21)-\mathrm{Ga}(1)-\mathrm{Yb}(1)$ & $-3.3(5)$ \\
\hline $\mathrm{C}(25)-\mathrm{C}(24)-\mathrm{Ga}(1)-\mathrm{C}(21)$ & $-36.8(2)$ \\
\hline $\mathrm{C}(23)-\mathrm{C}(24)-\mathrm{Ga}(1)-\mathrm{C}(21)$ & $78.4(2)$ \\
\hline $\mathrm{C}(29)-\mathrm{C}(24)-\mathrm{Ga}(1)-\mathrm{C}(21)$ & $-159.8(4)$ \\
\hline $\mathrm{C}(23)-\mathrm{C}(24)-\mathrm{Ga}(1)-\mathrm{C}(25)$ & $115.2(3)$ \\
\hline $\mathrm{C}(29)-\mathrm{C}(24)-\mathrm{Ga}(1)-\mathrm{C}(25)$ & $-123.0(5)$ \\
\hline $\mathrm{C}(25)-\mathrm{C}(24)-\mathrm{Ga}(1)-\mathrm{C}(22)$ & $-78.3(2)$ \\
\hline $\mathrm{C}(23)-\mathrm{C}(24)-\mathrm{Ga}(1)-\mathrm{C}(22)$ & $36.9(2)$ \\
\hline $\mathrm{C}(29)-\mathrm{C}(24)-\mathrm{Ga}(1)-\mathrm{C}(22)$ & $158.6(4)$ \\
\hline $\mathrm{C}(25)-\mathrm{C}(24)-\mathrm{Ga}(1)-\mathrm{C}(23)$ & $-115.2(3)$ \\
\hline $\mathrm{C}(29)-\mathrm{C}(24)-\mathrm{Ga}(1)-\mathrm{C}(23)$ & $121.7(5)$ \\
\hline $\mathrm{C}(25)-\mathrm{C}(24)-\mathrm{Ga}(1)-\mathrm{Yb}(1)$ & $128.7(2)$ \\
\hline $\mathrm{C}(23)-\mathrm{C}(24)-\mathrm{Ga}(1)-\mathrm{Yb}(1)$ & $-116.1(2)$ \\
\hline $\mathrm{C}(29)-\mathrm{C}(24)-\mathrm{Ga}(1)-\mathrm{Yb}(1)$ & $5.6(5)$ \\
\hline $\mathrm{C}(24)-\mathrm{C}(25)-\mathrm{Ga}(1)-\mathrm{C}(21)$ & $116.8(3)$ \\
\hline $\mathrm{C}(30)-\mathrm{C}(25)-\mathrm{Ga}(1)-\mathrm{C}(21)$ & $-122.5(5)$ \\
\hline $\mathrm{C}(21)-\mathrm{C}(25)-\mathrm{Ga}(1)-\mathrm{C}(24)$ & $-116.8(3)$ \\
\hline $\mathrm{C}(30)-\mathrm{C}(25)-\mathrm{Ga}(1)-\mathrm{C}(24)$ & $120.7(5)$ \\
\hline $\mathrm{C}(21)-\mathrm{C}(25)-\mathrm{Ga}(1)-\mathrm{C}(22)$ & $-37.5(2)$ \\
\hline $\mathrm{C}(24)-\mathrm{C}(25)-\mathrm{Ga}(1)-\mathrm{C}(22)$ & $79.3(2)$ \\
\hline$C(30)-C(25)-G a(1)-C(22)$ & $-160.0(5)$ \\
\hline $\mathrm{C}(21)-\mathrm{C}(25)-\mathrm{Ga}(1)-\mathrm{C}(23)$ & $-78.8(2)$ \\
\hline $\mathrm{C}(24)-\mathrm{C}(25)-\mathrm{Ga}(1)-\mathrm{C}(23)$ & $38.0(2)$ \\
\hline$C(30)-C(25)-G a(1)-C(23)$ & $158.7(5)$ \\
\hline $\mathrm{C}(21)-\mathrm{C}(25)-\mathrm{Ga}(1)-\mathrm{Yb}(1)$ & $127.3(2)$ \\
\hline $\mathrm{C}(24)-\mathrm{C}(25)-\mathrm{Ga}(1)-\mathrm{Yb}(1)$ & $-115.9(2)$ \\
\hline $\mathrm{C}(30)-\mathrm{C}(25)-\mathrm{Ga}(1)-\mathrm{Yb}(1)$ & $4.8(6)$ \\
\hline $\mathrm{C}(23)-\mathrm{C}(22)-\mathrm{Ga}(1)-\mathrm{C}(21)$ & $-116.0(3)$ \\
\hline $\mathrm{C}(27)-\mathrm{C}(22)-\mathrm{Ga}(1)-\mathrm{C}(21)$ & $121.0(4)$ \\
\hline $\mathrm{C}(23)-\mathrm{C}(22)-\mathrm{Ga}(1)-\mathrm{C}(24)$ & $-37.3(2)$ \\
\hline $\mathrm{C}(21)-\mathrm{C}(22)-\mathrm{Ga}(1)-\mathrm{C}(24)$ & $78.6(2)$ \\
\hline
\end{tabular}




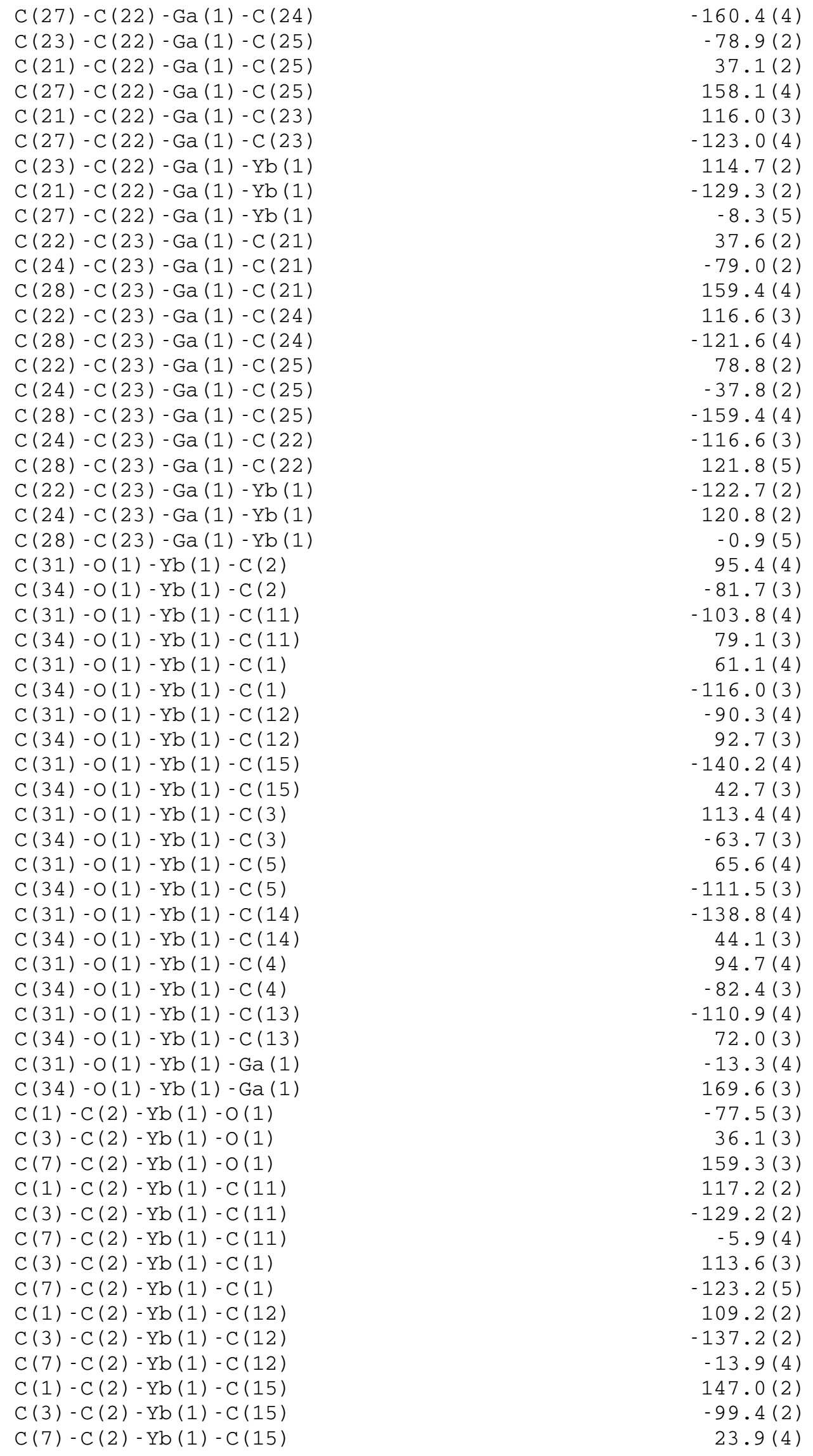




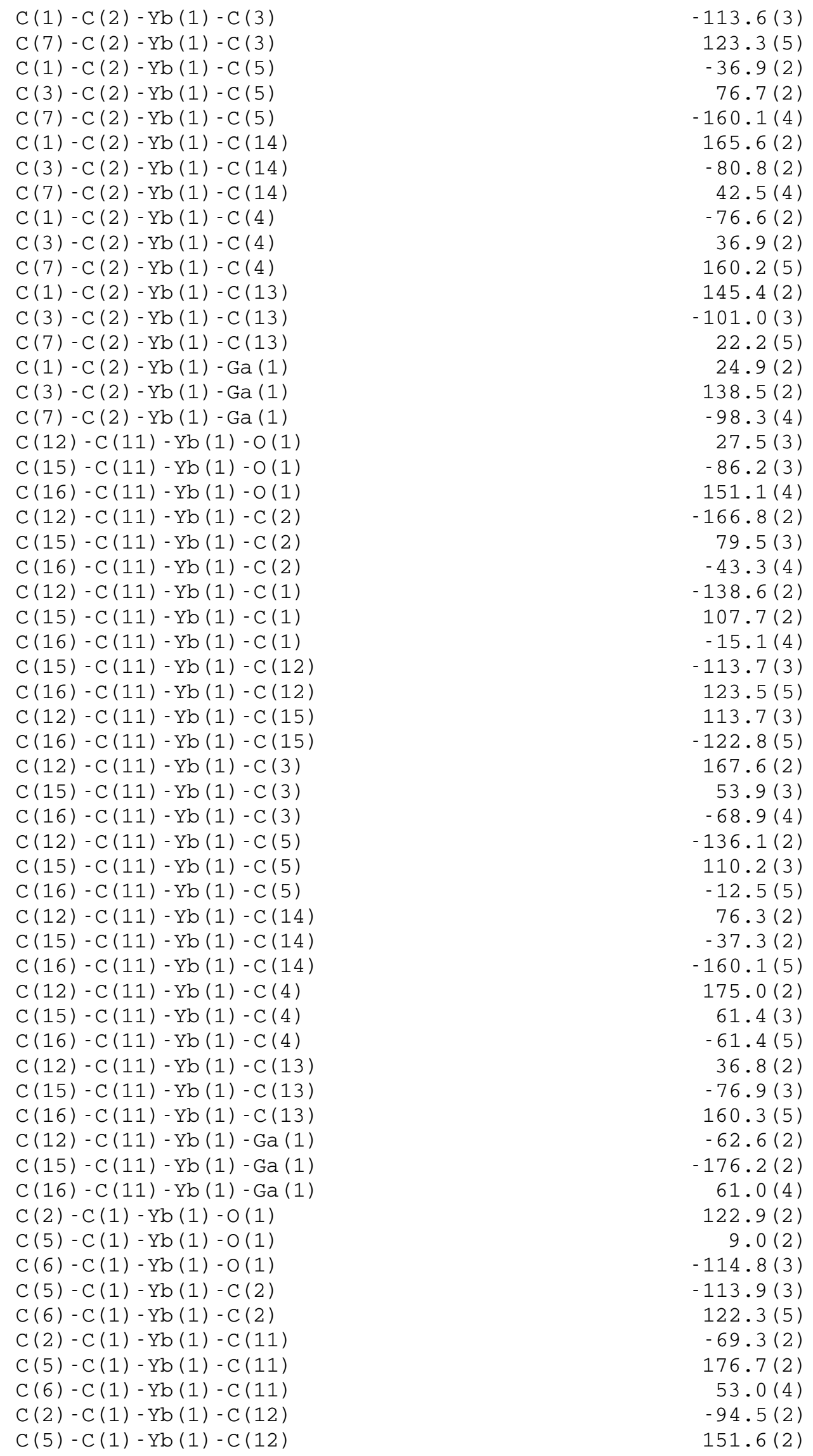




\begin{tabular}{|c|c|}
\hline$C(6)-C(1)-Y b(1)-C(12)$ & $27.8(4)$ \\
\hline$C(2)-C(1)-Y b(1)-C(15)$ & $-37.1(3)$ \\
\hline$C(5)-C(1)-Y b(1)-C(15)$ & $-151.0(2)$ \\
\hline$C(6)-C(1)-Y b(1)-C(15)$ & $85.2(4)$ \\
\hline$C(2)-C(1)-Y b(1)-C(3)$ & $37.3(2)$ \\
\hline$C(5)-C(1)-Y b(1)-C(3)$ & $-76.6(2)$ \\
\hline$C(6)-C(1)-Y b(1)-C(3)$ & $159.6(4)$ \\
\hline$C(2)-C(1)-Y b(1)-C(5)$ & $113.9(3)$ \\
\hline$C(6)-C(1)-Y b(1)-C(5)$ & $-123.8(4)$ \\
\hline$C(2)-C(1)-Y b(1)-C(14)$ & $-22.2(3)$ \\
\hline$C(5)-C(1)-Y b(1)-C(14)$ & $-136.2(2)$ \\
\hline$C(6)-C(1)-Y b(1)-C(14)$ & $100.1(4)$ \\
\hline$C(2)-C(1)-Y b(1)-C(4)$ & $76.8(2)$ \\
\hline$C(5)-C(1)-Y b(1)-C(4)$ & $-37.1(2)$ \\
\hline$C(6)-C(1)-Y b(1)-C(4)$ & $-160.9(4)$ \\
\hline$C(2)-C(1)-Y b(1)-C(13)$ & $-78.8(4)$ \\
\hline$C(5)-C(1)-Y b(1)-C(13)$ & $167.3(3)$ \\
\hline$C(6)-C(1)-Y b(1)-C(13)$ & $43.5(6)$ \\
\hline $\mathrm{C}(2)-\mathrm{C}(1)-\mathrm{Yb}(1)-\mathrm{Ga}(1)$ & $-155.2(2)$ \\
\hline $\mathrm{C}(5)-\mathrm{C}(1)-\mathrm{Yb}(1)-\mathrm{Ga}(1)$ & $90.9(2)$ \\
\hline$C(6)-C(1)-Y b(1)-G a(1)$ & $-32.9(3)$ \\
\hline$C(11)-C(12)-Y b(1)-O(1)$ & $-158.7(2)$ \\
\hline$C(13)-C(12)-Y b(1)-O(1)$ & $-44.5(2)$ \\
\hline $\mathrm{C}(17)-\mathrm{C}(12)-\mathrm{Yb}(1)-\mathrm{O}(1)$ & $78.0(4)$ \\
\hline$C(11)-C(12)-Y b(1)-C(2)$ & $16.2(3)$ \\
\hline $\mathrm{C}(13)-\mathrm{C}(12)-\mathrm{Yb}(1)-\mathrm{C}(2)$ & $130.4(2)$ \\
\hline$C(17)-C(12)-Y b(1)-C(2)$ & $-107.2(4)$ \\
\hline$C(13)-C(12)-Y b(1)-C(11)$ & $114.2(3)$ \\
\hline$C(17)-C(12)-Y b(1)-C(11)$ & $-123.3(5)$ \\
\hline $\mathrm{C}(11)-\mathrm{C}(12)-\mathrm{Yb}(1)-\mathrm{C}(1)$ & $54.1(3)$ \\
\hline$C(13)-C(12)-Y b(1)-C(1)$ & $168.3(2)$ \\
\hline$C(17)-C(12)-Y b(1)-C(1)$ & $-69.2(5)$ \\
\hline$C(11)-C(12)-Y b(1)-C(15)$ & $-37.5(2)$ \\
\hline$C(13)-C(12)-Y b(1)-C(15)$ & $76.7(2)$ \\
\hline$C(17)-C(12)-Y b(1)-C(15)$ & $-160.8(5)$ \\
\hline$C(11)-C(12)-Y b(1)-C(3)$ & $-19.2(3)$ \\
\hline$C(13)-C(12)-Y b(1)-C(3)$ & $95.0(3)$ \\
\hline$C(17)-C(12)-Y b(1)-C(3)$ & $-142.5(4)$ \\
\hline$C(11)-C(12)-Y b(1)-C(5)$ & $85.9(3)$ \\
\hline$C(13)-C(12)-Y b(1)-C(5)$ & $-159.9(2)$ \\
\hline$C(17)-C(12)-Y b(1)-C(5)$ & $-37.4(6)$ \\
\hline$C(11)-C(12)-Y b(1)-C(14)$ & $-77.5(3)$ \\
\hline$C(13)-C(12)-Y b(1)-C(14)$ & $36.7(2)$ \\
\hline$C(17)-C(12)-Y b(1)-C(14)$ & $159.2(5)$ \\
\hline$C(11)-C(12)-Y b(1)-C(4)$ & $-25.8(11)$ \\
\hline$C(13)-C(12)-Y b(1)-C(4)$ & $88.4(10)$ \\
\hline$C(17)-C(12)-Y b(1)-C(4)$ & $-149.1(9)$ \\
\hline$C(11)-C(12)-Y b(1)-C(13)$ & $-114.2(3)$ \\
\hline$C(17)-C(12)-Y b(1)-C(13)$ & $122.5(5)$ \\
\hline $\mathrm{C}(11)-\mathrm{C}(12)-\mathrm{Yb}(1)-\mathrm{Ga}(1)$ & $114.5(2)$ \\
\hline $\mathrm{C}(13)-\mathrm{C}(12)-\mathrm{Yb}(1)-\mathrm{Ga}(1)$ & $-131 \cdot 3(2)$ \\
\hline $\mathrm{C}(17)-\mathrm{C}(12)-\mathrm{Yb}(1)-\mathrm{Ga}(1)$ & $-8.8(4)$ \\
\hline $\mathrm{C}(14)-\mathrm{C}(15)-\mathrm{Yb}(1)-\mathrm{O}(1)$ & $2.8(3)$ \\
\hline$C(11)-C(15)-Y b(1)-O(1)$ & $115.6(2)$ \\
\hline$C(20)-C(15)-Y b(1)-O(1)$ & $-119.6(4)$ \\
\hline$C(14)-C(15)-Y b(1)-C(2)$ & $144.7(2)$ \\
\hline$C(11)-C(15)-Y b(1)-C(2)$ & $-102.5(3)$ \\
\hline$C(20)-C(15)-Y b(1)-C(2)$ & $22.3(5)$ \\
\hline
\end{tabular}




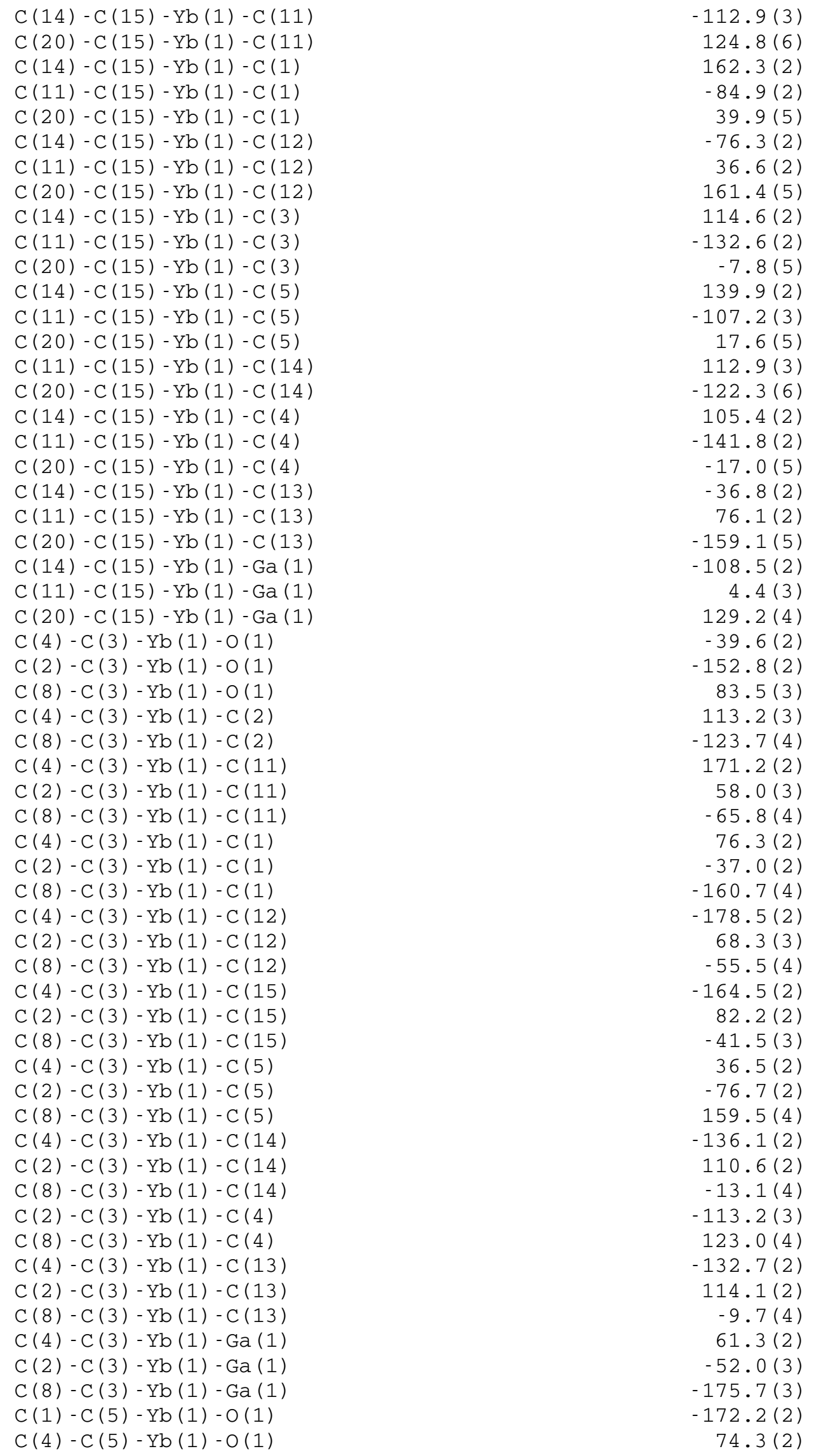




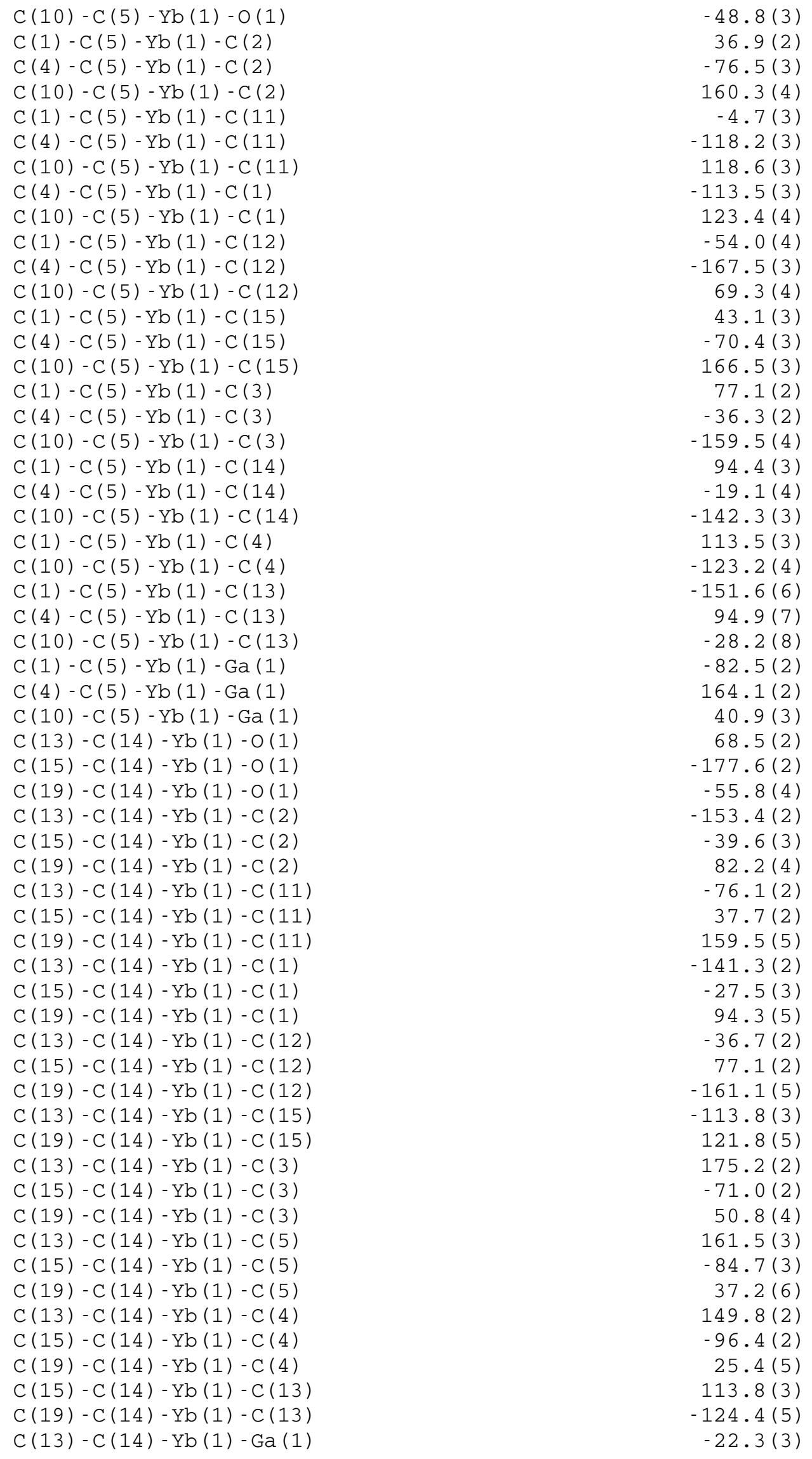




\begin{tabular}{|c|c|}
\hline $\mathrm{C}(15)-\mathrm{C}(14)-\mathrm{Yb}(1)-\mathrm{Ga}(1)$ & $91.5(2)$ \\
\hline $\mathrm{C}(19)-\mathrm{C}(14)-\mathrm{Yb}(1)-\mathrm{Ga}(1)$ & $-146.7(4)$ \\
\hline$C(3)-C(4)-Y b(1)-O(1)$ & $141.7(2)$ \\
\hline$C(5)-C(4)-Y b(1)-O(1)$ & $-103.5(2)$ \\
\hline$C(9)-C(4)-Y b(1)-O(1)$ & $18.2(3)$ \\
\hline$C(3)-C(4)-Y b(1)-C(2)$ & $-37.6(2)$ \\
\hline$C(5)-C(4)-Y b(1)-C(2)$ & $77.1(3)$ \\
\hline$C(9)-C(4)-Y b(1)-C(2)$ & $-161.1(4)$ \\
\hline$C(3)-C(4)-Y b(1)-C(11)$ & $-13.7(3)$ \\
\hline$C(5)-C(4)-Y b(1)-C(11)$ & $101.1(3)$ \\
\hline$C(9)-C(4)-Y b(1)-C(11)$ & $-137.1(3)$ \\
\hline$C(3)-C(4)-Y b(1)-C(1)$ & $-77.6(2)$ \\
\hline$C(5)-C(4)-Y b(1)-C(1)$ & $37.2(2)$ \\
\hline$C(9)-C(4)-Y b(1)-C(1)$ & $159.0(4)$ \\
\hline$C(3)-C(4)-Y b(1)-C(12)$ & $7.9(11)$ \\
\hline$C(5)-C(4)-Y b(1)-C(12)$ & $122.7(10)$ \\
\hline$C(9)-C(4)-Y b(1)-C(12)$ & $-115.5(10)$ \\
\hline$C(3)-C(4)-Y b(1)-C(15)$ & $18.6(3)$ \\
\hline$C(5)-C(4)-Y b(1)-C(15)$ & $133.4(2)$ \\
\hline$C(9)-C(4)-Y b(1)-C(15)$ & $-104.8(4)$ \\
\hline$C(5)-C(4)-Y b(1)-C(3)$ & $114.8(3)$ \\
\hline$C(9)-C(4)-Y b(1)-C(3)$ & $-123.5(4)$ \\
\hline$C(3)-C(4)-Y b(1)-C(5)$ & $-114.8(3)$ \\
\hline$C(9)-C(4)-Y b(1)-C(5)$ & $121.7(5)$ \\
\hline$C(3)-C(4)-Y b(1)-C(14)$ & $55.5(3)$ \\
\hline$C(5)-C(4)-Y b(1)-C(14)$ & $170.3(2)$ \\
\hline$C(9)-C(4)-Y b(1)-C(14)$ & $-67.9(4)$ \\
\hline$C(3)-C(4)-Y b(1)-C(13)$ & $84.9(3)$ \\
\hline$C(5)-C(4)-Y b(1)-C(13)$ & $-160.3(2)$ \\
\hline$C(9)-C(4)-Y b(1)-C(13)$ & $-38.6(5)$ \\
\hline $\mathrm{C}(3)-\mathrm{C}(4)-\mathrm{Yb}(1)-\mathrm{Ga}(1)$ & $-131.2(2)$ \\
\hline$C(5)-C(4)-Y b(1)-G a(1)$ & $-16.4(2)$ \\
\hline$C(9)-C(4)-Y b(1)-G a(1)$ & $105.3(3)$ \\
\hline $\mathrm{C}(12)-\mathrm{C}(13)-\mathrm{Yb}(1)-\mathrm{O}(1)$ & $136.2(2)$ \\
\hline $\mathrm{C}(14)-\mathrm{C}(13)-\mathrm{Yb}(1)-\mathrm{O}(1)$ & $-109.6(2)$ \\
\hline$C(18)-C(13)-Y b(1)-O(1)$ & $15.2(4)$ \\
\hline$C(12)-C(13)-Y b(1)-C(2)$ & $-74.8(3)$ \\
\hline$C(14)-C(13)-Y b(1)-C(2)$ & $39.3(3)$ \\
\hline$C(18)-C(13)-Y b(1)-C(2)$ & $164.2(4)$ \\
\hline$C(12)-C(13)-Y b(1)-C(11)$ & $-36.6(2)$ \\
\hline$C(14)-C(13)-Y b(1)-C(11)$ & $77.5(3)$ \\
\hline$C(18)-C(13)-Y b(1)-C(11)$ & $-157.6(5)$ \\
\hline$C(12)-C(13)-Y b(1)-C(1)$ & $-24.8(5)$ \\
\hline$C(14)-C(13)-Y b(1)-C(1)$ & $89.4(4)$ \\
\hline$C(18)-C(13)-Y b(1)-C(1)$ & $-145.7(4)$ \\
\hline$C(14)-C(13)-Y b(1)-C(12)$ & $114.1(3)$ \\
\hline$C(18)-C(13)-Y b(1)-C(12)$ & $-121.0(5)$ \\
\hline$C(12)-C(13)-Y b(1)-C(15)$ & $-77.0(3)$ \\
\hline$C(14)-C(13)-Y b(1)-C(15)$ & $37.1(2)$ \\
\hline$C(18)-C(13)-Y b(1)-C(15)$ & $162.0(5)$ \\
\hline$C(12)-C(13)-Y b(1)-C(3)$ & $-120.8(3)$ \\
\hline$C(14)-C(13)-Y b(1)-C(3)$ & $-6.7(3)$ \\
\hline$C(18)-C(13)-Y b(1)-C(3)$ & $118.2(4)$ \\
\hline$C(12)-C(13)-Y b(1)-C(5)$ & $115.3(6)$ \\
\hline$C(14)-C(13)-Y b(1)-C(5)$ & $-130.5(6)$ \\
\hline$C(18)-C(13)-Y b(1)-C(5)$ & $-5.6(9)$ \\
\hline$C(12)-C(13)-Y b(1)-C(14)$ & $-114.1(3)$ \\
\hline$C(18)-C(13)-Y b(1)-C(14)$ & $124.9(5)$ \\
\hline
\end{tabular}




\begin{tabular}{|c|c|}
\hline$C(12)-C(13)-Y b(1)-C(4)$ & $-166.8(2)$ \\
\hline $\mathrm{C}(14)-\mathrm{C}(13)-\mathrm{Yb}(1)-\mathrm{C}(4)$ & $-52.6(3)$ \\
\hline$C(18)-C(13)-Y b(1)-C(4)$ & $72.3(5)$ \\
\hline $\mathrm{C}(12)-\mathrm{C}(13)-\mathrm{Yb}(1)-\mathrm{Ga}(1)$ & $47.7(2)$ \\
\hline $\mathrm{C}(14)-\mathrm{C}(13)-\mathrm{Yb}(1)-\mathrm{Ga}(1)$ & $161.9(2)$ \\
\hline $\mathrm{C}(18)-\mathrm{C}(13)-\mathrm{Yb}(1)-\mathrm{Ga}(1)$ & $-73.3(4)$ \\
\hline $\mathrm{C}(21)-\mathrm{Ga}(1)-\mathrm{Yb}(1)-\mathrm{O}(1)$ & $10.0(2)$ \\
\hline $\mathrm{C}(24)-\mathrm{Ga}(1)-\mathrm{Yb}(1)-\mathrm{O}(1)$ & $-142.3(2)$ \\
\hline $\mathrm{C}(25)-\mathrm{Ga}(1)-\mathrm{Yb}(1)-\mathrm{O}(1)$ & $-69.8(3)$ \\
\hline $\mathrm{C}(22)-\mathrm{Ga}(1)-\mathrm{Yb}(1)-\mathrm{O}(1)$ & $85.14(19)$ \\
\hline $\mathrm{C}(23)-\mathrm{Ga}(1)-\mathrm{Yb}(1)-\mathrm{O}(1)$ & $151.50(17)$ \\
\hline $\mathrm{C}(21)-\mathrm{Ga}(1)-\mathrm{Yb}(1)-\mathrm{C}(2)$ & $-123.0(2)$ \\
\hline $\mathrm{C}(24)-\mathrm{Ga}(1)-\mathrm{Yb}(1)-\mathrm{C}(2)$ & $84.7(2)$ \\
\hline $\mathrm{C}(25)-\mathrm{Ga}(1)-\mathrm{Yb}(1)-\mathrm{C}(2)$ & $157.2(3)$ \\
\hline $\mathrm{C}(22)-\mathrm{Ga}(1)-\mathrm{Yb}(1)-\mathrm{C}(2)$ & $-47.90(19)$ \\
\hline $\mathrm{C}(23)-\mathrm{Ga}(1)-\mathrm{Yb}(1)-\mathrm{C}(2)$ & $18.47(18)$ \\
\hline $\mathrm{C}(21)-\mathrm{Ga}(1)-\mathrm{Yb}(1)-\mathrm{C}(11)$ & $139.8(2)$ \\
\hline $\mathrm{C}(24)-\mathrm{Ga}(1)-\mathrm{Yb}(1)-\mathrm{C}(11)$ & $-12.5(2)$ \\
\hline$C(25)-\mathrm{Ga}(1)-\mathrm{Yb}(1)-\mathrm{C}(11)$ & $60.0(3)$ \\
\hline $\mathrm{C}(22)-\mathrm{Ga}(1)-\mathrm{Yb}(1)-\mathrm{C}(11)$ & $-145.1(2)$ \\
\hline $\mathrm{C}(23)-\mathrm{Ga}(1)-\mathrm{Yb}(1)-\mathrm{C}(11)$ & $-78.71(18)$ \\
\hline $\mathrm{C}(21)-\mathrm{Ga}(1)-\mathrm{Yb}(1)-\mathrm{C}(1)$ & $-110.5(2)$ \\
\hline $\mathrm{C}(24)-\mathrm{Ga}(1)-\mathrm{Yb}(1)-\mathrm{C}(1)$ & $97.2(2)$ \\
\hline $\mathrm{C}(25)-\mathrm{Ga}(1)-\mathrm{Yb}(1)-\mathrm{C}(1)$ & $169.7(3)$ \\
\hline $\mathrm{C}(22)-\mathrm{Ga}(1)-\mathrm{Yb}(1)-\mathrm{C}(1)$ & $-35.4(2)$ \\
\hline $\mathrm{C}(23)-\mathrm{Ga}(1)-\mathrm{Yb}(1)-\mathrm{C}(1)$ & $31.00(18)$ \\
\hline $\mathrm{C}(21)-\mathrm{Ga}(1)-\mathrm{Yb}(1)-\mathrm{C}(12)$ & $113.1(2)$ \\
\hline $\mathrm{C}(24)-\mathrm{Ga}(1)-\mathrm{Yb}(1)-\mathrm{C}(12)$ & $-39.2(2)$ \\
\hline $\mathrm{C}(25)-\mathrm{Ga}(1)-\mathrm{Yb}(1)-\mathrm{C}(12)$ & $33.2(3)$ \\
\hline $\mathrm{C}(22)-\mathrm{Ga}(1)-\mathrm{Yb}(1)-\mathrm{C}(12)$ & $-171.8(2)$ \\
\hline $\mathrm{C}(23)-\mathrm{Ga}(1)-\mathrm{Yb}(1)-\mathrm{C}(12)$ & $-105.48(19)$ \\
\hline $\mathrm{C}(21)-\mathrm{Ga}(1)-\mathrm{Yb}(1)-\mathrm{C}(15)$ & $137.6(2)$ \\
\hline $\mathrm{C}(24)-\mathrm{Ga}(1)-\mathrm{Yb}(1)-\mathrm{C}(15)$ & $-14.7(2)$ \\
\hline $\mathrm{C}(25)-\mathrm{Ga}(1)-\mathrm{Yb}(1)-\mathrm{C}(15)$ & $57.8(3)$ \\
\hline $\mathrm{C}(22)-\mathrm{Ga}(1)-\mathrm{Yb}(1)-\mathrm{C}(15)$ & $-147.3(2)$ \\
\hline $\mathrm{C}(23)-\mathrm{Ga}(1)-\mathrm{Yb}(1)-\mathrm{C}(15)$ & $-80.93(19)$ \\
\hline $\mathrm{C}(21)-\mathrm{Ga}(1)-\mathrm{Yb}(1)-\mathrm{C}(3)$ & $-98.7(2)$ \\
\hline $\mathrm{C}(24)-\mathrm{Ga}(1)-\mathrm{Yb}(1)-\mathrm{C}(3)$ & $109.0(2)$ \\
\hline $\mathrm{C}(25)-\mathrm{Ga}(1)-\mathrm{Yb}(1)-\mathrm{C}(3)$ & $-178.6(3)$ \\
\hline $\mathrm{C}(22)-\mathrm{Ga}(1)-\mathrm{Yb}(1)-\mathrm{C}(3)$ & $-23.6(2)$ \\
\hline $\mathrm{C}(23)-\mathrm{Ga}(1)-\mathrm{Yb}(1)-\mathrm{C}(3)$ & $42.73(19)$ \\
\hline $\mathrm{C}(21)-\mathrm{Ga}(1)-\mathrm{Yb}(1)-\mathrm{C}(5)$ & $-79.7(2)$ \\
\hline $\mathrm{C}(24)-\mathrm{Ga}(1)-\mathrm{Yb}(1)-\mathrm{C}(5)$ & $128.0(2)$ \\
\hline $\mathrm{C}(25)-\mathrm{Ga}(1)-\mathrm{Yb}(1)-\mathrm{C}(5)$ & $-159.5(3)$ \\
\hline $\mathrm{C}(22)-\mathrm{Ga}(1)-\mathrm{Yb}(1)-\mathrm{C}(5)$ & $-4.62(19)$ \\
\hline $\mathrm{C}(23)-\mathrm{Ga}(1)-\mathrm{Yb}(1)-\mathrm{C}(5)$ & $61.74(18)$ \\
\hline $\mathrm{C}(21)-\mathrm{Ga}(1)-\mathrm{Yb}(1)-\mathrm{C}(14)$ & $101.9(2)$ \\
\hline $\mathrm{C}(24)-\mathrm{Ga}(1)-\mathrm{Yb}(1)-\mathrm{C}(14)$ & $-50.4(2)$ \\
\hline $\mathrm{C}(25)-\mathrm{Ga}(1)-\mathrm{Yb}(1)-\mathrm{C}(14)$ & $22.1(3)$ \\
\hline $\mathrm{C}(22)-\mathrm{Ga}(1)-\mathrm{Yb}(1)-\mathrm{C}(14)$ & $177.0(2)$ \\
\hline $\mathrm{C}(23)-\mathrm{Ga}(1)-\mathrm{Yb}(1)-\mathrm{C}(14)$ & $-116.7(2)$ \\
\hline $\mathrm{C}(21)-\mathrm{Ga}(1)-\mathrm{Yb}(1)-\mathrm{C}(4)$ & $-71.4(2)$ \\
\hline $\mathrm{C}(24)-\mathrm{Ga}(1)-\mathrm{Yb}(1)-\mathrm{C}(4)$ & $136.3(2)$ \\
\hline $\mathrm{C}(25)-\mathrm{Ga}(1)-\mathrm{Yb}(1)-\mathrm{C}(4)$ & $-151.3(3)$ \\
\hline $\mathrm{C}(22)-\mathrm{Ga}(1)-\mathrm{Yb}(1)-\mathrm{C}(4)$ & $3.7(2)$ \\
\hline $\mathrm{C}(23)-\mathrm{Ga}(1)-\mathrm{Yb}(1)-\mathrm{C}(4)$ & $70.04(18)$ \\
\hline $\mathrm{C}(21)-\mathrm{Ga}(1)-\mathrm{Yb}(1)-\mathrm{C}(13)$ & $90.9(2)$ \\
\hline $\mathrm{C}(24)-\mathrm{Ga}(1)-\mathrm{Yb}(1)-\mathrm{C}(13)$ & $-61.4(2)$ \\
\hline
\end{tabular}




$$
\begin{aligned}
& \mathrm{C}(25)-\mathrm{Ga}(1)-\mathrm{Yb}(1)-\mathrm{C}(13) \\
& \mathrm{C}(22)-\mathrm{Ga}(1)-\mathrm{Yb}(1)-\mathrm{C}(13) \\
& \mathrm{C}(23)-\mathrm{Ga}(1)-\mathrm{Yb}(1)-\mathrm{C}(13)
\end{aligned}
$$

$11.1(3)$

$166.02(19)$

$-127.61(18)$

Symmetry transformations used to generate equivalent atoms: 
